\title{
Multimorbidity, Management of COPD and Health Outcomes among Medicaid Beneficiaries
}

\author{
Mayank Ajmera
}

Follow this and additional works at: https://researchrepository.wvu.edu/etd

\section{Recommended Citation}

Ajmera, Mayank, "Multimorbidity, Management of COPD and Health Outcomes among Medicaid Beneficiaries" (2014). Graduate Theses, Dissertations, and Problem Reports. 5039.

https://researchrepository.wvu.edu/etd/5039

This Dissertation is protected by copyright and/or related rights. It has been brought to you by the The Research Repository @ WVU with permission from the rights-holder(s). You are free to use this Dissertation in any way that is permitted by the copyright and related rights legislation that applies to your use. For other uses you must obtain permission from the rights-holder(s) directly, unless additional rights are indicated by a Creative Commons license in the record and/ or on the work itself. This Dissertation has been accepted for inclusion in WVU Graduate Theses, Dissertations, and Problem Reports collection by an authorized administrator of The Research Repository @ WVU.

For more information, please contact researchrepository@mail.wvu.edu. 
Multimorbidity, Management of COPD and Health Outcomes among Medicaid Beneficiaries

Mayank Ajmera

\author{
Dissertation submitted \\ to the School of Pharmacy \\ at the West Virginia University \\ in partial fulfillment of the requirements for the degree of \\ Doctor of Philosophy \\ in \\ Health Outcomes Research
}

Usha Sambamoorthi, Ph.D., Chair

Cindy Tworek, Ph.D.

Nilanjana Dwibedi, Ph.D.

Aaron Metzger, Ph.D.

George Rust, M.D, M.P.H.

This study was partially supported by a grant from the Agency for Healthcare Research and Quality (AHRQ):1R24 HS018622-01

Department of Pharmaceutical Systems and Policy

Morgantown, West Virginia

2014

Keywords: Chronic Obstructive Pulmonary Disease, Multimorbidity, Management, Novel Therapies, New-Onset Diabetes

Copyright 2014 Mayank Ajmera 


\section{ABSTRACT \\ Multimorbidity, Management of COPD and Health Outcomes among Medicaid Beneficiaries}

Mayank Ajmera, B.Pharm., M.S.

Chronic Obstructive Pulmonary Disease (COPD) is characterized by persistent and progressive airflow limitation caused due to chronic inflammation in the lungs. Approximately 15 million adults in the United States (U.S.) are estimated to be diagnosed with COPD and an equal number may have undiagnosed COPD. Challenges to COPD management include high prevalence of inflammationrelated multimorbidity among individuals with COPD. The association between multimorbidity, existing COPD management and role of novel therapies with anti-inflammatory properties (e.g. statins) in improving COPD-specific outcomes is not well researched. Therefore, the purpose of this study was to use real-world observational data to provide a comprehensive understanding of the relationship between multimorbidity and COPD management as well as assess the effectiveness and safety of statins in terms of COPD management. The specific aims of three studies were to: (1) examine the association between inflammation-related multimorbidity and COPD management in terms of COPD medication receipt, long-acting bronchodilators persistence and COPD-specific outcomes; (2) assess the effectiveness of novel statin therapy in improving COPD-specific outcomes; (3) evaluate the safety of statins and other commonly used medications (antidepressants and inhaled corticosteroids) in terms of new-onset diabetes. This study used a retrospective longitudinal dynamic cohort design using data extracted from multiple years (2005-2008) of Medicaid Analytic eXtract (MAX) files to identify Medicaid beneficiaries with newly diagnosed COPD $(n=19,060)$. Findings from the first study documented very high prevalence of inflammation-related multimorbidity and indicated that it was significantly associated with reduced COPD-medication utilization and decreased persistence on long-bronchodilators. Our study findings suggest that COPD medication management may be poor due to competing demands arising from the presence of inflammation-related multimorbidity. The results from the study on effectiveness of statins revealed that any statin use improved COPD-specific outcomes compared to no statin use. A closer examination of the data revealed that only those with long-term statin use had better outcomes as compared to those with no statin use. We also found that beneficiaries with inflammation-related multimorbidity and statin use had better COPD-specific outcomes compared to those with multimorbidity and no statin use. From the third study, we found that association between statin use and risk of new-onset diabetes was no longer significant in analyses that controlled for selection bias in unobserved characteristics. Collectively, these findings indicate poor COPD management among those with multimorbidity and emphasize the need for novel therapies to effectively manage COPD. In this context, the current study underscores the advantage of statins in improving COPD-specific clinical and economic outcomes. This study indicate the need of randomized clinical trials and longterm observational studies to establish the efficacy, effectiveness, and safety of novel therapeutic agents in management of COPD. 


\section{DEDICATION}

I dedicate this dissertation to my spiritual guru, P.P. Pramukh Swami Maharaj, my late grandfather, Mr. Hargovinddas Ajmera, my grandmother, Mrs. Leelaben Ajmera, my mom, Mrs. Asha Ajmera, my dad, Mr. Rakesh Ajmera, my brother, Dev and my wife, Amruta without whose love and support this would not have been possible. 


\section{ACKNOWLEDGEMENTS}

I am grateful to several individuals who have tremendously contributed in all the research endeavors during my Ph.D. at WVU as well as shared invaluable life experiences at all stages of project work. First, I would like to thank my mentor Dr. Usha Sambamoorthi for being an amazing mentor and most importantly for constantly believing in me throughout my graduate studies.

Without her patience and consistent guidance, this dissertation project would not have been possible. She has always there to help me and strengthen me during times of crisis. Her contribution in shaping my research and career paths has been priceless. I am filled with joy and pride to attribute my doctoral education to tremendous training provided by Dr. Sambamoorthi.

I would also like to thank my committee members Drs. Tworek, Pan, Dwibedi, Metzger and Rust for their critical input and insight in my dissertation work. A special thanks goes to Dr. Rust for helping me in understanding the clinical aspects of the study. I would also like to acknowledge the WVU family: Drs. Scott, Pan, Kelly, Angie Frame, and all the graduate student friends for making my time at WVU exceptionally memorable. I would also like to extend my heartfelt gratitude to Dr. Madhavan who has always been like a father figure for me throughout my time at WVU.

I would like to thank the Centers for Medicare and Medicaid Services (CMS) and Research and Data Assistance Center (ResDAC) providing the research files for Medicaid beneficiaries.

I am eternally grateful for all the wonderful people in my life without whose love and support I would not be the same person that I am today. I have been guided by my spiritual guru P.P. Pramukh Swami Maharaj, whose teachings have inspired me to achieve this milestone and never give up on my goals. This academic achievement was solely due to Swamishri's blessings. I would like to take this opportunity to remember my late grandfather 'Dadaji', whose simplicity and enthusiasm for life have shaped my personality and helped me in achieving this feat. I am thankful to my grandmother 'Ba', for the love and affection she has showered upon me and molded me into the individual I am today. I would like to specially express my love and gratitude to my mom and dad for their unwavering support and encouragement throughout this journey. Special thanks to my wonderful family including my brothers 'Dev' and 'Siddharth Bhai', my uncle ('bapuji') and aunt ('bhabha'), and Meghna bhabhi, who have always lent an ear and shoulder to lean on during this critical time. I am grateful to my in-laws and my sister-in-law, Aditi who have always inspired me to achieve my goals and strive for excellence. Finally, yet most importantly, I am indebted to my wife, Amruta, for her moral support, love and belief in me, who was always constantly motivating me, providing strength and standing by me in my good and hard times. 


\section{TABLE OF CONTENTS}

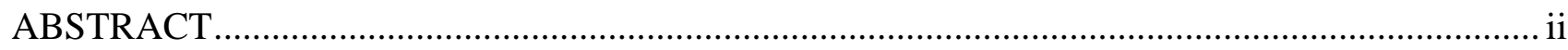

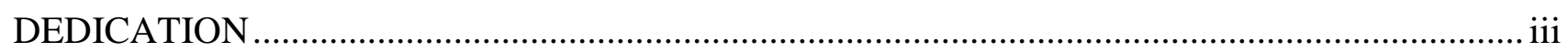

ACKNOWLEDGEMENTS ................................................................................................. iv

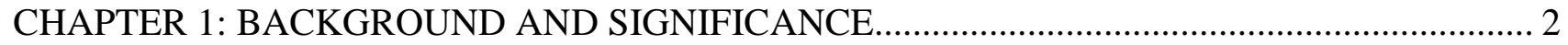

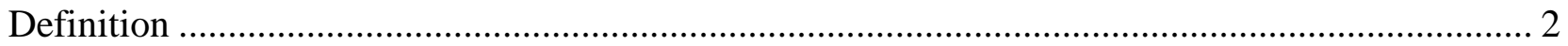

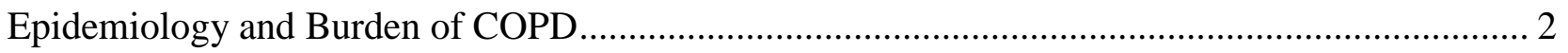

Inflammation-related Multimorbidity and COPD-specific Outcomes............................................ 3

Inflammation-related Multimorbidity, Novel Statin Therapy and COPD-specific outcomes .......... 4

Inflammation-related Multimorbidity, Medication Use and New-onset Diabetes............................ 5

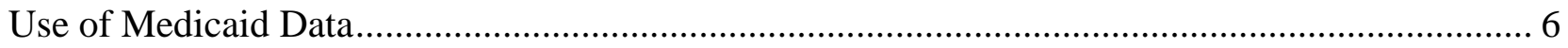

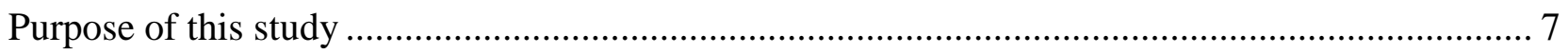

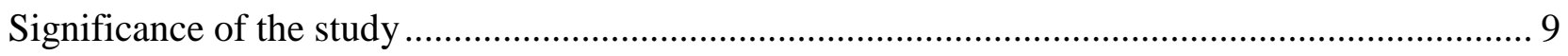

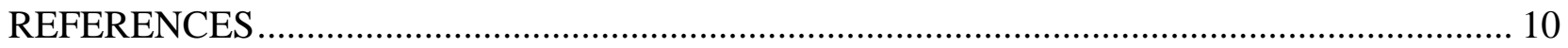

CHAPTER 2: INFLAMMATION-RELATED MULTIMORBIDITY AND COPD MANAGEMENT AMONG MEDICAID BENEFICIARIES WITH NEWLY-DIAGNOSED COPD........................... 14

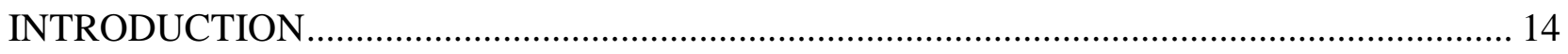

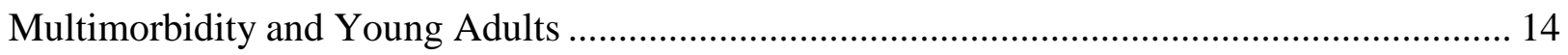

Multimorbidity among Adults with Chronic Obstructive Pulmonary Disease (COPD) ............. 16

Prevalence of Inflammation-related multimorbidity among individuals with COPD................. 17

Challenges to COPD Management...................................................................................... 18

Impact on Overall Health Outcomes ................................................................................ 19

Effects on COPD-Specific Outcomes............................................................................. 19

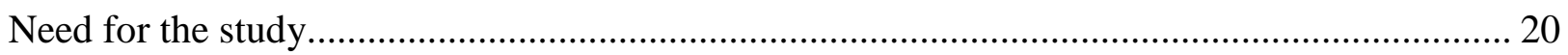

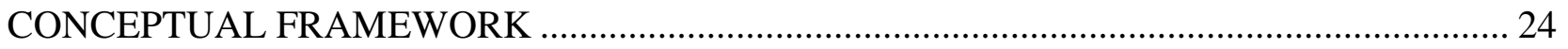

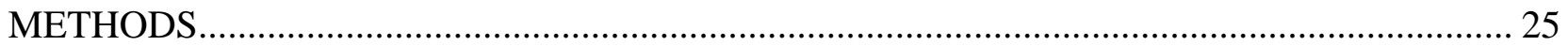

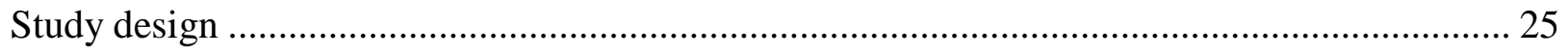

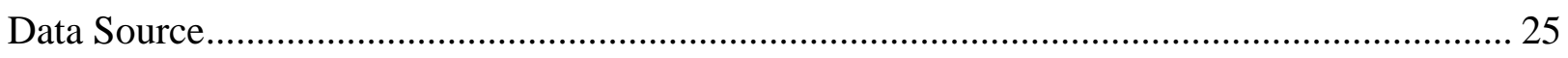

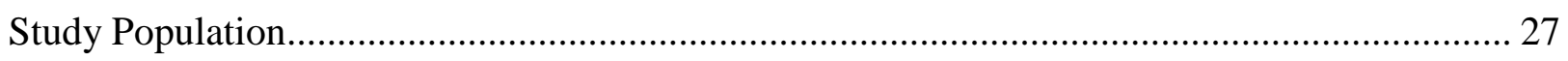

Dependent Variables..................................................................................................... 28

Key Independent Variable: Inflammation-related Multimorbidity ……….................................. 30

Other Independent Variables ................................................................................................ 31 


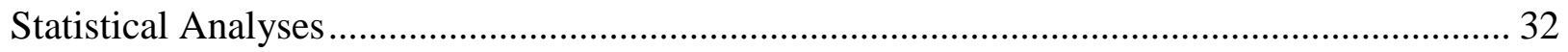

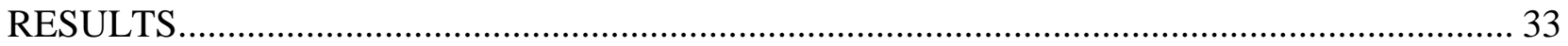

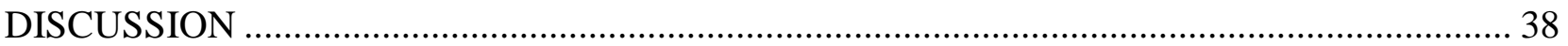

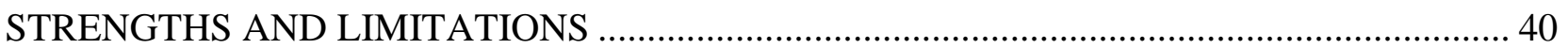

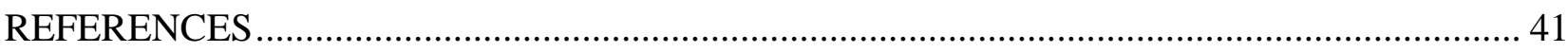

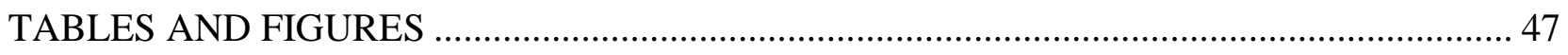

Figure: Algorithm Describing Selection of Study Population.................................................... 47

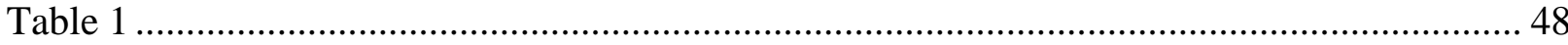

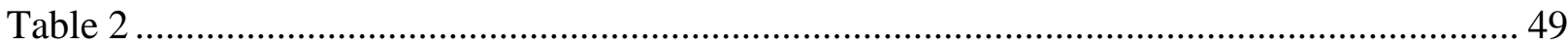

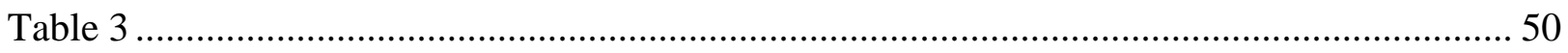

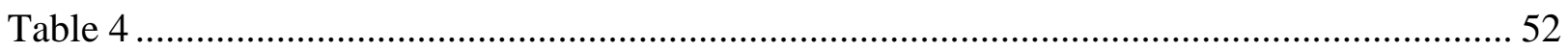

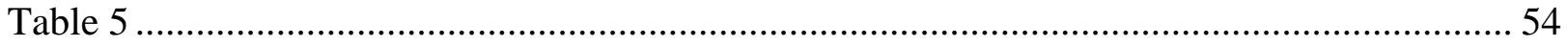

CHAPTER 3: REAL-WORLD STUDY OF CLINICAL AND ECONOMIC OUTCOMES OF STATIN MEDICATIONS AMONG MEDICAID BENEFICIARIES WITH NEWLY-DIAGNOSED

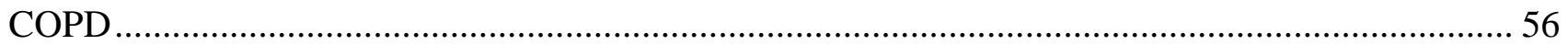

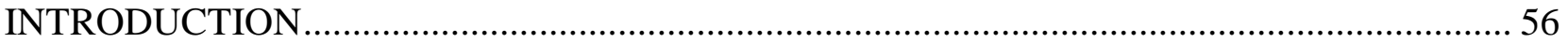

Inability of Current Medications to Treat Systemic Inflammation …………………………......5 57

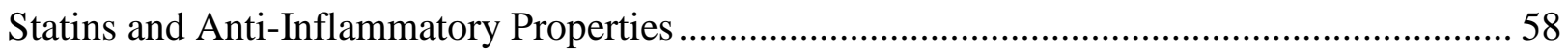

Statin use and Improved Outcomes among individuals with COPD........................................... 58

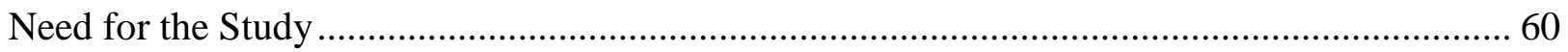

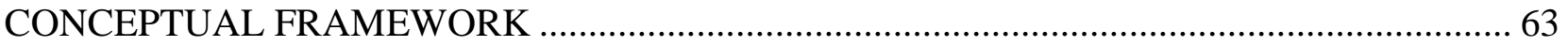

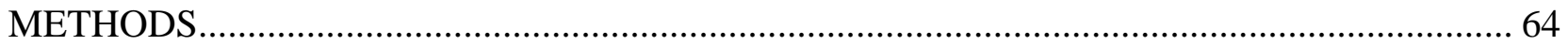

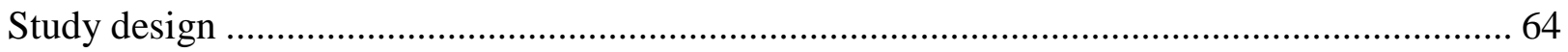

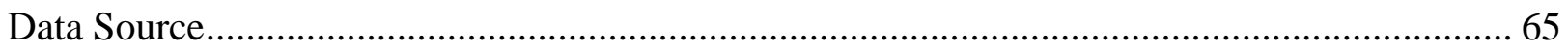

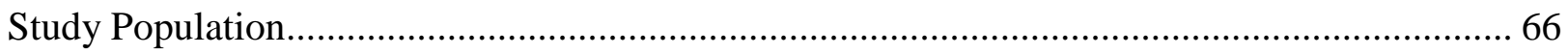

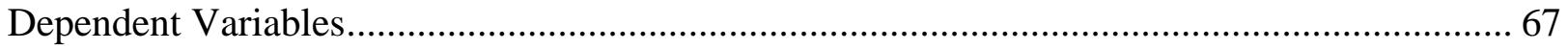

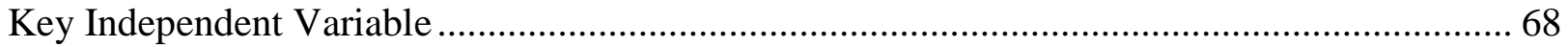

Other Independent Variables (External Controls) …………................................................... 70

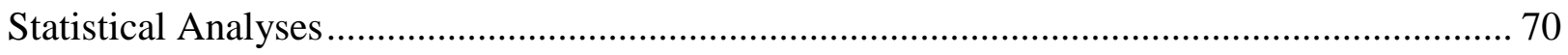

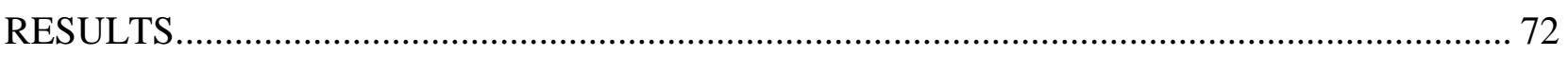

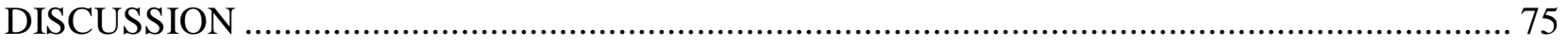

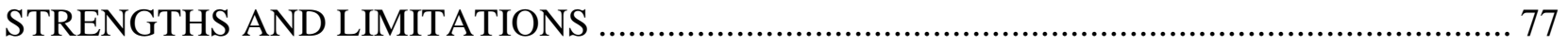

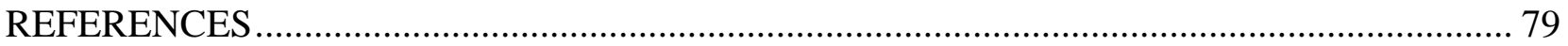




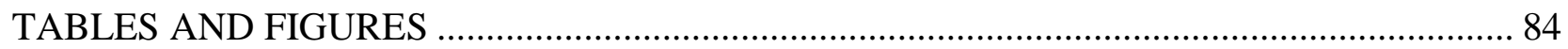

Figure: Algorithm Describing Selection of Study Population................................................ 84

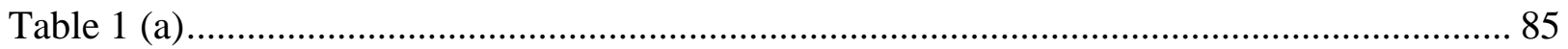

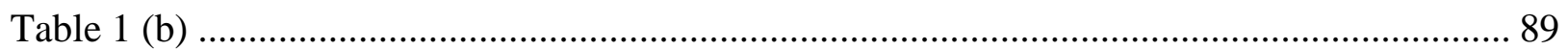

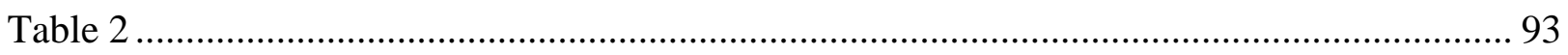

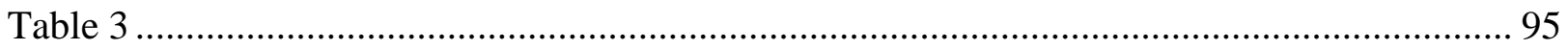

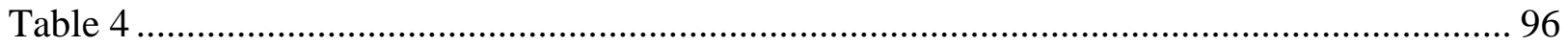

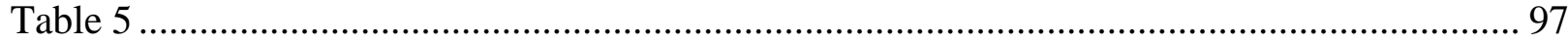

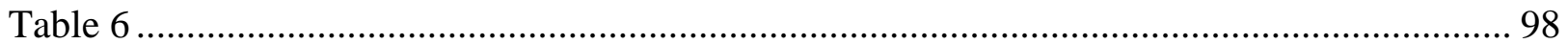

CHAPTER 4: ANTIDEPRESSANTS, INHALED CORTICOSTEROIDS, STATINS AND NEWONSET DIABETES AMONG MEDICAID BENEFICIARIES WITH NEWLY-DIAGNOSED

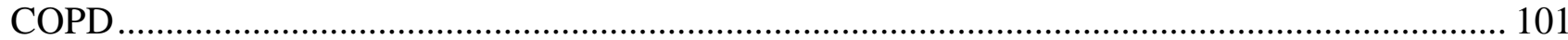

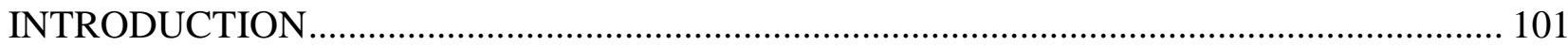

General Biological Correlates of Medications and New-Onset Diabetes ............................. 102

Medication Use and New-onset Diabetes: Existing Evidence .......................................... 103

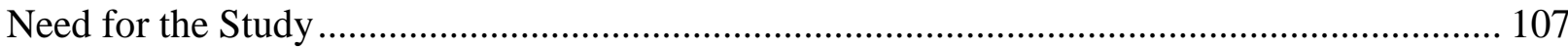

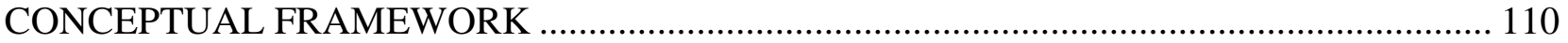

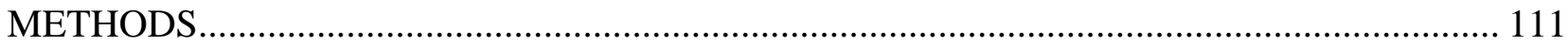

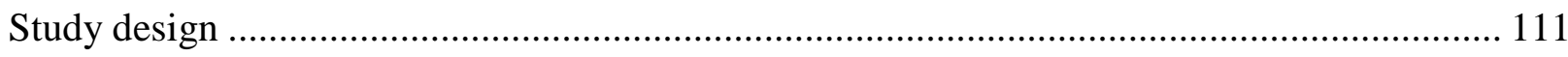

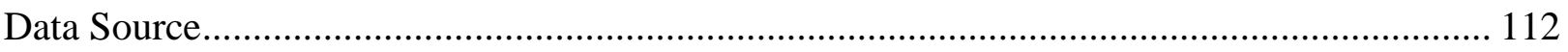

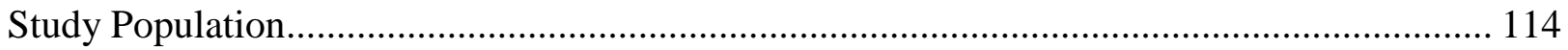

Dependent Variable (New-onset diabetes) ................................................................... 115

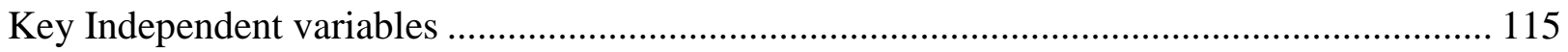

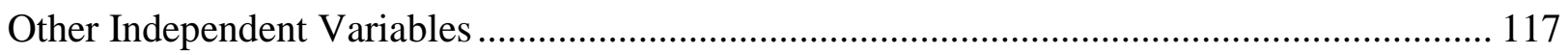

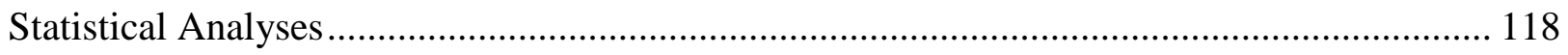

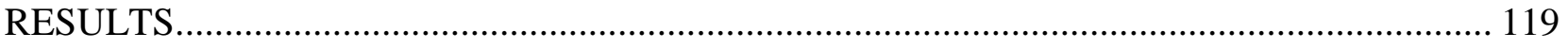

Secondary Analysis (Controlling for Selection Bias in Statin Use) .................................... 121

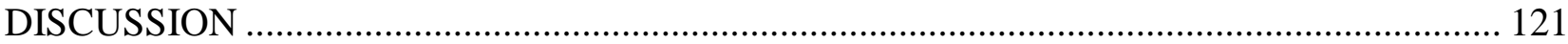

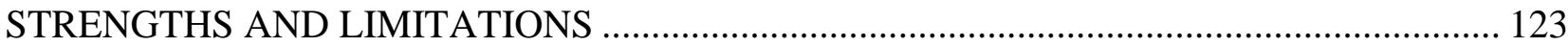

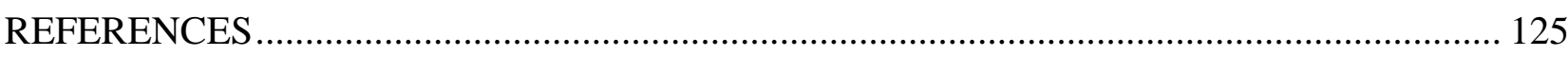

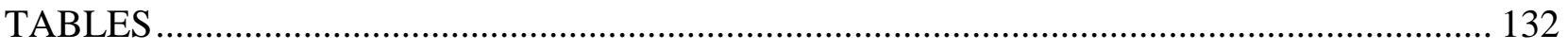

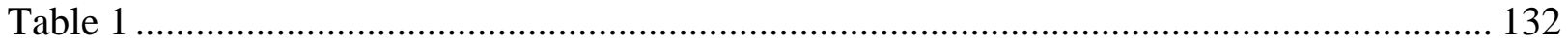

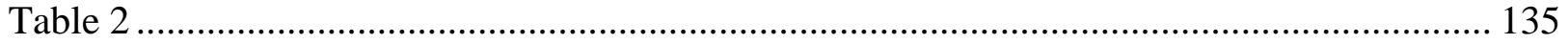




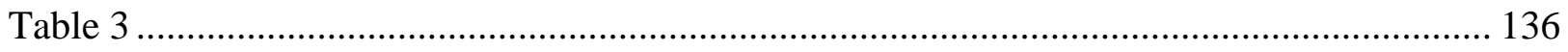

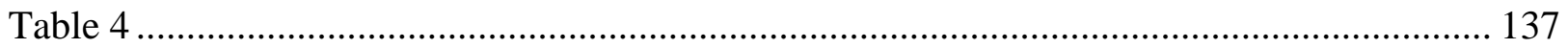

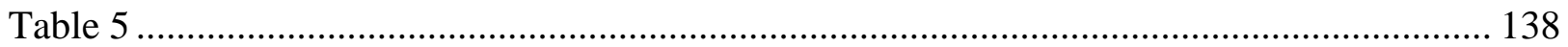

CHAPTER 5: SUMMARY AND CONCLUSIONS ............................................................ 140

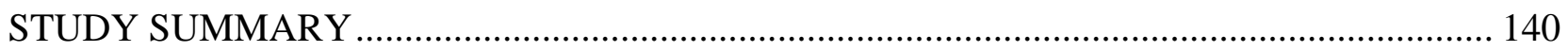

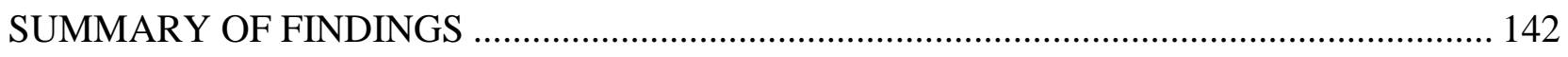

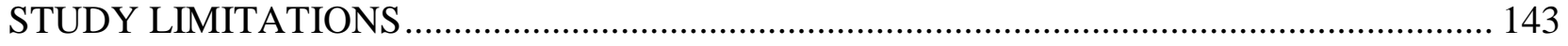

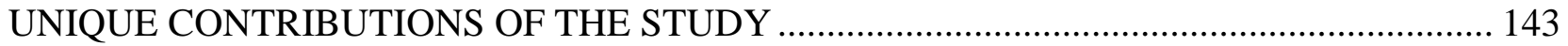

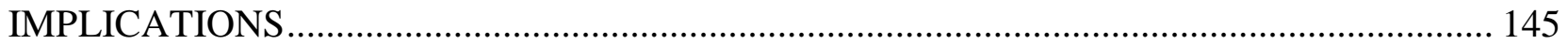

DIRECTIONS FOR FUTURE RESEARCH ............................................................... 146

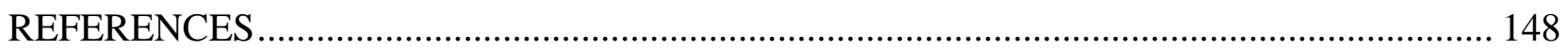


CHAPTER 1 


\section{CHAPTER 1: BACKGROUND AND SIGNIFICANCE}

\section{Definition}

Chronic Obstructive Pulmonary Disease (COPD) is characterized by persistent and progressive airflow limitation caused due to chronic inflammation in the lungs. The inflammation of airways in the lungs is usually a result of persistent exposure to noxious substances such as tobacco smoke. (GOLD, 2013) As per the Global Initiative for Chronic Obstructive Lung Disease (GOLD) definition of COPD, airflow limitation can be mainly attributed to small airways diseases (obstructive bronchiolitis) and parenchymal destruction (emphysema). (GOLD, 2013) The most common symptoms of COPD include dyspnea, chronic cough, sputum production, wheezing and chest tightness. (Kessler, 2011; Espinosa, 2011)

\section{Epidemiology and Burden of COPD}

Approximately 15 million adults in the United States (U.S.) are estimated to be diagnosed with COPD and an equal number may have undiagnosed COPD. (CDC, 2012; Ford et al., 2013) Specifically, COPD is highly prevalent among young adults ( $<65$ years old) as well. The extent of COPD among young adults ranges from 3.2\% (18-44 year) to 9.2\% (55-64 year). (CDC, 2012) COPD is associated with high clinical, economic and humanistic burden. It is the third leading cause of death $(133,965$ deaths due to COPD in 2009) in the U.S. with age adjusted death rate of 41.2 per 100,000 individuals. (NHLBI, 2009) The overall management of COPD accounts for \$49.5 billion, with maximum healthcare expenditures attributed towards exacerbation management. Healthcare utilization, specifically inpatient healthcare utilization among COPD has increased over the years.(Brown et al, 2010) Furthermore, COPD has high humanistic or social burden as well.

Globally, COPD account for $3.1 \%$ of disability adjusted life-years (DALYs) lost; and is projected to 
be the seventh leading cause of DALYs lost. (GOLD, 2013) In addition to the burden associated with COPD, those with COPD also have high rates of multimorbidity; specifically inflammationrelated multimorbidity. The high prevalence of inflammation-related multimorbidity adds to the burden of COPD. In this context, it is important to systematically study the relationship between inflammation-related multimorbidity, management of COPD and health outcomes.

\section{Inflammation-related Multimorbidity and COPD-specific Outcomes}

COPD is mainly characterized as an inflammatory disease (Rabe et al., 2007) and therefore, it has been suggested that individuals with COPD may have high prevalence of other inflammationrelated chronic conditions. Investigators from a study using National Health and Nutrition Examination Survey (NHANES) have reported that individuals with COPD have high rates of inflammation-related chronic diseases such as (54.6\% vs. $36.9 \%)$, diabetes $(16.3 \%$ vs. $12.8 \%)$, depression (20.6\% vs. $12.5 \%$ ), osteoporosis (16.9\% vs. $8.5 \%)$, coronary heart disease (12.7\% vs. $6.1 \%)$, congestive heart failure (12.1\% vs. $3.9 \%)$, and stroke (8.9\% vs. $4.6 \%)$ as compared to those without COPD. (Schnell et al., 2012) Indeed, researchers have suggested that COPD should be deemed as part of "chronic systemic inflammatory syndrome”. (Barnes \& Celli, 2009; Fabbri, 2008) Evidence exists with regards to challenges to COPD management that may occur due to presence of inflammation-related multimorbidity. However, no study till now has assessed its impact on COPD management, specifically treatment patterns among individuals with COPD. Furthermore, only a few studies have examined the role of co-existing chronic conditions on COPD-specific outcomes. The findings from these studies point towards negative impact of multimorbidity on COPD-specific outcomes; however, none of the studies until now have systematically evaluated the impact of inflammation-related multimorbidity on COPD-specific outcomes. 
This leads to the rationale for the Aim1, which examines the association between inflammation-related multimorbidity and COPD treatment patterns (management) and COPDspecific outcomes such as COPD-specific healthcare utilization and healthcare expenditures among Medicaid beneficiaries with newly-diagnosed COPD.

\section{Inflammation-related Multimorbidity, Novel Statin Therapy and COPD-specific outcomes}

Currently, the treatment for Chronic Obstructive Pulmonary Disease (COPD) focuses on reducing the frequency of acute exacerbations and relieving the symptoms of COPD such as shortness of breath (GOLD, 2013) through acute and maintenance pharmacologic therapies as well as pulmonary rehabilitation. To date, no therapeutic options, which alter the prognosis of disease also known as disease modifying drugs for COPD treatment, have been established. As the literature review from Aim 1 suggested that COPD is associated with high levels of systemic inflammation; it is plausible that treatments for reducing the systemic inflammation may have positive COPDspecific outcomes. Indeed, new insights into the pathophysiology of the disease have opened up opportunities to use therapeutic regimens that are currently available for circulatory diseases to treat COPD. (M. Cazzola, Ciaprini, Page, \& Matera, 2007; Fabbri \& Rabe, 2007)

Statins, a class of cholesterol lowering drugs have demonstrated their effectiveness in prevention and management of cardiovascular diseases. (Baigent et al., 2005) Based on the review of in vitro as well as in vivo studies Schönbeck and Libby (Schonbeck \& Libby, 2004) concluded that statins also have anti-inflammatory functions. The evidence has also been supported by preclinical models that have revealed the pleiotropic/anti-inflammatory therapeutic benefits attributable to statins. (Takahashi et al., 2008) These facts derived from several mechanistic studies suggest that statin use has emerged as a novel therapeutic intervention for individuals with COPD. (Walsh, 2008) 
Some studies have examined the beneficial effects of statins in improving lung function, health status and COPD-related exacerbations among those with COPD. Few studies have assessed impact of statin use and COPD-specific outcomes and reported lower rates of COPD-related hospitalizations with statin use. However, none of the studies are generalizable to US population and no study has systematically studied the role of inflammation-related multimorbidity on statin use and COPD-specific outcomes. Moreover, no study till now has examined the impact of statin use on healthcare expenditures among individuals with COPD.

Therefore, the primary purpose of the Aim 2, is to examine the effectiveness of novel statin therapy in terms of clinical and economic outcomes among individuals with newly-diagnosed COPD in a real-world practice setting. The study estimates the extent of statin use among individuals with COPD and examined the factors associated with statin use. Furthermore, we also assessed the COPD-specific outcomes with statin use among those with COPD and inflammation-related multimorbidity.

\section{Inflammation-related Multimorbidity, Medication Use and New-onset Diabetes}

Multiple medication use among individuals with COPD is very high due to the presence of high rates of inflammation-related multimorbidity. (Anecchino et al., 2007) Although, generally regarded as safe, concerns about adverse effects have been raised about some of the medications used to treat COPD and allied inflammation-related multimorbidities. Specifically, antidepressants, inhaled corticosteroids (ICS) and statins have been linked with new-onset diabetes. (Bhattacharjee, Bhattacharya, Kelley, \& Sambamoorthi, 2013; O'Byrne et al., 2012; Sattar et al., 2010; Suissa, Kezouh, \& Ernst, 2010) New-onset diabetes has been linked with medication use because some of these medications can increase insulin resistance and decrease insulin secretion and thereby affecting the overall glucose metabolism. (Carvalho et al., 2004; Colbert \& Stone, 2012; Nielsen et al., 2004) 
Medications such as ICS are integral to the management of individuals with COPD. Furthermore, new medications (statins) are being evaluated as disease modifying drugs for the treatment of individuals with COPD. Therefore, it is essential to generate evidence regarding multiple medication use and potential adverse effects like new-onset diabetes.

Thus, in the Aim 3, we comprehensively evaluated the association between commonly-used medications and new-onset diabetes among individuals with COPD and inflammation-related multimorbidity. This study specifically examined the safety of type of statins among Medicaid beneficiaries with COPD after controlling for comprehensive set of risk factors.

\section{Use of Medicaid Data}

To accomplish all the aims we selected Medicaid data for the following reasons: 1) Multimorbidity rates are increasing among the young adults and studies have reported negative impact of multimorbidity on economic outcomes among the young adults population. In the United States, Medicaid is the largest insurance provider for the young adults and thus it is important to examine the impact of multimorbidity on Medicaid enrollees. 2) Furthermore, Affordable Care Act through the Medicaid expansion policies is going to expand the coverage for young adults and thus will include a majority of young adults in the US. 3) Medicaid population also represents individuals in the lower socio-economic strata of the society. The effects of socio-economic status on lung functions have been extensively studied. It has been reported that low socio-economic status (SES) is associated with decreased lung function even after controlling for smoking status, occupational exposures and race. A review of literature by Hegewald et al. concluded that SES is an underrecognized factor of pulmonary disease. (Hegewald, 2007) Many factors associated with low SES such as exposure to indoor and outdoor air pollutants, poor nutrition, tobacco smoke exposure etc. have been suggested to affect lung function. (Hegewald, 2007) Therefore, understanding the 
management of COPD in a more vulnerable section of the society with greater risk factors is important. 4) Specifically, COPD management in Medicaid enrollees has been an understudied area. Few studies have examined healthcare utilization, economic burden and quality of care among Medicaid beneficiaries with COPD. The burden of COPD in high-risk Medicaid population (40-64 years old) is greater when compared with other respiratory diseases (ORD) and non-respiratory diseases (NRD). (Shaya, 2006; Lin, 2010) In a study conducted by Shaya FT et al, it has been suggested that healthcare use i.e. hospitalization rates are more among COPD patients as compared to ORD and NRD patients within the Medicaid population. In the same study, it has been reported the office visits (indicator of better quality) were lower among COPD patients as compared to ORD patients. (Shaya, 2006) Studies have also shown that Medicaid beneficiaries with COPD have high rates of multimorbidity. Therefore, it is important to systematically study the management of COPD in the Medicaid population. (Lin, 2010)

\section{Purpose of this study}

The purpose of this study was to comprehensively understand the relationship between inflammation-related multimorbidity, COPD management and health outcomes associated with novel statin therapy among Medicaid beneficiaries observed in a real-world practice setting. This study used a retrospective longitudinal dynamic cohort design using data extracted from multiple years (2005-2008) of Medicaid Analytic eXtract (MAX) files to identify Medicaid beneficiaries with newly diagnosed COPD $(\mathrm{n}=19,060)$. The specific aims and objectives of three studies are listed below.

\section{Aim 1: Examine the association between inflammation-related multimorbidity and COPD medication receipt, long-acting bronchodilators persistence and COPD-specific outcomes among Medicaid beneficiaries with COPD}

Objective 1.1: To examine COPD Medication receipt and long-acting bronchodilators persistence among Medicaid beneficiaries with COPD and inflammation-related multimorbidity defined by the 
presence of arthritis, CVD, depression, diabetes and osteoporosis and depression compared those with COPD only.

Hypotheses 1.1: We hypothesized that individuals with COPD and inflammation-related multimorbidity will have significantly lower likelihood of receiving inhaled medications and will also have lower rates of treatment persistence compared to those with COPD only.

Objective 1.2: Compare COPD-specific (COPD-specific hospitalizations, COPD-specific Emergency Room visits and COPD-specific outpatient visits) and COPD-related healthcare utilization (COPD-related hospitalizations and COPD-related Emergency Room visits) among individuals with COPD and inflammation-related multimorbidity and those with COPD only.

Hypotheses 1.2: We hypothesized that individuals with COPD and inflammation-related multimorbidity will have significantly higher healthcare utilization as compared to individuals with COPD only.

Objective 1.3: Estimate and compare COPD-specific healthcare expenditures individuals with COPD and inflammation-related multimorbidity and those with COPD only.

Hypotheses 1.3: We hypothesized that individuals with COPD and inflammation-related multimorbidity will have significantly higher COPD-specific healthcare expenditures as compared to individuals with COPD only.

\section{Aim 2: To examine the effectiveness of novel statin therapy in improving COPD-specific outcomes.}

Objective 2.1: To estimate the prevalence of statin therapy and examine the factors associated with statin therapy among individuals with COPD.

Objective 2.2: To assess the effectiveness of statin therapy among Medicaid beneficiaries with COPD.

Hypotheses 2.2.1: We hypothesize that adults with statin therapy will be less likely to have COPDspecific hospitalizations, COPD-specific Emergency Room visits, and COPD-specific outpatient visits.

Hypotheses 2.2.2: We hypothesize that adults with statin therapy will be less likely to have COPDspecific hospitalizations, COPD-specific Emergency Room visits, and COPD-specific outpatient visits among individuals with COPD and inflammation-related multimorbidity.

Objective 2.3: To estimate and compare total healthcare and COPD-specific expenditures associated with statin therapy among individuals with COPD compared to no statin therapy.

Hypotheses 2.3: We hypothesize statin therapy will be associated with statistically significant lower total healthcare expenditures and COPD-specific expenditures as compared to adults with no statin therapy. 
Aim 3: To evaluate the association between commonly used medications (antidepressants, ICS, statins) and new-onset diabetes among adults with newly diagnosed COPD.

Objective 3.1: To assess real world safety (new-onset diabetes) of any antidepressants, ICS and statin use among Medicaid beneficiaries with newly-diagnosed COPD after controlling for comprehensive set of risk factors.

Hypotheses 31: We hypothesize that any antidepressants, ICS and statin use will increase the risk of new-onset diabetes among Medicaid beneficiaries with newly-diagnosed COPD compared to those with no antidepressants, no ICS and no statin use.

Objective 3.2: To compare the safety (new-onset diabetes) of type of statins among Medicaid beneficiaries with newly-diagnosed COPD after controlling for comprehensive set of risk factors.

Hypotheses 3.2: We hypothesize that lipophilic statins i.e. atorvastatin and simvastatin will be associated with higher risk of new-onset diabetes compared to non-lipophilic statins such as pravastatin.

Objective 3.3: To examine the association between long-term use of antidepressants, statins and new-onset diabetes among Medicaid beneficiaries with newly-diagnosed COPD after controlling for comprehensive set of risk factors.

Hypotheses 3.3: We hypothesize that long-term use of antidepressants and statins throughout the baseline period will be associated with increased likelihood of incident diabetes as compared to non-persistent use of statins.

\section{Significance of the study}

This study aims to fill the knowledge gap and provide knowledge about real-world patterns of current pharmacologic management among those with inflammation-related multimorbidity and COPD. As there has been an increasing recognition for organizing care around the individuals rather than the disease (Kadam, 2012) an understanding of how care is delivered among individuals with multimorbidity will have important implications for the overall healthcare management of individuals with COPD. Our study also addresses the need to evaluate novel anti-inflammatory treatments for effective management of patients with COPD. As COPD is being increasingly recognized as a disease of systemic inflammation, it is highly important to measure the effectiveness of anti-inflammatory therapies in a real-world setting. 


\section{REFERENCES}

Anecchino, C., Rossi, E., Fanizza, C., De Rosa, M., Tognoni, G., \& Romero, M. (2007). Prevalence of chronic obstructive pulmonary disease and pattern of comorbidities in a general population. International Journal of Chronic Obstructive Pulmonary Disease, 2(4), 567-574.

Baigent, C., Keech, A., Kearney, P. M., Blackwell, L., Buck, G., Pollicino, C., Simes, R. (2005). Efficacy and safety of cholesterol-lowering treatment: Prospective meta-analysis of data from 90,056 participants in 14 randomised trials of statins. Lancet, 366(9493), 1267-1278.

Barnes, P. J., \& Celli, B. R. (2009). Systemic manifestations and comorbidities of COPD. The European Respiratory Journal, 33(5), 1165-1185. doi:10.1183/09031936.00128008

Bhattacharjee, S., Bhattacharya, R., Kelley, G. A., \& Sambamoorthi, U. (2013). Antidepressant use and new-onset diabetes: A systematic review and meta-analysis. Diabetes/metabolism Research and Reviews, 29(4), 273-284. doi:10.1002/dmrr.2393

Bhattacharya R, Ajmera M, Bhattacharjee S, Sambamoorthi U. Use of antidepressants and statins and short-term risk of new-onset diabetes among high risk adults. Diabetes Res Clin Pract. 2014 Jun 4. pii: S0168-8227(14)00199-5. doi: 10.1016/j.diabres.2014.04.016. [Epub ahead of print]

Brown DW, Croft JB, Greenlund KJ, Giles WH. Trends in hospitalization with chronic obstructive pulmonary disease-United States, 1990-2005.COPD. 2010 Feb;7(1):59-62. doi: $10.3109 / 15412550903499548$.

Carvalho, F., Barros, D., Silva, J., Rezende, E., Soares, M., Fregoneze, J., \& De Castro, e. S. (2004). Hyperglycemia induced by acute central fluoxetine administration: Role of the central CRH system and 5-HT3 receptors. Neuropeptides, 38(2-3), 98-105.

Cazzola, M., Calzetta, L., Bettoncelli, G., Cricelli, C., Romeo, F., Matera, M. G., \& Rogliani, P. (2012). Cardiovascular disease in asthma and COPD: A population-based retrospective crosssectional study. Respiratory Medicine, 106(2), 249-256. doi:10.1016/j.rmed.2011.07.021; 10.1016/j.rmed.2011.07.021

Cazzola, M., Ciaprini, C., Page, C. P., \& Matera, M. G. (2007). Targeting systemic inflammation: Novel therapies for the treatment of chronic obstructive pulmonary disease. Expert Opinion on Therapeutic Targets, 11(10), 1273-1286.

CDC. (2012). Chronic obstructive pulmonary disease among adults - united states, 2011. (Morbidity and Mortality Weekly Report). Atlanta, GA: Centers for Disease Control and Prevention. 
Colbert, J. D., \& Stone, J. A. (2012). Statin use and the risk of incident diabetes mellitus: A review of the literature. The Canadian Journal of Cardiology, 28(5), 581-589.

doi:10.1016/j.cjca.2012.03.021

Department of Health and Human Services. (2014) Federal Register; Vol 79. Proposed Rules. http://www.gpo.gov/fdsys/pkg/FR-2014-07-11/pdf/2014-15948.pdf; Accessed Aug 21, 2014

Fabbri, L. M., \& Rabe, K. F. (2007). From COPD to chronic systemic inflammatory syndrome? Lancet, 370(9589), 797-799.

Fabbri, L. M., Luppi, F., Beghé, B., \& Rabe, K. F. (2008). Complex chronic comorbidities of COPD. The European Respiratory Journal, 31(1), 204-212. doi:10.1183/09031936.00114307

Ford, E. S., Croft, J. B., Mannino, D. M., Wheaton, A. G., Zhang, X., \& Giles, W. H. (2013). Chronic obstructive pulmonary disease surveillance-united states, 1999-2011. Chest,

GOLD (2013). Global strategy for the diagnosis, management, and prevention of chronic obstructive pulmonary disease. Available from: http://www.goldcopd.org/guidelines-global-strategy-fordiagnosis-management.html. Accessed Jul 17, 2013.

Hegewald MJ1, Crapo RO. Socioeconomic status and lung function. Chest. 2007 Nov;132(5):160814.

Lin, P., Shaya, F. T., \& Scharf, S. M. (2010). Economic implications of comorbid conditions among medicaid beneficiaries with COPD. Respiratory Medicine, 104(5), 697-704. doi:10.1016/j.rmed.2009.11.009

Mercer SW, Smith SM, Wyke S, O'Dowd T, Watt GC.Multimorbidity in primary care: developing the research agenda. Fam Pract. 2009 Apr;26(2):79-80. doi: 10.1093/fampra/cmp020.

NHLBI. (2009). Morbidity and mortality: 2009 chartbook on cardiovascular, lung and blood diseases. (). Washington, SC: U.S. Department of Health and Human Services. National Institutes of Health.

O'Byrne, ,P.M., Rennard, S., Gerstein, H., Radner, F., Peterson, S., Lindberg, B., . . . Sin, D. D. (2012). Risk of new onset diabetes mellitus in patients with asthma or COPD taking inhaled corticosteroids. Respiratory Medicine, 106(11), 1487-1493. doi:10.1016/j.rmed.2012.07.011

Rabe, K. F., Hurd, S., Anzueto, A., Barnes, P. J., Buist, S. A., Calverley, P., . . Global Initiative for Chronic Obstructive Lung Disease. (2007). Global strategy for the diagnosis, management, and prevention of chronic obstructive pulmonary disease: GOLD executive summary. American 
Journal of Respiratory and Critical Care Medicine, 176(6), 532-555.

doi:10.1164/rccm.200703-456SO

Sattar, N., Preiss, D., Murray, H. M., Welsh, P., Buckley, B. M., de Craen, A.,J.M., . . Ford, I. (2010). Statins and risk of incident diabetes: A collaborative meta-analysis of randomised statin trials. Lancet, 375(9716), 735-742. doi:10.1016/S0140-6736(09)61965-6

Schnell, K., Weiss, C. O., Lee, T., Krishnan, J. A., Leff, B., Wolff, J. L., \& Boyd, C. (2012). The prevalence of clinically-relevant comorbid conditions in patients with physician-diagnosed COPD: A cross-sectional study using data from NHANES 1999-2008. BMC Pulmonary Medicine, 12, 26-26. doi:10.1186/1471-2466-12-26

Schonbeck, U., \& Libby, P. (2004). Inflammation, immunity, and HMG-CoA reductase inhibitors: Statins as antiinflammatory agents? Circulation, 109(21 Suppl 1), II18-26.

doi:10.1161/01.CIR.0000129505.34151.23

Suissa, S., Kezouh, A., \& Ernst, P. (2010). Inhaled corticosteroids and the risks of diabetes onset and progression. The American Journal of Medicine, 123(11), 1001-1006.

doi:10.1016/j.amjmed.2010.06.019

Takahashi, S., Nakamura, H., Seki, M., Shiraishi, Y., Yamamoto, M., Furuuchi, M., .. . Ishizaka, A. (2008). Reversal of elastase-induced pulmonary emphysema and promotion of alveolar epithelial cell proliferation by simvastatin in mice. American Journal of Physiology.Lung Cellular and Molecular Physiology, 294(5), L882-L890. doi:10.1152/ajplung.00238.2007

Walsh, G. M. (2008). Statins as emerging treatments for asthma and chronic obstructive pulmonary disease. Expert Review of Respiratory Medicine, 2(3), 329-335. doi:10.1586/17476348.2.3.329 
CHAPTER 2 


\section{CHAPTER 2: INFLAMMATION-RELATED MULTIMORBIDITY AND COPD MANAGEMENT AMONG MEDICAID BENEFICIARIES WITH NEWLY-DIAGNOSED COPD}

\section{INTRODUCTION}

Multimorbidity is defined as coexistence of two or more chronic conditions. (C. M. Boyd, Ritchie, Tipton, Studenski, \& Wieland, 2008; M. Fortin, Soubhi, Hudon, Bayliss, \& van den Akker, 2007; Valderas, Starfield, Sibbald, Salisbury, \& Roland, 2009) Agency for Healthcare Research and Quality suggests that multimorbidity affects care of each chronic condition by complications posed by each condition on another such as interaction between medications or disabling impact of one condition on another.(AHRQ, 2007) Multimorbidity is a major challenge among elderly individuals aged 65 or older. (Marengoni et al., 2011) However, in recent years multimorbidity is no longer considered as just an older person's issue due to its high prevalence among younger adults aged less than 65 years. (Naessens et al., 2011; Taylor et al., 2010) The prevalence of multimorbidity in the Australian population is estimated to be $15 \%$ among younger adults in the age group $40-59$ years. (Taylor et al., 2010) In the United States (US) among adults participating in the National Health Interview survey and in the age group 45-64 years, the prevalence of multimorbidity has been increasing and has grown from $24.5 \%$ in 2004 to $28.1 \%$ in 2010. (Ward \& Schiller, 2013)A retrospective cohort study of 33,324 employees and dependents of the Mayo clinic (aged 18-64 years) found that $54.3 \%$ of the total population had two or more chronic conditions and $16.5 \%$ had five or more chronic conditions. (Naessens et al., 2011)

\section{Multimorbidity and Young Adults}

Although multimorbidity is highly prevalent among younger adults, only a handful of studies have examined the impact of multimorbidity on health outcomes such as healthcare expenditures 
(Naessens et al., 2011), and health-related quality of life (HRQol) (M. Fortin, Dubois, Hudon, Soubhi, \& Almirall, 2007) among younger adults. In these few studies it has been suggested that adults with multimorbidity pose significant economic burden that persists over a long-term.

(Anderson, 2010; Coughlin \& Long, 2009; Naessens et al., 2011) A study conducted using data from employees of large self-funded health plan indicated that on a per-capita basis, younger adults with multimorbidity ( $\$ 21,182$ among those with 5 or more chronic conditions) had higher healthcare expenditures compared to those without multimorbidity $(\$ 3,952$ among those with single chronic condition). (Naessens et al., 2011) Such higher healthcare expenditures also persisted over all four years of the study period. (Naessens et al., 2011) Similarly, in a report published by the Robert Wood Johnson Foundation, it has been indicated that more than three-fifths of healthcare spending on the overall US population is for individuals with multimorbidity. (Anderson, 2010) These findings are in conclusion with another study on multimorbidity and healthcare expenditures among high-cost Medicaid beneficiaries. (Coughlin \& Long, 2009) Furthermore, the adverse impact of multimorbidity has also been observed in terms of healthcare utilization. In 2009, of an estimated 326 million physician office visits, $39.4 \%$ visits were made by adults in the age group 45-64 and with multimorbidity, suggesting that young adults had higher healthcare utilization compared to those without multimorbidity. (Ashman \& Beresovsky, 2013) In addition, almost a quarter of individuals in age group 45-64 with multimorbidity delayed needed medical care due to cost considerations (Freid, Bernstein, \& Bush, 2012), highlighting compromised access for individuals with multimorbidity.

In addition to higher rates of healthcare utilization and expenditures, multimorbidity also affects the quality of life among younger adults. It has been reported that among younger adults with multimorbidity (cardiac and respiratory conditions) HRQoL was significantly lower when measured 
in terms of SF-36 physical component summary compared to those without multimorbidity (mild or no problems with cardiac and respiratory disorders). (M. Fortin et al., 2007)

These studies highlight that there exists knowledge gap in terms of the comprehensive understanding of multimorbidity and its impact on management of specific chronic conditions, healthcare utilization and disease-specific outcomes among younger adults.

\section{Multimorbidity among Adults with Chronic Obstructive Pulmonary Disease (COPD)}

In many studies that estimate the prevalence and patterns of multimorbidity among adults aged over 55 years, COPD is often included as one of the chronic conditions (Britt, Harrison, Miller, \& Knox, 2008) suggesting that multimorbidity may be a common problem among individuals with COPD. It is important to analyze the impact of multimorbidity among individuals with COPD for many reasons. First, COPD is highly prevalent in the United States (US). As of 2011, approximately 15 million Americans (6.3\%) have diagnosed COPD; however, it has been reported that equivalent number of Americans may have undiagnosed COPD. (CDC, 2012; Ford et al., 2013) Second, COPD is a highly debilitating condition associated with significantly poor health-related quality of life (Ryynänen, Soini, Lindqvist, Kilpeläinen, \& Laitinen, 2013) and is the third leading cause of mortality in the United States. In addition to the clinical burden of COPD, it is also associated with high economic (NHLBI, 2009) and social burden (Lopez \& Mathers, 2006). Third, the overall management of COPD accounts for $\$ 49.5$ billion. (NHLBI, 2009)Fourth, compared to individuals without COPD, individuals with COPD are at a higher risk of having multimorbidity due to common inflammation-related pathways. (Fabbri, Luppi, Beghé, \& Rabe, 2008)Therefore, an understanding of prevalence of multimorbidity and its impact on health outcomes among individuals with COPD is an important first step towards improving healthcare management of individuals with COPD. 


\section{Prevalence of Inflammation-related multimorbidity among individuals with COPD}

When examining multimorbidity among individuals with COPD, it is important to note that COPD is mainly characterized as an inflammatory disease that is associated with progressive and persistent airflow limitation. (Rabe et al., 2007) Therefore, it has been suggested that there may be high prevalence of inflammation-related chronic conditions among individuals with COPD due to elevated levels of inflammatory mediators. Although, the exact mechanisms have yet not been established, researchers suggest that inflammation in COPD may either have a "spill-over" effect or the inflammation may be a part of "systemic" inflammation. (Fabbri et al., 2008)A review on systemic manifestations of COPD suggested that systemic inflammation in COPD may lead to various inflammation-related chronic diseases such as arthritis, cardiovascular diseases, depression, diabetes and osteoporosis. (Barnes \& Celli, 2009; Nussbaumer-Ochsner \& Rabe, 2011) Indeed, researchers have suggested that COPD should be deemed as part of "chronic systemic inflammatory syndrome”. (Barnes \& Celli, 2009)

Although there are no specific studies of inflammatory-related multimorbidity among individuals with COPD, a study using data from the National Health and Nutrition Examination Survey (1999-2008) reported that among individuals greater than 45 years old, those with COPD had significantly higher rates of coexisting arthritis (54.6\% vs. $36.9 \%)$, diabetes (16.3\% vs. $12.8 \%)$, depression (20.6\% vs. $12.5 \%)$, osteoporosis (16.9\% vs. $8.5 \%)$, coronary heart disease (12.7\% vs. $6.1 \%)$, congestive heart failure (12.1\% vs. $3.9 \%)$, and stroke ( $8.9 \%$ vs. $4.6 \%)$ as compared to those without COPD. (Schnell et al., 2012) These findings suggest that inflammatory conditions (arthritis, cardiovascular diseases, depression, diabetes and osteoporosis) may be highly prevalent among those with COPD. 


\section{Challenges to COPD Management}

The management of individuals with COPD primarily revolves around pharmacologic treatments. The main medications used in the management of COPD include bronchodilators and corticosteroids. Primary goals of the COPD management comprise of reducing the symptoms of COPD and decreasing/minimizing the risk associated with COPD. (Rabe et al., 2007)

Inflammatory-related multimorbidity among adults with COPD may complicate overall COPD management. (Tsiligianni, Kosmas, Van, \& Tzanakis, 2013) One complication may arise because pharmacological therapies for COPD may interact or may have contraindications with therapies for inflammatory-related conditions. For example, beta blockers which are used to treat cardiovascular conditions may interact with beta agonists, main pharmacological therapy for COPD management. (van der Woude et al., 2005) Similarly, systemic corticosteroids to treat individuals with severe COPD have been linked to impairment in glucose metabolism process and thus may affect diabetes outcomes among individuals with COPD and diabetes. (Caughey, Preiss, Vitry, Gilbert, \& Roughead, 2013) Another complication may arise because presence of some conditions such as CVD can affect metabolism of COPD-medications. For example, theophyline clearance is reduced among individuals with COPD and CVD and may lead to poor COPD-specific outcomes. (Ogawa, Stachnik, \& Echizen, 2013)

Although pharmacological therapy is the hallmark of COPD management, there are no clinical practice guidelines on how to manage COPD in the presence of multimorbidity. In fact, even among older adults with very high prevalence of multimorbidity there have been no established clinical practice guidelines. (C. M. Boyd et al., 2005)

In the absence of clinical practice guidelines for individuals with multimorbidity the treatment for chronic conditions are at the discretion of clinical decision making by the healthcare 
providers. Therefore, it is important to understand the current treatment practice among individuals with COPD and multimorbidity in order to improve their healthcare management.

\section{Impact on Overall Health Outcomes}

In addition to challenges in COPD management, inflammatory-related multimorbidity may also affect overall health and COPD-specific health outcomes of individuals with COPD. Although, the impact of inflammation-related multimorbidity has not been specifically studied among younger adults with COPD, some studies have evaluated overall mortality, healthcare utilization, and healthcare expenditures associated with multimorbidity among individuals with COPD of all ages. It has been reported that among individuals with COPD, inflammation- related multimorbidity are associated higher likelihood of all-cause mortality. (Antonelli Incalzi et al., 1997; McGhan et al., 2007)

Higher overall healthcare utilization and expenditures have been observed among individuals with COPD compared to those without COPD. Findings from a recent study suggest that presence of multimorbidity was associated with significantly higher overall medical utilization and costs among Medicaid beneficiaries with COPD (cases) as compared to matched controls. For example, the highest medical utilization as measured by number of service claims was higher among individuals with COPD and diabetes as compared to matched controls with diabetes (148.3 vs. 81.5, p < 0.001). (Lin, Shaya, \& Scharf, 2010) Although, the impact of inflammation-related multimorbidity on general health outcomes has been studied, the relationship between inflammationrelated multimorbidity and COPD-specific outcomes is an understudied area.

\section{Effects on COPD-Specific Outcomes}

Only a few studies have examined the relationship between multimorbidity on COPDspecific outcomes. One study evaluated the risk of COPD-related re-hospitalizations associated with co-morbidities among individuals of all ages with COPD. The results revealed that COPD related re- 
hospitalizations were higher among individuals with pulmonary hypertension (HR: 1.24; 95\% CI: 1.14 - 1.35). However, contrary to our understanding, presence of diabetes (HR: 0.76; 95\% CI: 0.63 - 0.91) or hypertension (HR: 0.77; 95\% CI: $0.66-0.90$ ) was associated with lower likelihood of COPD-related re-hospitalizations. (McGhan et al., 2007) A study using data from 9,716 patients of all ages, admitted with COPD, across National Health Services Trusts in the United Kingdom (UK) evaluated the relationship between multimorbidity and COPD related outcomes. This study reported that other than the lung-related multimorbidity such as lung cancer, cardiovascular diseases and diabetes were significantly association with increased risk of 90-day readmissions. For example, individuals with ischemic heart disease had 20\% increased risk of 90-day COPD readmission (RR: 1.20, 95\% CI: 1.12 - 1.27). (Roberts, Stone, Lowe, Pursey, \& Buckingham, 2011)

\section{Need for the study}

As suggested in the introduction section, studies related to impact of multimorbidity on health outcomes have mostly focused on elderly individuals; specifically, elderly Medicare beneficiaries. Evidence has accumulated that multimorbidity is common among young adults and it is important to understand the relationship between multimorbidity and health outcomes in this population.

Among the younger adults population, enrollees in the Medicaid population due to their low socioeconomic status generally tend to have poor health outcomes. As confirmed by a recently conducted systematic review low socio-economic status is associated with higher rates of COPD prevalence and poor health outcomes such as mortality and higher healthcare utilization. (A. S. Gershon, Dolmage, Stephenson, \& Jackson, 2012) In the United States, Medicaid is an important payer of a major part of young adult population. (KFF, 2011) Furthermore, it also represents individuals in the lower socio-economic strata of the society. (KFF, 2011) Studies have shown that multimorbidity has worse impact among individuals in the low SES category compared to those in 
higher socioeconomic groups. (Sawa, 2011) In terms of Medicaid beneficiaries with COPD, investigators have indicated that individuals with COPD have more rates of multimorbidity compared to matched non-COPD counterparts. Utilizing the Maryland Medicaid database, Lin et al reported that beneficiaries with COPD had higher prevalence of diabetes mellitus (27.7\%), congestive heart failure (17.9\%) and cerebrovascular disease (10.7\%). (Lin et al., 2010) The high prevalence of COPD and multimorbidity in the Medicaid population necessitates the examination of impact of multimorbidity on COPD-specific outcomes among Medicaid beneficiaries with COPD.

When considering individuals with multimorbidity, the Wagner chronic care model of structured care suggests that evidence based support for decision making and the use of clinical guidelines are key elements in providing high quality and effective care. (Wagner et al., 2005) Indeed, an expert review on guiding principles for care of adults with multimorbidity has highlighted the importance of availability of relevant evidence regarding important outcomes. (Guiding principles for the care of older adults with multimorbidity: An approach for clinicians: American geriatrics society expert panel on the care of older adults with multimorbidity.2012) Despite high prevalence of inflammation-related multimorbidity among individuals with COPD, no clear clinical guidelines for COPD have been established. Therefore, a systematic understanding of COPD and the relationship between inflammation-related multimorbidity and COPD-specific outcomes is required in order to provide evidence based care to individuals with COPD. Furthermore, a comprehensive understanding of the relationship will help in developing interventions which could further improve health outcomes among individuals with COPD. In fact, a systematic review of interventions for people with multimorbidity has suggested that interventions targeted at specific combinations of common conditions, or specific problems for patients with multiple conditions, may be more 
effective. (Smith, 2012) Studies are therefore required to understand the impact of multimorbidity on COPD-specific outcomes.

Only a few studies have assessed the impact of multimorbidity on COPD-specific outcomes. Moreover, there are several limitations present in these studies such as discrete approach followed in terms of assessing multimorbidity. (McGhan et al., 2007; Roberts et al., 2011) As COPD is increasingly being identified as a part of "chronic systemic inflammatory syndrome", (Fabbri et al., 2008) a holistic approach is required to understand the relationship between multimorbidity and COPD. In addition, the population included in one study had individuals with asthma which may have led to misdiagnosis of COPD. Some of the other limitations of the studies presented in our introductions section include no adjustment of potential confounders such as co-medications.

The high prevalence of inflammation-related multimorbidity among individuals with COPD and dearth of literature in terms of its impact on COPD-specific outcomes necessitates a comprehensive investigation. In addition, it is important to understand the relationship between inflammation-related multimorbidity and COPD among vulnerable Medicaid beneficiaries after extensively controlling for potential confounders. Therefore, our study aims to fill the critical knowledge gaps in the area of multimorbidity among younger adults with COPD.

A unique contribution of our study is to have a better understanding of a relationship between inflammation-related multimorbidity and COPD-specific outcomes. COPD is often understood a disease with local inflammation. Therefore, most pharmacologic medications used in treating COPD focuses on reducing local inflammation and thereby improving only symptoms and not changing the course of disease progression. Recently, investigators have begun to consider COPD as a disease associated with systemic inflammation. By explaining the link between inflammation-related multimorbidity and COPD-specific outcomes, we strengthen the current understanding about 
inflammation in COPD and provide a novel target area (systemic inflammation) to improve COPDspecific outcomes. 


\section{CONCEPTUAL FRAMEWORK}

For the purposes of this study we adapted the conceptual framework from "Competing demands" model and operationalized the framework based upon our study requirements. The diagram (figure 1) below details the basic version of the "competing demands" model originally used in assessing the impact of multimorbidity on preventive/primary care. (Jaén, Stange, \& Nutting, 1994) It was later adapted by Klinkam et al in understanding the relationship between multimorbidity and care among individuals with depression. (Klinkman, 1997) The model described below contains four specific domains: a) the patient; b) the clinician; c) the practice ecosystem and d) the overall policy environment.

The interplay between these characteristics eventually affects the care being provided to the patient. We were not able to determine any clinician characteristics due to unavailability of data. Competing demands from multimorbidity may lead to poorer COPD-specific outcomes compared to those without multimorbidity.

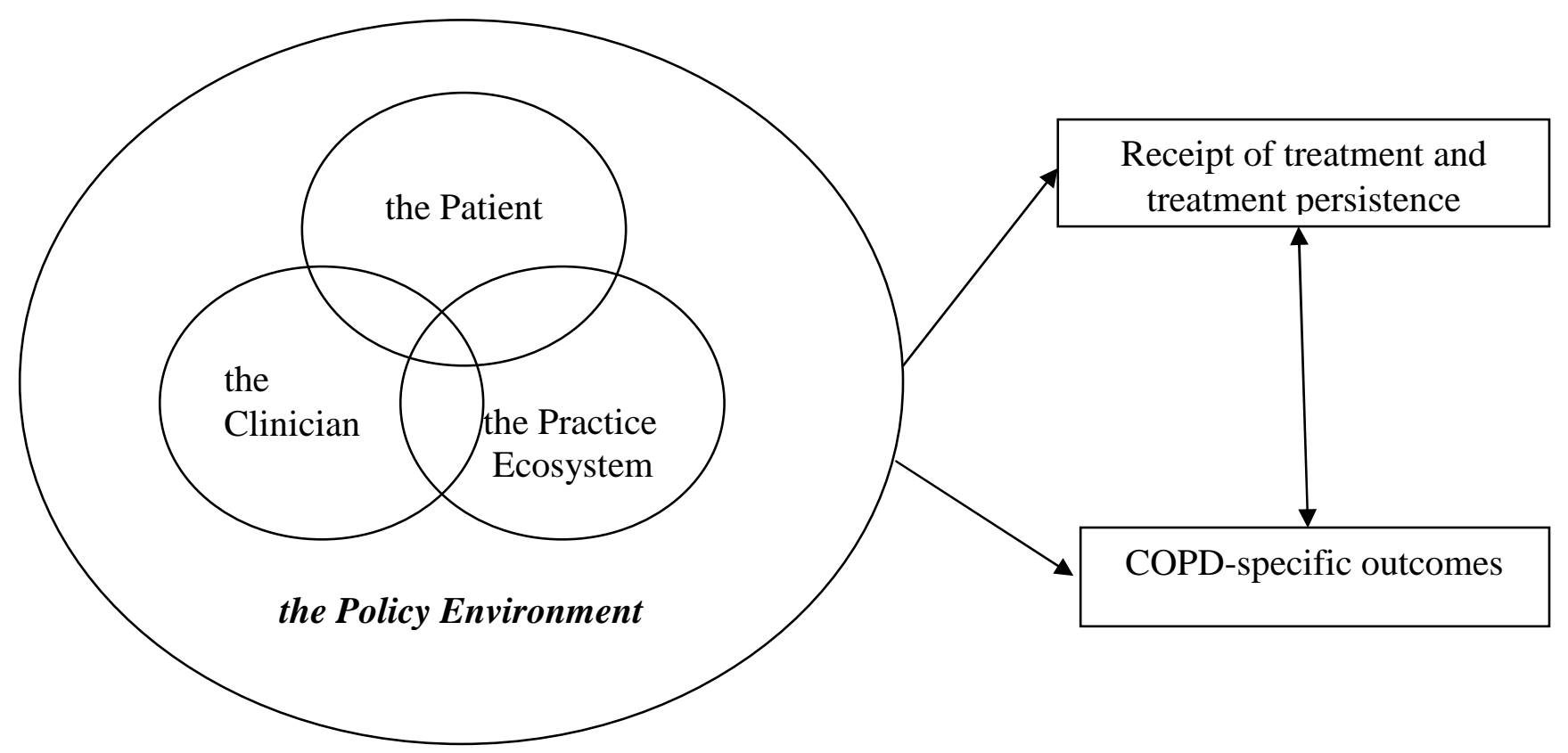

Figure 1: “Competing Demands” Model on Impact of Multimorbidity on Health Outcomes 


\section{METHODS}

\section{Study design}

We utilized a retrospective longitudinal dynamic cohort design using administrative claims data for the years 2005 through 2008. Individuals with COPD were identified between 01/01/2006 and 12/31/2007 (Index date Identification Period). Index date was recorded for each Medicaid beneficiary as the first observed inpatient or outpatient service date with COPD diagnosis. Baseline period was considered as 12 months prior to this index date and follow up period was considered as 12 months after the index date.

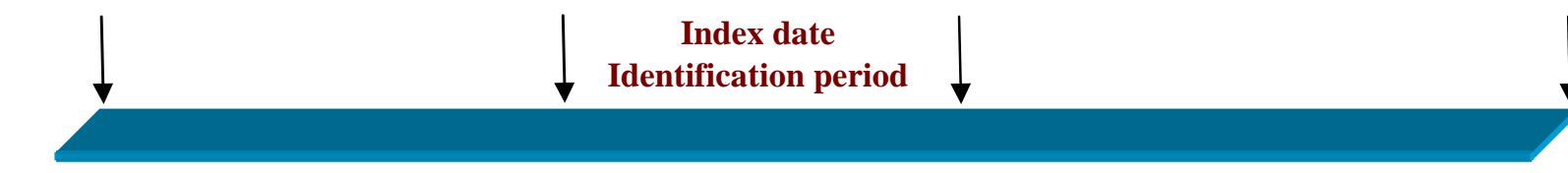

Baseline period (1 year prior to index date)

$01 / 01 / 2005$

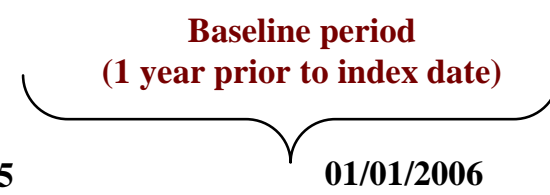

Follow-up period I (1 year from end index date)

12/31/2007

$12 / 31 / 2008$

Figure 2: An example of description of study periods

\section{Data Source}

\section{Medicaid Analytic eXtract (MAX) (2005-2008)}

Medicaid analytic extract (MAX) files are prepared and produced by the Centers for Medicare and Medicaid Services with the help of Research and Data Assistance Center (ResDAC) for performing research on healthcare utilization and outcomes among Medicaid beneficiaries. States administering the Medicaid program are required to report annual, person-level data via Medicaid Statistical Information System (MSIS) for the Medicaid beneficiaries to the CMS. These person level files provide information about beneficiaries' eligibility, their healthcare utilization and payment information. Several initiatives are taken by the agencies to maintain and improve the 
quality of these datasets. (CMS, 2011; Hennessy, Leonard, Palumbo, Newcomb, \& Bilker, 2007)We received separate files from CMS which were linked based on beneficiaries' unique identification numbers. These included: enrollment (“personal summary”), inpatient and outpatient medical claims, and pharmacy claims files for beneficiaries.

The personal summary file provided information on eligibility, demographics (age, gender, and race), managed care enrollment, and utilization summary. The other four files contained information on fee-for-service claims for services used. “Other therapy” included information regarding claims for Medicaid services provided at an outpatient level such as clinic services, physician services, etc. Information regarding services provided during hospitalizations was obtained from the “Inpatient file". These files contain data such as hospitalization/outpatient services diagnoses codes, procedures, length of stay for each hospitalization and expenditures related to hospitalizations or other services. All the information regarding pharmacy or drug services was obtained from the “Prescription Drug” file. This file included information regarding utilization of prescription drugs by Medicaid beneficiaries including prescription fill date, days of supply, national drug code (NDC).

We used data for beneficiaries residing in the following states: California (CA), Illinois (IL), New York (NY) and Texas (TX). The primary reason behind using this data was to obtain programs with lower Medicaid-managed care penetration rates so that more utilization characteristics would be captured. This provided us the ability to obtain maximum number of beneficiaries with fee-forservice enrollments. 


\section{Area Resource File (ARF):}

In addition to all the demographic characteristics and utilization variables obtained from the MAX files, we also determined each Medicaid beneficiary's contextual county level variables using the Area Resource File (ARF). ARF is a comprehensive county-level dataset compiled by the Health Resources Service Administration's Bureau of Health Professions. ARF contains more than 6000 variables providing information about nation's counties. The information contained within ARF includes type of health facilities in the various counties, number and type health professions, resource scarcity measures, health status, economic activity, health training programs, and socioeconomic and environmental characteristics. These files also include county codes and descriptors that allow its linkage with several secondary datasets such as MAX. The type of variables that were obtained from ARF included density of pulmonologist in beneficiary's residing county. We used the county codes and state information to link MAX files with 2008 ARF file. All the ARF variables were obtained from the baseline period.

\section{Study Population}

\section{Medicaid Beneficiaries with newly-diagnosed COPD}

Medicaid beneficiaries with diagnosed COPD were identified using MAX IP and OT files (physician office visits claims only). Baseline period were used to identify Medicaid Beneficiaries with COPD. Individuals with at least one inpatient visit or two 14 days apart outpatient visits (obtained using type of service codes) for COPD based on International Classification of Diseases, Ninth Revision, Clinical Modification (ICD-9-CM) codes for chronic bronchitis (491.xx), emphysema (492.xx), or unspecified chronic airway obstruction (496.xx) were considered to have diagnosed COPD. These diagnosis codes have been utilized in prior published research (codes 491.xx, 492.xx and 496.xx) to identify COPD and evaluate medical treatment and health outcomes among individuals with COPD. (Dalal, Shah, D'Souza, Chaudhari, \& Crater, 2012; Halpern et al., 
2011; Make, Dutro, Paulose-Ram, Marton, \& Mapel, 2012) Indeed, sensitivity and specificity of using ICD-9-CM codes to identify patients with COPD has been established. (Cooke et al., 2011; A. S. Gershon et al., 2009) A study conducted by Gershon et al reported that identifying COPD using 1 or more ambulatory claims and/or 1 or more hospitalizations for COPD resulted in a sensitivity of 85.0\% (95\% CI: 77.0 to 91.0 ) and a specificity of $78.4 \%$ (95\% CI: 73.6 to 82.7 ). However, we will be used 1 inpatient or 2 outpatient claims to identify individuals with COPD in order to increase the specificity of the algorithm.

Other inclusion criteria will included: a) 40-64 years (among young adults this age group is at highest risk of COPD); b) continuous eligibility during the baseline and follow-up period; c) no dual Medicaid/Medicare coverage (dual eligibility will represent high-cost and severe beneficiaries); d) enrolled in fee-for-service plans throughout the study observation period; e) Alive during the study observation period; and use of services (inpatient or outpatient).

\section{Dependent Variables}

\section{Medication Receipt (Objective 1.1)}

According to various clinical practice guidelines pharmacologic treatment for individuals with COPD should include administration of maintenance bronchodilators and inhaled corticosteroids. Medication use among individuals with COPD was identified using the NDCs obtained from variety of sources including Food and Drugs Administration (FDA) register.

Following indicator variables will be created

i) Receipt of short-acting bronchodilators including beta agonists and inhaled anticholinergics (yes/no)

ii) Receipt of long-acting bronchodilators including beta agonists and inhaled anticholinergics (yes/no) 
iii) Receipt of any bronchodilator (short-/long-acting) categorized into four groups: 1)Shortacting bronchodilator only; 2) long-acting bronchodilator only; 3) both; 4) none

iv) Receipt of inhaled corticosteroid (yes/no)

\section{Long-Acting Bronchodilators: Treatment Persistence (Objective 1.1):}

Long-acting bronchodilators are considered as maintenance medications for individuals with COPD. Therefore, in addition to the receipt of medications, it is important to determine the impact of inflammation-related multimorbidity on the persistence with long-acting bronchodilators. We measured 180-day persistence among individuals who initiated with long-acting bronchodilators in the first 6-months of the follow-up period. Medication persistence was defined as continuous use i.e. without a 45-day gap in prescription refills of long-acting bronchodilators. Sensitivity analyses were conducted with varying gap periods i.e. 30-day gap (rigid measure) and 60-day gap (flexible measure). Time to discontinuation was calculated by subtracting the first discontinuation date (first date after initiation of pre-specified gap period) from the index date. For individuals who did not discontinue the therapy during the 180-day observation period, time to discontinuation was recorded as 180 days.

\section{COPD-Specific healthcare utilization: (Objective 1.2)}

Any healthcare claim with primary diagnosis of COPD identified using ICD-9-CM codes were considered as COPD-specific health care utilization. Following variables were identified as COPD-specific health care utilization: a) COPD-specific hospitalizations (yes/no), b) COPD-specific Emergency Room visits (yes/no); obtained from and inpatient and outpatient claims and c) COPDspecific outpatient visits (high density/low density) determined by categorizing the number of COPD-specific outpatient visits above and below the median.

COPD-Related healthcare utilization: (Objective 1.2) 
Healthcare claims with any COPD diagnosis identified using ICD-9-CM codes were considered as COPD-related health care utilization. Following variables were identified as COPDrelated health care utilization: a) COPD-related hospitalizations (yes/no), b) COPD-related Emergency Room visits (yes/no); obtained from and inpatient and outpatient claims

\section{COPD-specific healthcare expenditures (Objective 1.3)}

We also measured COPD- specific and COPD-related total healthcare, inpatient and outpatient expenditures including expenditures for inpatient, outpatient, and emergency room expenditures. COPD-specific expenditures were identified by identifying service claims with the primary diagnosis of COPD (inpatient and outpatient). To account for medical services inflation, expenditures in 2006 and 2007 were converted to 2009 dollars using the annual consumer price index for medical care services available from the Bureau of Labor Statistics. (BLS, 2013)

\section{Key Independent Variable: Inflammation-related Multimorbidity}

Binary indicator variables were created for the presence of arthritis, CVD, depression, diabetes and osteoporosis using the International Classification of Diagnosis $-9^{\text {th }}$ Revision- Clinical Modification (ICD-9-CM) codes obtained from the Agency for Healthcare Research and Quality (AHRQ) - Clinical Classification Software. Any Medicaid beneficiary with COPD having greater than or equal to one inpatient or two outpatient visits for these conditions were considered as having the disease. In addition indicator variables were created for inflammation-related physical conditions and inflammation-related mental condition. Any Medicaid beneficiary having either of the following conditions: arthritis, CVD, diabetes and osteoporosis were considered as having inflammationrelated physical condition. Similarly, any individual with COPD having depression was considered as having inflammation-related mental condition.

\section{Operational definition:}


Inflammation-related multimorbidity was further categorized into:

1) COPD + Inflammation-related Physical Conditions + Inflammation-related Mental Condition (i.e. depression)

2) $C O P D+$ Inflammation-related Physical Conditions

3) $C O P D$ + Inflammation-related Mental Condition (i.e. depression)

4) COPD only (without any chronic conditions).

\section{Other Independent Variables}

Based on the conceptual framework we have grouped the independent variables into the specific domain:

a) Patient Characteristics: These variables included year of diagnosis (2006 vs. 2007), demographic characteristics such as gender (women, men), race (White, African Americans, Others), age in years (40-49, 50-59, 60-64), poverty eligibility (yes, no), medical eligibility (yes, no), number of other clinical conditions (none, 1-3, 4-6, >6), serious mental illness (yes, no), alcohol abuse (yes, no), substance abuse (yes, no), tobacco use (yes, no) and polypharmacy ( $<10$ drug classes, $\geq 10$ drug classes).

b) Eco-system (county-level) Characteristics: County-level variables from ARF files were used to obtain eco-system characteristics. Quartiles for density of above high school education, unemployment, poverty, primary care providers, and specialist care providers were created. Density of these county level characteristics was calculated by dividing total number of each characteristics by the total county population. This density was further converted into per 1000 people by multiplying 1000 with the density. Other ARF characteristics included pulmonologist density (high vs. low), cardiologist density (high vs. low), etc.

d) Policy Environment: Each state administering Medicaid coverage to eligible beneficiaries has different policies; for example, states have restrictions on medications that can be covered. To 
account for policy differences we included a dummy variable for the states (CA, IL, NY, TX) in our analyses.

\section{Statistical Analyses}

\section{Bivariate Analyses}

Chi-square tests of independence were used to determine subgroup differences in categorical variables by inflammation-related multimorbidity among Medicaid beneficiaries with COPD. Bivariate differences in time to discontinuation of long-acting bronchodilators were tested using Kaplan-Meier estimators and log-rank tests were used to determine statistical significance. To test the relationship between continuous variables (example: healthcare expenditures) and inflammationrelated multimorbidity categories we used F-tests.

\section{$\underline{\text { Multivariate Analyses }}$}

Binary and multinomial logistic regressions were used to examine the relationship between inflammation-related multimorbidity and medication receipt, long-acting bronchodilators persistence and COPD-specific healthcare utilization. Parameter estimates are represented as Adjusted Odds Ratio (AOR) with 95\% confidence intervals (CI). The association between inflammation-related multimorbidity and time to discontinuation of long-acting bronchodilators was examined using Coxproportional hazards regression. Parameters estimates from these regressions are expressed as adjusted hazard ratios (aHR) and 95\% confidence intervals (CI). The relationship between inflammation-related multimorbidity and healthcare expenditures among individuals with COPD were analyzed using generalized lineal model (GLM) with log-link function and gamma distribution. As many Medicaid beneficiaries did not have any COPD-specific healthcare expenditures, we used two-part models. The first part of the model predicted the probability of having healthcare expenditures and the second part of the model included GLM with log-link and gamma distribution 
on healthcare expenditures for beneficiaries who had non-zero expenditures. All analyses were conducted using SAS v9.3 (SAS Institute, Cary, NC).

\section{RESULTS}

The final study population (after applying all exclusion criteria) consisted of 19,060 Medicaid beneficiaries aged between 40-64 years with newly diagnosed COPD. The algorithm used to define the study cohort is shown in Figure 1. Majority of the Medicaid beneficiaries with newly diagnosed COPD were women (59.5\%), Whites (48.6\%), in the age group 50-59 years (48.4\%) and residents of CA $(54.5 \%)$.

\section{I) Prevalence of Inflammation-related Multimorbidity}

Overall, among Medicaid beneficiaries with newly-diagnosed COPD ( $\mathrm{n}=19,060), 48.8 \%$ had inflammation-related physical multimorbidity, 6.7\% had inflammation-related mental multimorbidity, $19.6 \%$ had both and $24.9 \%$ did not have any inflammation-related multimorbidity. With regards to individual inflammation-related chronic conditions among Medicaid beneficiaries with newly-diagnosed COPD, $17.9 \%$ had cardiovascular disease, $19.8 \%$ had diabetes, $12.3 \%$ had hyperlipidemia, $37.8 \%$ had hypertension, $35.8 \%$ had musculoskeletal disorders and $26.3 \%$ had depression.

\section{II) COPD Medication receipt}

Findings from the bivariate and multivariate analyses from analyses on COPD medication receipt with inflammation-related multimorbidity as the key independent variable are depicted in the Table 1 (main manuscript section). Approximately 64\% Medicaid beneficiaries with newlydiagnosed COPD used at least short-acting bronchodilator during the follow-up period. The rates of long-acting bronchodilators and inhaled corticosteroids were $39.2 \%$ and $43.3 \%$ respectively. Medicaid beneficiaries with inflammation-related mental multimorbidity were more likely to receive 
short-acting bronchodilators (OR: 1.16 95\%; CI: 1.02, 1.33), those with both physical and mental condition were less likely to receive long-acting bronchodilators (OR: $0.91 ; 95 \%$ CI: $0.84,1.00$ ) but were more likely to receive inhaled corticosteroids (OR: 1.12 ; $95 \%$ CI: $1.03,1.22)$ compared to those with no multimorbidity.

Findings from multivariable logistic regression on COPD medication receipt among Medicaid beneficiaries with newly diagnosed COPD revealed that those with inflammation-related physical conditions were significantly less likely to receive short-acting bronchodilators (AOR: 0.82; 95\% CI: 0.75, 0.89), long-acting bronchodilators (AOR: 0.86; 95\% CI: 0.79, 0.93) and inhaled corticosteroids (AOR: $0.81 ; 95 \%$ CI: $0.75,0.88$ ) compared to adults with no multimorbidity. Similarly, those with both physical and mental multimorbidity were $26 \%$ less likely to receive shortacting bronchodilators (AOR: $0.74 ; 95 \%$ CI: 0.66, 0.81), $26 \%$ less likely to use to long-acting bronchodilator (AOR: $0.74 ; 95 \%$ CI: $0.67,0.82$ ) and $22 \%$ less likely to use inhaled corticosteroids (AOR: 0.78; 95\% CI: 0.71, 0.86) compared to those with no multimorbidity. In addition, certain patient characteristics, eco-system level variables and policy environment variables were significantly associated with COPD medication receipt.

To understand the overall bronchodilator use among Medicaid beneficiaries with newlydiagnosed COPD, we created an aggregate bronchodilator use variable with four categories (short acting only, long-acting only, both and none). Majority (47.2\%) of beneficiaries with at least one bronchodilator use $(\mathrm{n}=13,310)$ in the follow-up period were in the both bronchodilator use group. Findings from multinomial logistic regressions (Table 2, main manuscript section) revealed that adults with physical and both physical and mental multimorbidity were less likely to receive shortacting bronchodilators only (AOR: 0.88; 95\% CI: 0.79, 0.97; AOR: 0.80; 95\% CI: 0.71, 0.91 respectively) as compared to those with no multimorbidity. Similarly findings were observed for 
receipt of short-acting and long-acting bronchodilators. However, inflammation-related

multimorbidity did not have any statistically significant association with receipt of long-acting bronchodilators only.

\section{III) Long-acting bronchodilator persistence (6 months)}

Out of 6,119 Medicaid beneficiaries who initiated on long-acting bronchodilator therapy during the follow-up period, $30.7 \%(\mathrm{n}=1,880)$ remained persistent (i.e. without any 45-day gap period) through 6 month follow-up after the index date. Subgroup differences in persistence rates by inflammation-related multimorbidity were examined using chi-square tests of independence. These results suggested that there was no difference in terms of overall persistence by inflammation-related multimorbidity. (Table 3, main manuscript section) Sensitivity analyses were performed by changing the allowable gap period to 30 days (strict persistence measure) and 60 days (flexible persistence measure). Similar bivariate findings as those obtained using 45-day gap allowance, were observed in the sensitivity analyses as well.

Interestingly, the findings from the logistic regressions on flexible persistence measure revealed that Medicaid beneficiaries with inflammation-related mental condition were $25 \%$ less likely to be persistent (AOR: 0.75; 95\% CI: 0.60-0.96), whereas those with both physical and mental condition were $19 \%$ less likely to remain persistent (AOR: 0.81 ; 95\%CI: $0.68,0.97$ ) in the 6-month follow-up period. No statistically significant relationships were found between inflammation-related multimorbidity and persistence using 30-day and 45-day gap period.

Kaplan-Meier estimates with log-rank tests and cox-proportional hazards model were conducted to understand the unadjusted and adjusted relationship between inflammation-related multimorbidity and time-to-discontinuation (in days) respectively. Specific findings from these analyses with regards to inflammation-related multimorbidity are presented in Table 3, main manuscript section. Median time-to-discontinuation of long-acting bronchodilators was lower for 
Medicaid beneficiaries with mental condition (96.0 days, 95\%CI: 87.9, 110.1), with physical condition (102.0 days, 95\%CI: 93.9, 107.1) and those with both conditions (107.0 days, 95\%CI: $99.9,114.0)$ compared to those with no multimorbidity (113.0 days, $95 \% \mathrm{CI}: 105.0,117.9)$. However, this difference was not statistically significant. Sensitivity analyses with different allowable gap period revealed similar results.

Cox proportional hazards regression on time-to-discontinuation (in days) indicated that after adjusting for baseline characteristics and using 60-day allowable gap period to measure time-todiscontinuation, individuals with inflammation-related physical condition only (aHR: 1.10; 95\% CI: $1.01,1.20$ ), inflammation-related mental condition only (aHR: 1.18; 95\% CI: 1.03, 1.36) and both conditions (aHR: 1.14; 95\% CI: 1.02, 1.36) had higher hazards of discontinuation as compared to those without any inflammation-related multimorbidity.

IV)COPD-specific and COPD-related healthcare utilization

Table 4 (main manuscript section) depicts the findings from bivariate and multivariate analyses on COPD-specific and COPD-related healthcare utilization. About 5.0\% $(\mathrm{n}=998)$ Medicaid beneficiaries with newly diagnosed COPD had at least 1 COPD-specific hospitalization, $14.8 \%(\mathrm{n}=2,824)$ had COPD-specific emergency room visits and 43.7\% $(\mathrm{n}=7,200)$ had $\geq 1$ COPDspecific outpatient visits. Bivariate analyses did not reveal any statistically significant associations between inflammation-related multimorbidity and COPD-specific healthcare utilization.

However, findings from the logistic regressions that adjusted for baseline characteristics suggested that Medicaid beneficiaries with inflammation-related physical condition only (AOR: 0.81; $95 \%$ CI: $0.68,0.96$ ) and those with both physical and mental condition (AOR: 0.80; 95\% CI: $0.65,0.99)$ were less likely to have COPD-specific hospitalization compared to adults with no multimorbidity. Similar findings were obtained for the relationship between inflammation-related multimorbidity and COPD-specific emergency room visits. 
Overall, $16.0 \%(\mathrm{n}=3,052)$ of beneficiaries had any COPD-related hospitalizations and 21.4\% ( $\mathrm{n}=4,092)$ had COPD-related Emergency Room visits. Statistically significant subgroup differences in rates of COPD-related healthcare utilization by multimorbidity categories were observed. Those with physical condition only (16.0\%), mental condition only (15.4\%) and both $(20.9 \%)$ had significantly $(\mathrm{p}<0.001)$ higher rates of COPD-related hospitalization as compared to those without any inflammation-related multimorbidity (12.4\%). However, adjusting for baseline characteristics showed that only those with both physical and mental conditions had significantly greater likelihood of COPD-related hospitalization (AOR: 1.26; 95\% CI: 1.10, 1.44). Adjusted analyses also indicated that beneficiaries with physical conditions had lower likelihood of COPDrelated Emergency Room visits (AOR: 0.87, 95\% CI: 0.79, 0.95).

\section{V) COPD-specific healthcare expenditures}

Average total of COPD-specific and COPD-related expenditures (referred to as COPD expenditures) in the 12-month follow up period among Medicaid beneficiaries with newly-diagnosed COPD was estimated to be $\$ 3,238$ (SD: $\$ 12,396$ ). Among users, average COPD-specific inpatient expenditures were estimated to be $\$ 7,223$ (SD: $\$ 4,413$ ) and COPD-specific outpatient expenditures were determined to be $\$ 425$ (SD: \$138). Table 5 (main manuscript section) contains mean (SD), median, parameter estimates, standard errors, and statistical significance represented as asterisks for total and types of COPD-specific expenditures by inflammation-related multimorbidity groups. As shown in the table average total COPD-specific expenditures were significantly higher among individuals with inflammation-related physical multimorbidity ( $\$ 3,481$ vs. $\$ 2,872 ; \mathrm{p}<0.01)$. However, COPD-specific inpatient expenditures and COPD-specific outpatient expenditures were not different among different inflammation-related multimorbidity groups.

Findings from GLM with log-link and gamma distribution (Table 5) among those with positive expenditures revealed that total COPD expenditures, COPD-specific inpatient expenditures and 
COPD-specific outpatient expenditures were lower for adults with inflammation-related multimorbidity compared to those with no multimorbidity.

\section{DISCUSSION}

The current study examined the impact of inflammation-related multimorbidity of COPD medication use, long-acting bronchodilator persistence, COPD-specific healthcare utilization and expenditures among Medicaid beneficiaries with newly-diagnosed COPD. In this study cohort approximately three-forth individuals had inflammation-related multimorbidity. Although no study has comprehensively examined the prevalence of inflammation-related comorbidities among individuals with COPD, the rates for some of the individual conditions obtained from our analyses are somewhat consistent with those found in other studies that have evaluated comorbidity burden among individuals with COPD. (Schnell, 2012; Lin, Shaya, \& Scharf, 2010)

In this study, we found that after controlling for patient characteristics, Medicaid beneficiaries with newly-diagnosed COPD were less likely to receive COPD medications including bronchodilators and inhaled corticosteroids. Furthermore, findings from long-acting bronchodilator persistence analyses indicated that individuals with COPD and inflammation-related multimorbidity had increased hazards of discontinuation. It is plausible that due to competing demands from healthcare management of multimorbidity, COPD-medication utilization and treatment persistence may be reduced. Studies have suggested that certain COPD medications are associated with sideeffects that could complicate management of allied co-occurring conditions. For example bronchodilators such as long-acting beta agonists or inhaled anticholinergics are associated with cardiovascular complications such as unstable angina and myocardial infarction. (Au DH, 2004; Lemaitre, 2002; Macie C, 2008; Martin RM, 1998) Similarly, inhaled corticosteroids may increase blood glucose levels among those with pre-diabetes or diabetes. (Suissa S, 2010; Slatore CG, 2009) 
Therefore, it is important to weigh the benefits and safety issues in the healthcare management of patients with COPD and inflammation-related multimorbidity. Clinical practice guidelines need to be developed for individuals with COPD and multimorbidity.

Contrary to our hypotheses, the presence of inflammation-related multimorbidity was associated with lower COPD-specific healthcare utilization. Medicaid beneficiaries with newly-diagnosed COPD and inflammation-related physical conditions or both physical/mental conditions were less likely to have COPD-specific hospitalization and emergency room visits. These findings are consistent with a study that indicated that presence of diabetes and hypertension were associated with lower likelihood of COPD re-hospitalizations (McGhan et al 2007). We can only speculate on the possible reasons behind our study findings. It is possible that those with multimorbidity have greater hospitalizations for other conditions in which COPD may be diagnosed as comorbidity and thus COPD management may take place while being hospitalized for other chronic conditions. This was also reflected in our analyses of COPD-related hospitalizations which were measured as hospitalization with COPD as any of the nine diagnoses codes.

Although inflammation-related physical multimorbidity was associated with higher average total COPD-specific expenditures as compared to those with no multimorbidity ( $\$ 3,482$ vs. $\$ 2,872$; $\mathrm{p}<0.01$ ), this relationship was reversed in multivariable analyses. After controlling for baseline characteristics it was found that inflammation-related multimorbidity was associated with statistically significant lower COPD-specific total, inpatient and outpatient expenditures. Again, these findings highlight the burden of co-occurring conditions in patients with newly diagnosed COPD and inflammation-related multimorbidity. Management of inflammation-related multimorbidity may require resources and these patients may have expenditures that may not be specific to COPD. Indeed, similar evidence has been documented among individuals with mental 
illness among whom multimorbidity is highly prevalent. Among individuals with mental illness a greater proportion of expenditures often relate to conditions not related to mental health. (Banerjea $\mathrm{R}$ et al, 2008)

\section{STRENGTHS AND LIMITATIONS}

The advantages of our study are: the use of administrative claims data which provided us the ability to identify all the medical conditions using diagnosis codes, availability of prescription medication claims, analysis of a comprehensive set of COPD-specific outcomes, examination of a high-risk and low-income population and ability to track capture utilization over time. Our study was strengthened by linking Medicaid files with ARF to comprehensively adjust for healthcare environment that could have affected the relationship between inflammation-related multimorbidity and COPD-specific outcomes. Our study had some limitations such as limited generalizability due to inclusion of beneficiaries with fee-for-service claims only. The study also used data from only four states which may not provide a full understating of overall Medicaid population. We also excluded dual Medicare-Medicaid enrollees from our population which limits the overall generalizability. Due to unavailability of laboratory data we were not able to control for stage of COPD at diagnosis or confirm the diagnosis of COPD.

Despite these limitations, to the best of our knowledge this the first study to extensively examine the relationship between inflammation-related multimorbidity and comprehensive set of COPDspecific outcomes. Therefore, the findings from this study may guide clinical and policy decision making for management of COPD patients with inflammation-related multimorbidity. 


\section{REFERENCES}

AHRQ. (2007). Optimizing prevention and healthcare management for the complex patient.

Retrieved June 10, 2013, from http://archive.ahrq.gov/fund/trans101507.htm

Anderson, G. (2010). Chronic care: Making the case for ongoing care. (). Princeton, NJ: Robert Wood Johnson Foundation.

Antonelli Incalzi, R., Fuso, L., De Rosa, M., Forastiere, F., Rapiti, E., Nardecchia, B., \& Pistelli, R. (1997). Co-morbidity contributes to predict mortality of patients with chronic obstructive pulmonary disease. The European Respiratory Journal, 10(12), 2794-2800.

Ashman, J. J., \& Beresovsky, V. (2013). Multiple chronic conditions among US adults who visited physician offices: Data from the national ambulatory medical care survey, 2009. Preventing Chronic Disease, 10, E64-E64. doi:10.5888/pcd10.120308

Au DH, Udris EM, Curtis JR, et al. Association between chronic heart failure and inhaled beta-2adrenoceptor agonists. Am Heart J 2004; 148: 915-20.

Banerjea R1, Sambamoorthi U, Smelson D, Pogach LM. Expenditures in mental illness and substance use disorders among veteran clinic users with diabetes. J Behav Health Serv Res. 2008 Jul;35(3):290-303. doi: 10.1007/s11414-008-9120-8. Epub 2008 May 30.

Barnes, P. J., \& Celli, B. R. (2009). Systemic manifestations and comorbidities of COPD. The European Respiratory Journal, 33(5), 1165-1185. doi:10.1183/09031936.00128008

Bureau of Labor Statistics. U.S. department of labor, Consumer price index. http://www.bls.gov/cpi/cpiovrvw.htm. Updated 2013. Accessed January, 10, 2014.

Boyd, C. M., Ritchie, C. S., Tipton, E. F., Studenski, S. A., \& Wieland, D. (2008). From bedside to bench: Summary from the american geriatrics Society/National institute on aging research conference on comorbidity and multiple morbidity in older adults. Aging Clinical and Experimental Research, 20(3), 181-188.

Boyd, C. M., Darer, J., Boult, C., Fried, L. P., Boult, L., \& Wu, A. W. (2005). Clinical practice guidelines and quality of care for older patients with multiple comorbid diseases: Implications for pay for performance. JAMA: The Journal of the American Medical Association, 294(6), 716-724.

Britt, H. C., Harrison, C. M., Miller, G. C., \& Knox, S. A. (2008). Prevalence and patterns of multimorbidity in australia. The Medical Journal of Australia, 189(2), 72-77. 
Caughey, G. E., Preiss, A. K., Vitry, A. I., Gilbert, A. L., \& Roughead, E. E. (2013). Comorbid diabetes and COPD: Impact of corticosteroid use on diabetes complications. Diabetes Care,

CDC. (2012). Chronic obstructive pulmonary disease among adults — united states, 2011 . (Morbidity and Mortality Weekly Report). Atlanta, GA: Centers for Disease Control and Prevention.

CMS. (2011). Medicaid analytic eXtract (MAX) general information. (https://www.cms.gov/MedicaidDataSourcesGenInfo/07_MAXGeneralInformation.asp). Baltimore, MD: 50. Centers for Medicare and Medicaid Services, US Department of Health and Human Services.

Cooke, C. R., Joo, M. J., Anderson, S. M., Lee, T. A., Udris, E. M., Johnson, E., \& Au, D. H. (2011). The validity of using ICD-9 codes and pharmacy records to identify patients with chronic obstructive pulmonary disease. BMC Health Services Research, 11, 37-37. doi:10.1186/14726963-11-37

Coughlin, T. A., \& Long, S. K. (2009). Health care spending and service use among high-cost medicaid beneficiaries, 2002-2004. Inquiry: A Journal of Medical Care Organization, Provision and Financing, 46(4), 405-417.

Dalal, A. A., Shah, M., D'Souza, A.,O., Chaudhari, S., \& Crater, G. (2012). Clinical and economic outcomes for patients initiating fluticasone propionate/salmeterol combination therapy (250/50 $\mathrm{mcg}$ ) versus anticholinergics in a comorbid COPD/depression population. International Journal of Chronic Obstructive Pulmonary Disease, 7, 11-19. doi:10.2147/COPD.S27846

Davis, S. N., Wei, W., \& Garg, S. (2011). Clinical impact of initiating insulin glargine therapy with disposable pen versus vial in patients with type 2 diabetes mellitus in a managed care setting. Endocrine Practice : Official Journal of the American College of Endocrinology and the American Association of Clinical Endocrinologists, 17(6), 845-852. doi:10.4158/EP10401.OR; 10.4158/EP10401.OR

Fabbri, L. M., Luppi, F., Beghé, B., \& Rabe, K. F. (2008). Complex chronic comorbidities of COPD. The European Respiratory Journal, 31(1), 204-212. doi:10.1183/09031936.00114307

Ford, E. S., Croft, J. B., Mannino, D. M., Wheaton, A. G., Zhang, X., \& Giles, W. H. (2013). Chronic obstructive pulmonary disease surveillance-united states, 1999-2011. Chest,

Fortin, M., Soubhi, H., Hudon, C., Bayliss, E. A., \& van den Akker, M. (2007). Multimorbidity's many challenges. BMJ (Clinical Research Ed.), 334(7602), 1016-1017. doi:10.1136/bmj.39201.463819.2C 
Fortin, M., Dubois, M., Hudon, C., Soubhi, H., \& Almirall, J. (2007). Multimorbidity and quality of life: A closer look. Health and Quality of Life Outcomes, 5, 52-52.

Freid, V. M., Bernstein, A. B., \& Bush, M. A. (2012). Multiple chronic conditions among adults aged 45 and over: Trends over the past 10 years. NCHS Data Brief, (100), 1-8.

Gershon, A. S., Wang, C., Guan, J., Vasilevska-Ristovska, J., Cicutto, L., \& To, T. (2009). Identifying individuals with physician diagnosed COPD in health administrative databases. COPD, 6(5), 388-394.

Gershon, A. S., Dolmage, T. E., Stephenson, A., \& Jackson, B. (2012). Chronic obstructive pulmonary disease and socioeconomic status: A systematic review. COPD, 9(3), 216-226. doi: $10.3109 / 15412555.2011 .648030$

Guiding principles for the care of older adults with multimorbidity: An approach for clinicians: American geriatrics society expert panel on the care of older adults with multimorbidity. (2012). Journal of the American Geriatrics Society, 60(10), E1-E25. doi:10.1111/j.15325415.2012.04188.x

Halpern, R., Baker, C. L., Su, J., Woodruff, K. B., Paulose-Ram, R., Porter, V., \& Shah, H. (2011). Outcomes associated with initiation of tiotropium or fluticasone/salmeterol in patients with chronic obstructive pulmonary disease. Patient Preference and Adherence, 5, 375-388. doi:10.2147/PPA.S19991

Hennessy, S., Leonard, C. E., Palumbo, C. M., Newcomb, C., \& Bilker, W. B. (2007). Quality of medicaid and medicare data obtained through centers for medicare and medicaid services (CMS). Medical Care, 45(12), 1216-1220. doi:10.1097/MLR.0b013e318148435a

Jaén, ,C.R., Stange, K. C., \& Nutting, P. A. (1994). Competing demands of primary care: A model for the delivery of clinical preventive services. The Journal of Family Practice, 38(2), 166-171.

Kadam, U. (2012). Redesigning the general practice consultation to improve care for patients with multimorbidity. BMJ (Clinical Research Ed.), 345, e6202-e6202. doi:10.1136/bmj.e6202

KFF. (2011). Medicaid matters: Understanding medicaid's role in our health care system. ( No. 8165). Washington, SC: The Henry J Kaiser Family Foundation.

Klinkman, M. S. (1997). Competing demands in psychosocial care. A model for the identification and treatment of depressive disorders in primary care. General Hospital Psychiatry, 19(2), 98111. 
Lemaitre RN, Siscovick DS, Psaty BM, et al. Inhaled beta-2 adrenergic receptor agonists and primary cardiac arrest. Am J Med 2002; 113: 711-6.

Lin, P., Shaya, F. T., \& Scharf, S. M. (2010). Economic implications of comorbid conditions among medicaid beneficiaries with COPD. Respiratory Medicine, 104(5), 697-704. doi:10.1016/j.rmed.2009.11.009

Lopez, A. D., \& Mathers, C. D. (2006). Measuring the global burden of disease and epidemiological transitions: 2002-2030. Annals of Tropical Medicine and Parasitology, 100(5-6), 481-499.

Macie C, Wooldrage K, Manfreda J, et al. Cardiovascular morbidity and the use of inhaled bronchodilators. Int J Chron Obstruct Pulmon Dis 2008; 3: 163-9.

Make, B., Dutro, M. P., Paulose-Ram, R., Marton, J. P., \& Mapel, D. W. (2012). Undertreatment of COPD: A retrospective analysis of US managed care and medicare patients. International Journal of Chronic Obstructive Pulmonary Disease, 7, 1-9. doi:10.2147/COPD.S27032

Marengoni, A., Angleman, S., Melis, R., Mangialasche, F., Karp, A., Garmen, A., . . Fratiglioni, L. (2011). Aging with multimorbidity: A systematic review of the literature. Ageing Research Reviews, 10(4), 430-439. doi:10.1016/j.arr.2011.03.003

Martin RM, Dunn NR, Freemantle SN, et al. Risk of non-fatal cardiac failure and ischaemic heart disease with long acting beta 2 agonists. Thorax 1998; 53: 558-62.

McGhan, R., Radcliff, T., Fish, R., Sutherland, E. R., Welsh, C., \& Make, B. (2007). Predictors of rehospitalization and death after a severe exacerbation of COPD. Chest, 132(6), 1748-1755.

Naessens, J. M., Stroebel, R. J., Finnie, D. M., Shah, N. D., Wagie, A. E., Litchy, W. J., . . Nesse, R. E. (2011). Effect of multiple chronic conditions among working-age adults. The American Journal of Managed Care, 17(2), 118-122.

NHLBI. (2009). Morbidity and mortality: 2009 chartbook on cardiovascular, lung and blood diseases. (). Washington, SC: U.S. Department of Health and Human Services. National Institutes of Health.

Nussbaumer-Ochsner, Y., \& Rabe, K. F. (2011). Systemic manifestations of COPD. Chest, 139(1), 165-173. doi:10.1378/chest.10-1252

Ogawa, R., Stachnik, J. M., \& Echizen, H. (2013). Clinical pharmacokinetics of drugs in patients with heart failure: An update (part 1, drugs administered intravenously). Clinical Pharmacokinetics, 52(3), 169-185. doi:10.1007/s40262-012-0029-2 
Rabe, K. F., Hurd, S., Anzueto, A., Barnes, P. J., Buist, S. A., Calverley, P., . . Global Initiative for Chronic Obstructive Lung Disease. (2007). Global strategy for the diagnosis, management, and prevention of chronic obstructive pulmonary disease: GOLD executive summary. American Journal of Respiratory and Critical Care Medicine, 176(6), 532-555. doi:10.1164/rcem.200703-456SO

Roberts, C. M., Stone, R. A., Lowe, D., Pursey, N. A., \& Buckingham, R. J. (2011). Co-morbidities and 90-day outcomes in hospitalized COPD exacerbations. COPD, 8(5), 354-361. doi:10.3109/15412555.2011.600362

Ryynänen, O., Soini, E. J., Lindqvist, A., Kilpeläinen, M., \& Laitinen, T. (2013). Bayesian predictors of very poor health related quality of life and mortality in patients with COPD. BMC Medical Informatics and Decision Making, 13, 34-34. doi:10.1186/1472-6947-13-34

Schnell, K., Weiss, C. O., Lee, T., Krishnan, J. A., Leff, B., Wolff, J. L., \& Boyd, C. (2012). The prevalence of clinically-relevant comorbid conditions in patients with physician-diagnosed COPD: A cross-sectional study using data from NHANES 1999-2008. BMC Pulmonary Medicine, 12, 26-26. doi:10.1186/1471-2466-12-26

Slatore CG, Bryson CL, Au DH. The association of inhaled corticosteroid use with serum glucose concentration in a large cohort. Am J Med 2009; 122: 472-8.

Suissa S, Kezouh A, Ernst P. Inhaled corticosteroids and the risks of diabetes onset and progression. Am J Med 2010; 123: 1001-6.

Taylor, A. W., Price, K., Gill, T. K., Adams, R., Pilkington, R., Carrangis, N., . . Wilson, D. (2010). Multimorbidity - not just an older person's issue. results from an australian biomedical study. BMC Public Health, 10, 718-718. doi:10.1186/1471-2458-10-718

Tsiligianni, I. G., Kosmas, E., Van, d. M., \& Tzanakis, N. (2013). Managing comorbidity in COPD: A difficult task. Current Drug Targets, 14(2), 158-176.

Valderas, J. M., Starfield, B., Sibbald, B., Salisbury, C., \& Roland, M. (2009). Defining comorbidity: Implications for understanding health and health services. Annals of Family Medicine, 7(4), 357-363. doi:10.1370/afm.983; 10.1370/afm.983

van der Woude, H.,J., Zaagsma, J., Postma, D. S., Winter, T. H., van Hulst, M., \& Aalbers, R. (2005). Detrimental effects of beta-blockers in COPD: A concern for nonselective betablockers. Chest, 127(3), 818-824. 
Wagner, E. H., Bennett, S. M., Austin, B. T., Greene, S. M., Schaefer, J. K., \& Vonkorff, M. (2005). Finding common ground: Patient-centeredness and evidence-based chronic illness care. Journal of Alternative and Complementary Medicine (New York, N.Y.), 11 Suppl 1, S7-S15.

Ward, B. W., \& Schiller, J. S. (2013). Prevalence of multiple chronic conditions among US adults: Estimates from the national health interview survey, 2010. Preventing Chronic Disease, 10, E65-E65. doi:10.5888/pcd10.120203

Wu, E. Q., Birnbaum, H. G., Cifaldi, M., Kang, Y., Mallet, D., \& Colice, G. (2006). Development of a COPD severity score. Current Medical Research and Opinion, 22(9), 1679-1687. doi:10.1185/030079906X115621 


\section{TABLES AND FIGURES}

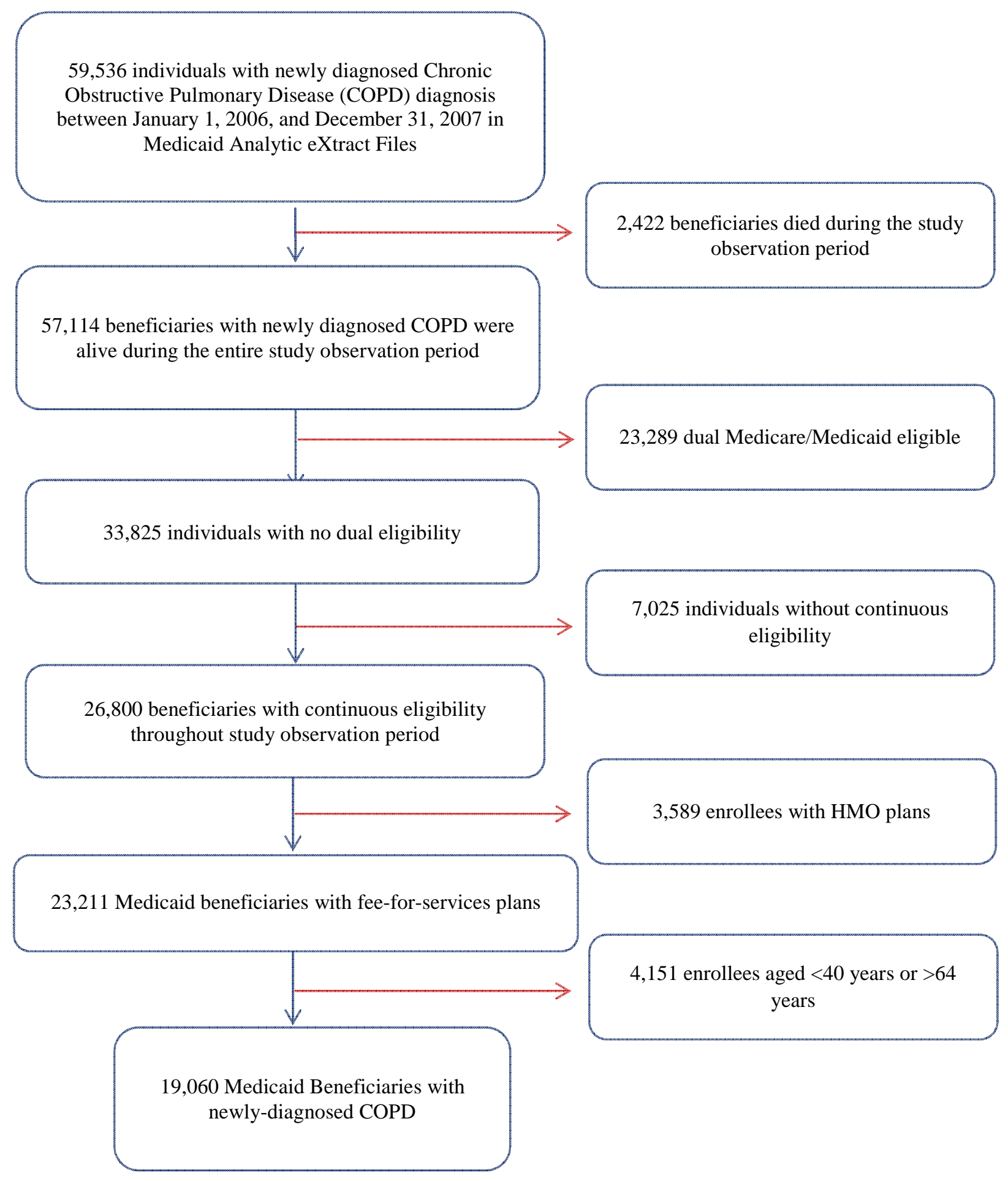

Figure: Algorithm Describing Selection of Study Population 
Table 1

Numbers, Percentages, Odds Ratios, Adjusted Odds Ratios and 95\% Confidence Intervals Receipt of COPD Medication Use among Medicaid Beneficiaries with Newly-Diagnosed COPD

Medicaid Analytic Extract, 2005-2008

\begin{tabular}{|c|c|c|c|c|c|c|c|c|}
\hline \multicolumn{9}{|c|}{ Short Acting Bronchodilator Use } \\
\hline & \multirow[b]{2}{*}{$\mathbf{N}$} & \multirow[b]{2}{*}{ Row \% } & \multicolumn{3}{|c|}{$\begin{array}{l}\text { Unadjusted Logistic } \\
\text { Regression on Receipt of } \\
\text { COPD Medication } \\
\end{array}$} & \multicolumn{3}{|c|}{$\begin{array}{c}\text { Adjusted Logistic Regression } \\
\text { on Receipt of COPD } \\
\text { Medication } \\
\end{array}$} \\
\hline & & & OR & $95 \% \mathrm{CI}$ & Sig & AOR & $95 \% \mathrm{CI}$ & Sig \\
\hline \multicolumn{9}{|c|}{ Short Acting Bronchodilators } \\
\hline TOTAL & 12,177 & 63.9 & & & & & & \\
\hline \multicolumn{9}{|c|}{ Inflammation-related Multimorbidity } \\
\hline Physical Only & 5,947 & 63.9 & 1.05 & {$[0.98,1.13]$} & & 0.82 & {$[0.75,0.89]$} & $* * *$ \\
\hline Mental Only & 850 & 66.3 & 1.16 & {$[1.02,1.33]$} & $*$ & 1.01 & {$[0.88,1.16]$} & \\
\hline Both & 2,407 & 64.5 & 1.08 & {$[0.99,1.18]$} & & 0.74 & {$[0.66,0.81]$} & $* * *$ \\
\hline None & 2,973 & 62.8 & & & & & & \\
\hline \multicolumn{9}{|c|}{ Long Acting Bronchodilators } \\
\hline TOTAL & 7,474 & 39.2 & & & & & & \\
\hline \multicolumn{9}{|c|}{ Inflammation-related Multimorbidity } \\
\hline Physical Only & 3,676 & 39.5 & 0.99 & {$[0.92,1.06]$} & & 0.86 & {$[0.79,0.93]$} & $* * *$ \\
\hline Mental Only & 507 & 39.5 & 0.99 & {$[0.87,1.12]$} & & 0.89 & {$[0.78,1.02]$} & \\
\hline Both & 1,406 & 37.7 & 0.91 & {$[0.84,1.00]$} & $*$ & 0.74 & {$[0.67,0.82]$} & $* * *$ \\
\hline None & 1,885 & 39.8 & & & & & & \\
\hline \multicolumn{9}{|c|}{ Inhaled Corticosteroids } \\
\hline TOTAL & 8,260 & 43.3 & & & & & & \\
\hline \multicolumn{9}{|c|}{ Inflammation-related Multimorbidity } \\
\hline Physical Only & 4,043 & 43.4 & 1.07 & {$[0.99,1.14]$} & & 0.81 & {$[0.75,0.88]$} & $* * *$ \\
\hline Mental Only & 567 & 44.2 & 1.10 & {$[0.97,1.24]$} & & 0.93 & {$[0.82,1.07]$} & \\
\hline Both & 1,666 & 44.6 & 1.12 & {$[1.03,1.22]$} & $*$ & 0.78 & {$[0.71,0.86]$} & $* * *$ \\
\hline None & 1,984 & 41.9 & & & & & & \\
\hline
\end{tabular}

Note: Based on 19,060 Medicaid Beneficiaries with newly diagnosed COPD obtained from Medicaid Analytic eXtract files observed during 2005-2008. Asterisks represent significant group differences in likelihood of respective COPD medication use by inflammation-related multimorbidity compared to the reference group (None) obtained from Unadjusted and Adjusted Logistic Regression Analyses.

Adjusted Logistic Regression Analyses controlled for cohort year, sex, race, age, state, poverty eligibility, number of other clinical conditions, serious mental illness, alcohol abuse, substance abuse, tobacco use, polypharmacy, county level variables including: above high school education density (quartiles), unemployment density (quartiles), poverty density (quartiles), metro status, primary care shortage area, mental health shortage area, primary care provider density (quartiles), hospital beds density (quartiles), psychiatric hospital, pulmonologist density and cardiologist density.

OR: Odds Ratio; AOR: Adjusted Odds Ratio; CI: Confidence Interval; Sig: Significance

$* * * \mathrm{p}<.001 ; * * .001 \leq \mathrm{p}<.01 ; * .01 \leq \mathrm{p}<.05$ 


\begin{tabular}{|c|c|c|c|c|c|}
\hline \multicolumn{6}{|c|}{$\begin{array}{c}\text { Table } 2 \\
\text { Number, Percentage, Adjusted Odds Ratio and 95\% Confidence Interval from Multinomial } \\
\text { Logistic Regression on Type of Bronchodilator Use } \\
\text { among Medicaid Beneficiaries with Newly-Diagnosed COPD } \\
\text { Medicaid Analytic Extract, 2005-2008 }\end{array}$} \\
\hline \multicolumn{6}{|c|}{ Short Acting Bronchodilators Only } \\
\hline & $\mathbf{N}$ & Row\% & AOR & $95 \% \mathrm{CI}$ & Sig \\
\hline TOTAL & 5,836 & 30.6 & & & \\
\hline \multicolumn{6}{|c|}{ Inflammation-related Multimorbidity } \\
\hline Physical Only & 2,870 & 30.8 & 0.88 & {$[0.79,0.97]$} & $* *$ \\
\hline Mental Only & 397 & 30.9 & 1.01 & {$[0.86,1.20]$} & \\
\hline Both & 1,207 & 32.3 & 0.80 & {$[0.71,0.91]$} & $* * *$ \\
\hline None & 1,362 & 28.8 & & & \\
\hline \multicolumn{6}{|c|}{ Long Acting Bronchodilators Only } \\
\hline TOTAL & 1,133 & 5.9 & & & \\
\hline \multicolumn{6}{|c|}{ Inflammation-related Multimorbidity } \\
\hline Physical Only & 599 & 6.4 & 1.01 & {$[0.85,1.20]$} & \\
\hline Mental Only & 54 & 4.2 & 0.77 & {$[0.56,1.06]$} & \\
\hline Both & 206 & 5.5 & 0.85 & {$[0.68,1.06]$} & \\
\hline None & 274 & 5.8 & & & \\
\hline \multicolumn{6}{|c|}{ Both Bronchodilators } \\
\hline TOTAL & 6,341 & 33.3 & & & \\
\hline \multicolumn{6}{|c|}{ Inflammation-related Multimorbidity } \\
\hline Physical Only & 3,077 & 33.1 & 0.77 & {$[0.70,0.85]$} & $* * *$ \\
\hline Mental Only & 453 & 35.3 & 0.92 & {$[0.78,1.09]$} & \\
\hline Both & 1,200 & 32.1 & 0.63 & {$[0.56,0.72]$} & $* * *$ \\
\hline None & 1,611 & 34.0 & & & \\
\hline
\end{tabular}

Note: Based on 19,060 Medicaid Beneficiaries with newly diagnosed COPD obtained from Medicaid Analytic eXtract files observed during 2005-2008. Asterisks represent significant group differences in likelihood of bronchodilator use by inflammation-related multimorbidity compared to the reference group (None) obtained from Multinomial Logistic Regression Analyses.

Adjusted Logistic Regression Analyses controlled for cohort year, sex, race, age, state, poverty eligibility, number of other clinical conditions, serious mental illness, alcohol abuse, substance abuse, tobacco use, polypharmacy, county level variables including: above high school education density (quartiles), unemployment density (quartiles), poverty density (quartiles), metro status, primary care shortage area, mental health shortage area, primary care provider density (quartiles), hospital beds density (quartiles), psychiatric hospital, pulmonologist density and cardiologist density.

OR: Odds Ratio; $\quad$ AOR: Adjusted Odds Ratio; CI: Confidence Interval; Sig: Significance

$* * * \mathrm{p}<.001 ; * * .001 \leq \mathrm{p}<.01 ; * .01 \leq \mathrm{p}<.05$ 


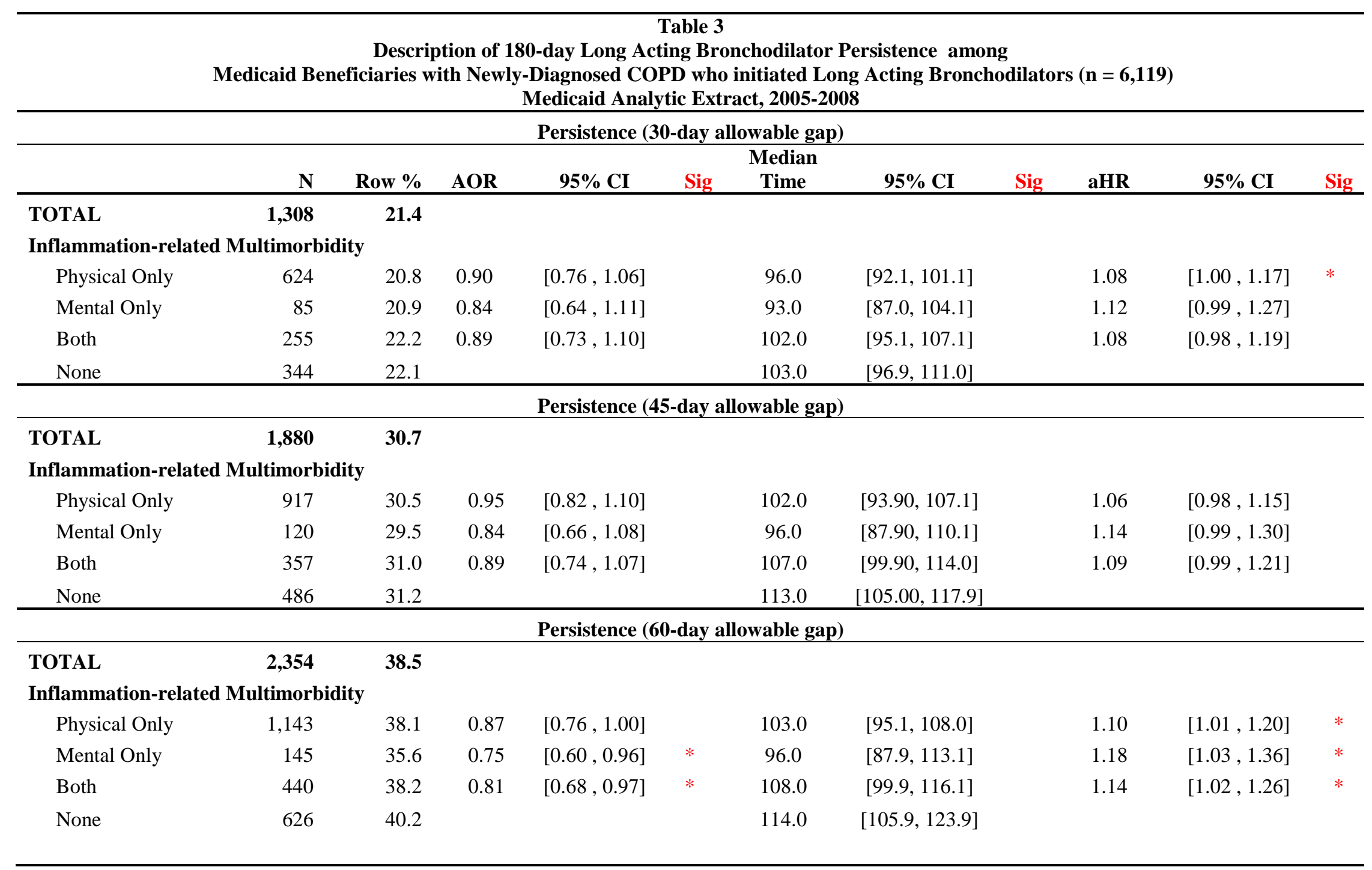

Note: Based on 6,119 Medicaid Beneficiaries with newly diagnosed COPD who initiated long-acting bronchodilators, obtained from Medicaid Analytic eXtract files observed during 2005-2008. Asterisks represent significant group differences in likelihood of 180-days persistence, median time to discontinuation (measured in days) and hazards of discontinuation. 
Adjusted Logistic Regression Analyses controlled for cohort year, sex, race, age, state, poverty eligibility, number of other clinical conditions, serious mental illness, alcohol abuse, substance abuse, tobacco use, polypharmacy, county level variables including: above high school education density (quartiles), unemployment density (quartiles), poverty density (quartiles), metro status, primary care shortage area, mental health shortage area, primary care provider density (quartiles), hospital beds density (quartiles), psychiatric hospital, pulmonologist density and cardiologist density.

OR: Odds Ratio; AOR: Adjusted Odds Ratio; aHR: Adjusted Hazards Ratio; CI: Confidence Interval; Sig: Significance

$* * * \mathrm{p}<.001 ; * * .001 \leq \mathrm{p}<.01 ; * .01 \leq \mathrm{p}<.05$ 
Table 4

Number, Percentage, Adjusted Odds Ratio and 95\% Confidence Interval from Logistic Regressions on COPD-specific and COPD-related Healthcare Utilization among Medicaid Beneficiaries with Newly-Diagnosed COPD Medicaid Analytic Extract, 2005-2008

\begin{tabular}{|c|c|c|c|c|c|}
\hline \multicolumn{6}{|c|}{ COPD-specific Hospitalizations } \\
\hline & $\mathbf{N}$ & Row \% & AOR & $95 \% \mathrm{CI}$ & Sig \\
\hline TOTAL & 998 & 5.2 & & & \\
\hline \multicolumn{6}{|c|}{ Inflammation-related Multimorbidity } \\
\hline Physical Only & 478 & 5.1 & 0.81 & {$[0.68,0.96]$} & $*$ \\
\hline Mental Only & 72 & 5.6 & 1.01 & {$[0.77,1.33]$} & \\
\hline Both & 205 & 5.5 & 0.80 & {$[0.65,0.99]$} & $*$ \\
\hline None & 243 & 5.1 & & & \\
\hline \multicolumn{6}{|c|}{ COPD-specific Emergency Room visits } \\
\hline TOTAL & 2,824 & 14.8 & & & \\
\hline \multicolumn{6}{|c|}{ Inflammation-related Multimorbidity } \\
\hline Physical Only & 1,322 & 14.2 & 0.83 & {$[0.75,0.93]$} & $* * *$ \\
\hline Mental Only & 198 & 15.4 & 0.91 & {$[0.76,1.09]$} & \\
\hline Both & 591 & 15.8 & 0.85 & {$[0.74,0.97]$} & $*$ \\
\hline None & 713 & 15.1 & & & \\
\hline \multicolumn{6}{|c|}{ COPD-specific Outpatient Visits } \\
\hline TOTAL & 7,200 & 43.7 & & & \\
\hline \multicolumn{6}{|c|}{ Inflammation-related Multimorbidity } \\
\hline Physical Only & 3,462 & 43.7 & 1.02 & {$[0.94,1.11]$} & \\
\hline Mental Only & 499 & 43.8 & 1.02 & {$[0.89,1.17]$} & \\
\hline Both & 1,448 & 44.9 & 1.02 & {$[0.92,1.13]$} & \\
\hline None & 1,791 & 42.7 & & & \\
\hline \multicolumn{6}{|c|}{ COPD-related Hospitalizations } \\
\hline TOTAL & 3,052 & 16.0 & & & \\
\hline \multicolumn{6}{|c|}{ Inflammation-related Multimorbidity } \\
\hline Physical Only & 1,486 & 16.0 & 1.12 & {$[1.00,1.25]$} & \\
\hline Mental Only & 198 & 15.4 & 1.06 & {$[0.89,1.28]$} & \\
\hline Both & 779 & 20.9 & 1.26 & {$[1.10,1.44]$} & $* * *$ \\
\hline None & 589 & 12.4 & & & \\
\hline \multicolumn{6}{|c|}{ COPD-related Emergency Room visits } \\
\hline TOTAL & 4,092 & 21.4 & & & \\
\hline \multicolumn{6}{|c|}{ Inflammation-related Multimorbidity } \\
\hline Physical Only & 1,943 & 20.9 & 0.87 & {$[0.79,0.95]$} & $* *$ \\
\hline Mental Only & 293 & 22.8 & 1.02 & {$[0.87,1.19]$} & \\
\hline Both & 891 & 23.9 & 0.92 & {$[0.82,1.03]$} & \\
\hline None & 965 & 20.4 & & & \\
\hline
\end{tabular}


Note: Based on 19,060 Medicaid Beneficiaries with newly diagnosed COPD obtained from Medicaid Analytic eXtract files observed during 2005-2008. Asterisks represent significant group differences in likelihood of respective COPD-specific healthcare utilization by inflammation-related multimorbidity compared to the reference group (None) obtained from Adjusted Logistic Regression Analyses.

Adjusted Logistic Regression Analyses controlled for cohort year, sex, race, age, state, poverty eligibility, number of other clinical conditions, serious mental illness, alcohol abuse, substance abuse, tobacco use, polypharmacy, county level variables including: above high school education density (quartiles), unemployment density (quartiles), poverty density (quartiles), metro status, primary care shortage area, mental health shortage area, primary care provider density (quartiles), hospital beds density (quartiles), psychiatric hospital, pulmonologist density and cardiologist density.

OR: Odds Ratio; $\quad$ AOR: Adjusted Odds Ratio; CI: Confidence Interval; Sig: Significance

$* * * \mathrm{p}<.001 ; * * .001 \leq \mathrm{p}<.01 ; * .01 \leq \mathrm{p}<.05$ 


\begin{tabular}{|c|c|c|c|c|c|c|c|c|}
\hline \multicolumn{9}{|c|}{$\begin{array}{r}\text { Table } 5 \\
\text { Mean, Standard Deviation and Parameter Estimates from } \\
\text { Total and Type of COPD-specific Healthcar } \\
\text { among Medicaid Beneficiaries with Newly Diagn } \\
\text { Medicaid Analytic Extract, 2005-20 }\end{array}$} \\
\hline \multicolumn{9}{|c|}{$\begin{array}{c}\text { Total COPD-expenditures } \\
\end{array}$} \\
\hline & & & \multicolumn{3}{|c|}{ Unadjusted Model } & \multicolumn{3}{|c|}{ Fully-adjusted Model } \\
\hline & Mean (SD) & Median & beta & SE & Sig & beta & SE & Sig \\
\hline \multicolumn{9}{|l|}{ TOTAL } \\
\hline Intercept & & & 2872.0 & 180.1 & $* * *$ & 7.47 & 0.19 & $* * *$ \\
\hline \multicolumn{9}{|c|}{ Inflammation-related Multimorbidity } \\
\hline Physical Only & $3481.7(14115.1)$ & 312.7 & 609.8 & 221.2 & $* *$ & -0.07 & 0.03 & * \\
\hline Mental Only & $2588.3(8163.0)$ & 340.4 & -283.7 & 390.1 & & -0.20 & 0.05 & $* * *$ \\
\hline Both & $3317.6(9517.5)$ & 390.9 & 445.6 & 271.3 & & -0.26 & 0.04 & $* * *$ \\
\hline None & $2872.0(11710.8)$ & 314.0 & & & & & & \\
\hline \multicolumn{9}{|c|}{ COPD-specific Inpatient Expenditures (Among Inpatient Users; $n=998$ ) } \\
\hline \multicolumn{9}{|l|}{ TOTAL } \\
\hline Intercept & & & 8418.11 & 739.50 & $* * *$ & 7.98 & 0.39 & $* * *$ \\
\hline \multicolumn{9}{|c|}{ Inflammation-related Multimorbidity } \\
\hline Physical Only & $7180.7(13391.5)$ & 4340.0 & -1237.40 & 908.22 & & -0.18 & 0.07 & $* *$ \\
\hline Mental Only & $5888.8(7417.5)$ & 4472.0 & -2529.30 & 1546.77 & & -0.37 & 0.11 & $* * *$ \\
\hline Both & $6373.8(6093.9)$ & 4200.0 & -2044.30 & 1093.20 & & -0.26 & 0.09 & $* *$ \\
\hline None & $8418.1(12037.3)$ & 4876.0 & & & & & & \\
\hline \multicolumn{9}{|c|}{ COPD-specific Outpatient Expenditures (Among Outpatient Users; $n=16,480$ ) } \\
\hline \multicolumn{9}{|l|}{ TOTAL } \\
\hline Intercept & & & 440.28 & 24.10 & $* * *$ & 6.76 & 0.15 & $* * *$ \\
\hline \multicolumn{9}{|c|}{ Inflammation-related Multimorbidity } \\
\hline Physical Only & $425.4(1376.6)$ & 133.4 & -14.83 & 29.80 & & -0.09 & 0.03 & $* * *$ \\
\hline Mental Only & $372.3(1362.0)$ & 142.7 & -67.98 & 52.17 & & -0.19 & 0.04 & $* * *$ \\
\hline Both & $421.5(1635.4)$ & 147.0 & -18.77 & 36.55 & & -0.15 & 0.03 & $* * *$ \\
\hline None & $440.3(1852.4)$ & 141.6 & & & & & & \\
\hline
\end{tabular}

Note: Based on data of 19,060 Medicaid Beneficiaries with newly diagnosed COPD obtained from Medicaid Analytic eXtract files observed during 2005-2008. Asterisks represent significant group differences in expenditures by inflammation-related multimorbidity compared to the reference group (None) obtained from Unadjusted and Adjusted Generalized Linear Model with Log-Link and Gamma Distribution.

Adjusted Logistic Regression Analyses controlled for cohort year, sex, race, age, state, poverty eligibility, number of other clinical conditions, serious mental illness, alcohol abuse, substance abuse, tobacco use, polypharmacy, county level variables including: above high school education (quartiles), unemployment density (quartiles), poverty density (quartiles), metro status, primary and, mental health shortage area, primary care provider density (quartiles), hospital beds density (quartiles), psychiatric hospital, pulmonologist and cardiologist density.

SD: Standard Deviation; Sig: Significance; SE: Standard Errors

$* * * \mathrm{p}<.001 ; * * .001 \leq \mathrm{p}<.01 ; * .01 \leq \mathrm{p}<.05$ 


\section{CHAPTER 3}




\section{CHAPTER 3: REAL-WORLD STUDY OF CLINICAL AND ECONOMIC OUTCOMES OF STATIN MEDICATIONS AMONG MEDICAID BENEFICIARIES WITH NEWLY- DIAGNOSED COPD}

\section{INTRODUCTION}

Currently the treatment for Chronic Obstructive Pulmonary Disease (COPD) focuses on reducing the frequency of acute exacerbations and relieving the symptoms of COPD such as shortness of breath (Global strategy for the diagnosis, management, and prevention on chronic obstructive pulmonary disease: 2013 update.2013) through acute and maintenance pharmacologic therapies as well as pulmonary rehabilitation. To date, no therapeutic options, which alter the prognosis of disease also known as disease modifying drugs for COPD treatment, have been established. However, new insights into the pathophysiology of the disease have opened up opportunities to use therapeutic regimens that are currently available for circulatory diseases to treat COPD. (M. Cazzola, Ciaprini, Page, \& Matera, 2007; Fabbri \& Rabe, 2007)

The main pathophysiology of COPD revolves around airways inflammation which limits normal flow of air through the lungs. (Górska, Maskey-Warzechowska, \& Krenke, 2010; Rabe et al., 2007) Inflammation in COPD can be caused because of lung exposure to risk factors such as smoking and air pollution (indoor, outdoor and/or occupational).(Global strategy for the diagnosis, management, and prevention on chronic obstructive pulmonary disease: 2013 update.2013) As per the conventional theories inflammation should be limited only to the pulmonary system. However, evidence has been growing regarding the extra-pulmonary inflammation also known as systemic inflammation (Matera, Calzetta, Rinaldi, \& Cazzola, 2012; Nussbaumer-Ochsner \& Rabe, 2011; Vogelmeier \& Wouters, 2011) and COPD is also being considered as a disease associated with systemic inflammation (P. J. Barnes \& Celli, 2009; Fabbri \& Rabe, 2007; Sevenoaks \& Stockley, 2006). Systemic inflammation in COPD can be 
due to the 'spillover' effect of high concentration of inflammatory mediators in the lungs (P. J. Barnes, 2010) or due to the high prevalence of inflammation-related multimorbidity among individuals with COPD. (P. J. Barnes \& Celli, 2009) For example, highly prevalent chronic conditions such as cardiovascular diseases, musculoskeletal disorders, depression and diabetes mellitus have been linked to systemic inflammation outside the lungs among individuals with COPD. (P. J. Barnes, 2010; M. Cazzola, Bettoncelli, Sessa, Cricelli, \& Biscione, 2010; M. Cazzola et al., 2012) Recently, it has been documented that individuals with COPD are more likely to have certain chronic conditions with common inflammatory pathway as compared to individuals without COPD. These conditions include depression (20.6\% vs. $12.5 \%)$, coronary heart disease ( $12.7 \%$ vs. $6.1 \%)$, congestive heart failure ( $12.1 \%$ vs. $3.9 \%)$, and stroke (8.9\% vs. 4.6\%). (Schnell et al., 2012) Unfortunately, the current acute and maintenance pharmacologic therapies for COPD management do not specifically target the systemic inflammation. (M. Cazzola et al., 2007; Matera et al., 2012) Therefore, it is important to evaluate the effects of other inflammation reducing medications such as 3-hydroxy 3-methylglutaryl coenzyme A reductase inhibitors (HMG CoA reductase inhibitors) commonly known as statins, that may alter the disease prognosis among individuals with COPD.

\section{Inability of Current Medications to Treat Systemic Inflammation}

Medications commonly used for acute and maintenance COPD management include bronchodilators and corticosteroids. (Global strategy for the diagnosis, management, and prevention on chronic obstructive pulmonary disease: 2013 update.2013) Indirect evidence exists that these medications are potent in treating pulmonary inflammation and thus may be helpful in relieving symptoms of COPD. Some experiments on animals have provided evidence that currently available inhalation therapies can reduce levels of systemic inflammatory mediators. (Suda et al., 2011) However, these results were not consistent with those observed in 
a large multicenter randomized controlled trial. (Sin et al., 2008) Findings from this trial revealed that the combination of inhaled corticosteroid and long acting beta-2-agonist reduced pulmonary inflammation leading to improvements in COPD symptoms but these medications did not reduce systemic inflammation. It has also been suggested that inhalation therapies may have limited systemic effects due to their mode of administration. (M. Cazzola et al., 2007; Vogelmeier \& Wouters, 2011) In this context, researchers have been exploring the potential utility of other medications that may lead to reduction in systemic inflammation among individuals with COPD.

\section{Statins and Anti-Inflammatory Properties}

Statins, a class of cholesterol lowering drugs have demonstrated their effectiveness in prevention and management of cardiovascular diseases. (Baigent et al., 2005) Based on the review of in vitro as well as in vivo studies Schönbeck and Libby (Schonbeck \& Libby, 2004) concluded that statins also have anti-inflammatory functions. In a study conducted by Lee et al (2005), it was found that the inflammation caused by smoking in lungs of rats was alleviated by lipophilic statin, specifically simvastatin. (J. Lee et al., 2005) In humans, researchers using a randomized controlled trial have shown that pravastatin (hydrophilic statin) use significantly reduced levels of inflammatory biomarkers. (T. Lee, Lin, \& Chang, 2008) The evidence has also been supported by preclinical models that have revealed the pleiotropic/anti-inflammatory therapeutic benefits attributable to statins. (Takahashi et al., 2008) These facts derived from several mechanistic studies suggest that statin use has emerged as a novel therapeutic intervention for individuals with COPD. (Walsh, 2008)

\section{Statin use and Improved Outcomes among individuals with COPD}

Since early 2000, evidence of beneficial impact of statins on outcomes among individuals with COPD has become available. One randomized trial (T. Lee et al., 2008) and some observational studies have been conducted (Blamoun et al., 2008; Huang et al., 2011; Ishida et 
al., 2007; Keddissi et al., 2007; Lawes et al., 2012) to examine the role of statins in COPD management. Most of these studies have provided evidence of beneficial effects of statins in improving lung function, (Keddissi et al., 2007), exercise time as a measure of health status (T. Lee et al., 2008) and reducing COPD exacerbations (Blamoun et al., 2008) and COPD-related hospitalizations (Mancini Huang), all-cause (Frost, Petersen, Tollestrup, \& Skipper, 2007; Lawes et al., 2012; Lawes et al., 2012; Mancini et al., 2006; Søyseth et al., 2007; Søyseth, Brekke, Smith, \& Omland, 2007)and COPD-specific mortality. (Frost et al., 2007)

In retrospective cohort study using Veteran's Affairs data among individuals with COPD, investigators have shown that statin use (including simvastatin, lovastatin, atorvastatin and fluvastatin) was associated with improved lung function as measured by slower decline pulmonary function compared no statin use. (Keddissi et al., 2007) Similarly, investigators of a randomized controlled trial among individuals with COPD found that pravastatin use for 6 months significantly increased exercise time from baseline as compared to placebo group suggesting improvements in health status. (T. Lee et al., 2008)

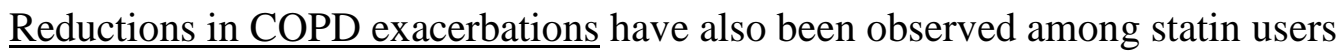
compared to non-users. In a study based on hospitalized COPD patients in a regional medical center $(n=185)$, investigators reported that individuals not receiving statins were approximately twice as likely to experience COPD exacerbation (Adjusted Odds Ratio (AOR): 2.35; 95\% CI: 1.01-5.50) and 10 times as likely to require intubation for exacerbation (AOR: 10.36; 95\% CI: 2.77-38.76) compared to non-users of statins. (Blamoun et al., 2008) This study was limited by its small sample size, inability to adjust for a variety of risk factors which may affect the relationship between statin use and COPD-related health outcomes such as co-morbidities. Similarly, a study using Veterans Affairs hospital patients $(n=418)$ with COPD revealed that 
individuals without statin use reported significantly higher mean of COPD exacerbations as compared to those with statin use $(0.17 \pm 0.31$ vs. $0.11 \pm 0.26 ; p=0.01)$. (Keddissi et al., 2007)

Likelihood of COPD-specific hospitalizations has been shown to be lower among users of statins compared to non-users of statins. Findings from a time-matched nested case-control study on effects of statin use on elderly individuals COPD, indicated that compared to no statin use, any statin use was associated with reduced likelihood of COPD related hospitalization irrespective of their cardiovascular risk (HI CV risk- RR: 0.71; 95\% CI: 0.56-0.91 and low CV risk - RR: 0.71; 95\% CI: 0.64-0.77). (Mancini et al., 2006)These findings were consistent with those obtained from nationwide cohort study in Taiwan. (Huang et al., 2011)This study reported that among 6,252 newly diagnosed individuals with COPD statin use significantly decreased hazards of COPD-related hospitalization (HR: 0.66; 95\% CI: 0.60-0.74) compared to no statin use.

\section{Need for the Study}

COPD is generally considered as an irreversible disease with current medications focusing towards improving the symptoms associated with COPD. (GOLD, young, matera) Based on the healthy people goals for 2020 it has been suggested that improvements in COPD management should include reducing hospitalizations associated with COPD. (Healthy people 2020: Goals for respiratory diseases.2013) Moreover, hospitalizations for COPD are considered to be preventable and thus are used as a metric to measure quality of care. (Quality indicator user guide: Prevention quality indicators (PQI) composite measures version 4.3.2011) Current, COPD medications have not been shown to alter the prognosis of disease mainly because of their inability to target and treat systemic inflammation. The healthy people 2020 goals for COPD specifically mention the need for developing novel treatments for COPD which can improve the overall prognosis of disease. (Healthy people 2020: Goals for respiratory diseases.2013) 
Therefore, there is need to generate evidence on novel therapies that may positively impact systemic inflammation and therefore modify the course of COPD.

In this context, Statins, due to their anti-inflammatory properties are being evaluated as a novel therapy to manage COPD. (Young, Hopkins, \& Eaton, 2009) However, evidence regarding health outcomes such as healthcare utilization and expenditures associated with statin therapy among individuals with COPD is limited. In addition, the currently available studies have several limitations which restrict our ability to better understand the relationship between statin therapy and COPD-specific health outcomes. First, the study conducted to assess the impact of statin use among veterans with COPD had limited sample size $(\mathrm{n}=418)$ and only represented hospitalized patients who were either former or current smokers. (Keddissi et al., 2007) The other studies that evaluated the use of statins for improving COPD-specific outcomes had several shortcomings such as non-generalizability to US population (Huang et al., 2011; Mancini et al., 2006) and conducted on individuals with COPD and cardiovascular risk. (Mancini et al., 2006)

COPD is a disease associated with systemic inflammation and therefore has been linked with high rates of inflammation-related multimorbidity. Researchers using NHANES data have reported that 96\% of individuals with COPD have at least one co-occurring conditions. (Schnell et al., 2012) Therefore, while studying the effect of statins on healthcare utilization among individuals with COPD and generating evidence base on the novel therapies for individuals with COPD, it is important to understand whether statin therapy can improve outcomes high-risk and more vulnerable population i.e. those with inflammation-related multimorbidity.

This study is highly timely and innovative in providing important evidence on the association between novel approaches (statin therapy) for COPD management and COPDspecific outcomes. In addition, randomized clinical trials routinely exclude those with 
multimorbidity and therefore using data from real-world settings is required to generate generalizable evidence for the management of individuals with multimorbidity. Our study is hypothesis generating and will provide the necessary foundation for future research and perhaps randomized clinical trials evaluating the effectiveness of statin therapy among individuals with COPD.

An important contribution of our study is providing knowledge on the association between statin therapy and COPD-specific outcomes among young adults, specifically Medicaid beneficiaries. Enrollees in the Medicaid plans comprise of high-risk vulnerable population that have high rates of multimorbidity. In fact, Medicaid beneficiaries with COPD have very high rates of inflammation-related multimorbidity. Researchers using the Maryland Medicaid database have reported that beneficiaries with COPD had higher prevalence of diabetes mellitus $(27.7 \%)$, congestive heart failure $(17.9 \%)$ and cerebrovascular disease $(10.7 \%)$. (Lin, Shaya, \& Scharf, 2010)Therefore, proving the benefits of statins among high-risk and vulnerable population of Medicaid beneficiaries with COPD will strengthen the evidence in the area of COPD management. 


\section{CONCEPTUAL FRAMEWORK}

In this study we used an adaption of Economic Clinical and Humanistic Outcomes Model (ECHO). The ECHO model is described in the Figure below.

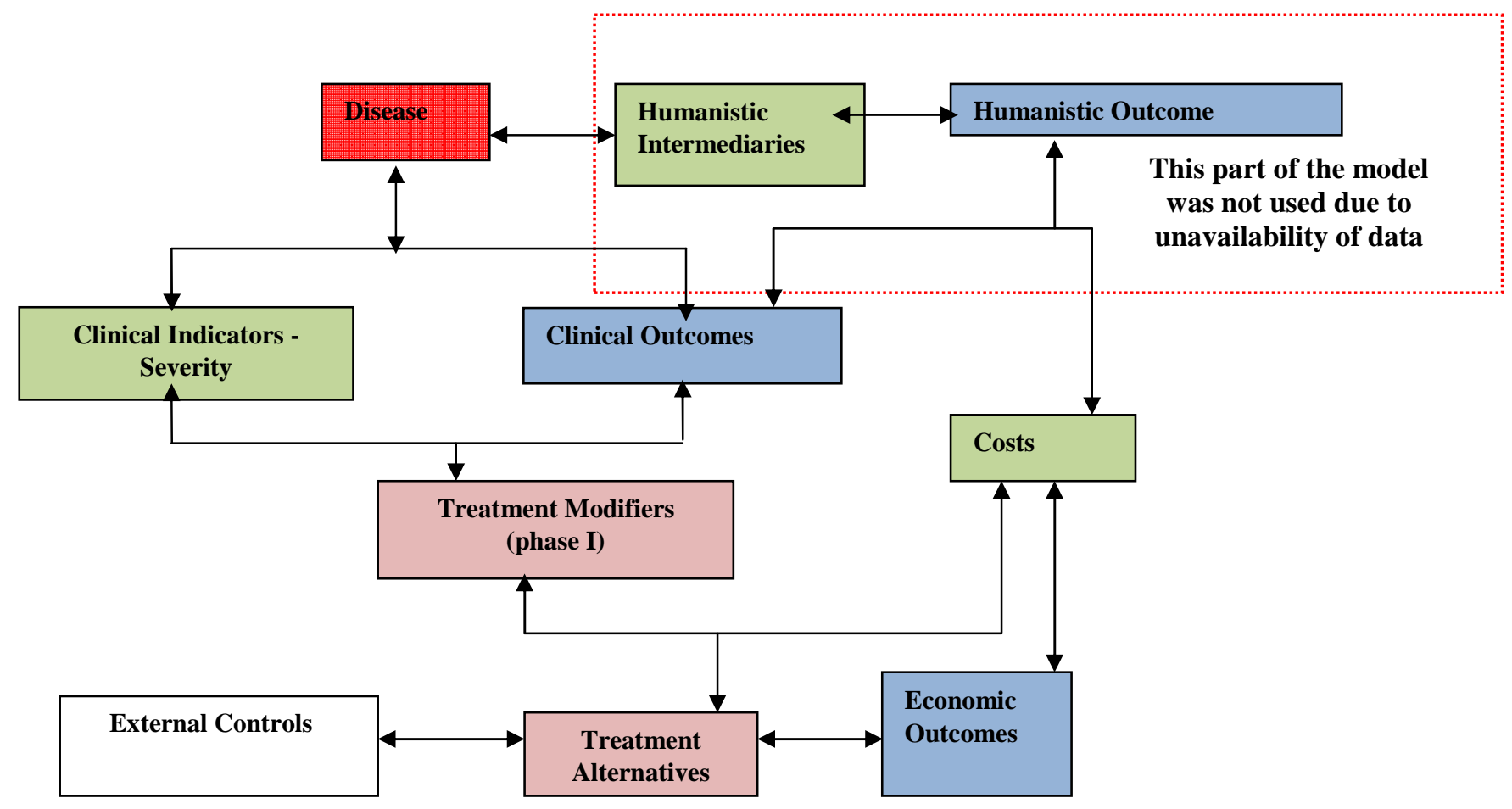

Figure 1: Description of ECHO Model

ECHO Model: ECHO model identifies, various pathways which affects the economic, clinical and humanistic outcomes

The theoretical framework provided in the ECHO model has been proposed by Kozma et al (Kozma, Reeder, \& Schulz, 1993) in 1993 and adapted in protocol development guideline for observational comparative effectiveness research by Agency for Healthcare Research and Quality. The model describes pathways which are associated with relationship between treatments and health outcomes. This model also depicts that that the choice of treatment alternatives is affected by several external controls. These external controls include nonmodifiable factors such as race, gender, age and modifiable factors such as personal health 
practices such as tobacco, alcohol and drug use. The model indicates that disease (COPD) affects clinical indicators and clinical outcomes (such as disease biomarkers, hospitalizations and others). On the other hand presence of external control affects treatment choices. Once the treatment is provided through various pathways the treatment improves clinical outcomes and can lead to reduction in healthcare expenditures (i.e. economic outcomes).

\section{METHODS}

\section{Study design}

Retrospective longitudinal dynamic cohort design using data from administrative claims of Medicaid beneficiaries was utilized. Multiple years of Medicaid Analytic eXtract (MAX) files (2005-2008) provided by the Centers for Medicare and Medicaid Services (CMS) were used to construct the study cohort. Individuals with COPD were identified between 01/01/2006 and 12/31/2007 (Index date Identification Period). Receipt of statin medication and other baseline characteristics were identified in the baseline period which was defined as 12 months before the index date. One year period after index date was considered as the follow-up period. This period was used to assess COPD-specific healthcare utilization such as COPD-specific hospitalizations, COPD-specific ER visits and COPD-specific outpatient visits. Total healthcare and COPDspecific healthcare expenditures were also identified during this period.
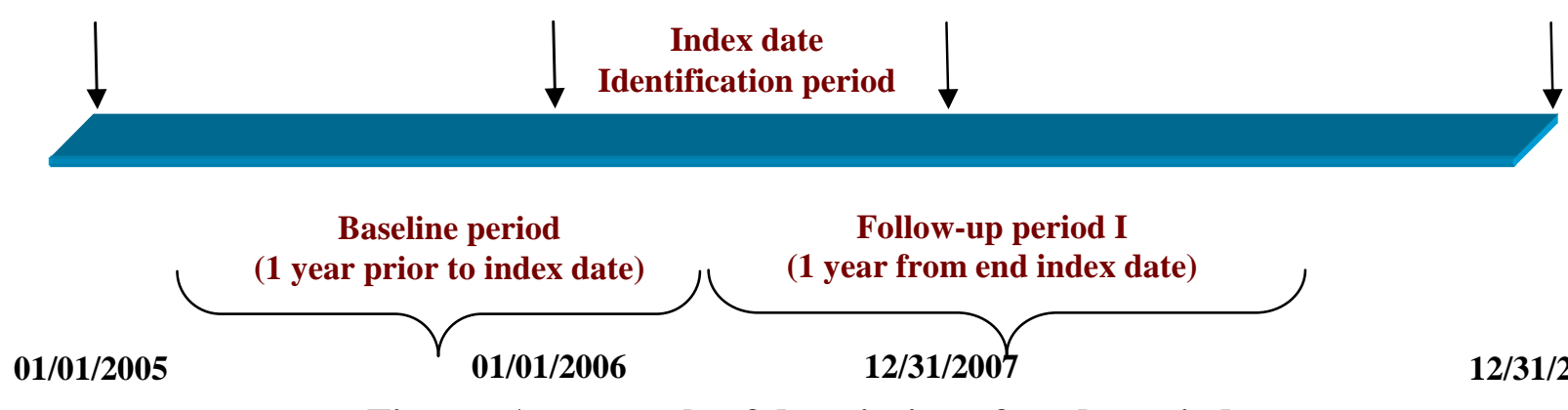

Figure: An example of description of study periods 


\section{Data Source}

\section{Medicaid Analytic eXtract (MAX) (2005-2008)}

Medicaid analytic extract (MAX) files prepared and produced by the Centers for Medicare and Medicaid Services with the help of Research and Data Assistance Center (ResDAC) for performing research on healthcare utilization and outcomes among Medicaid beneficiaries were used for this study. The data for Medicaid beneficiaries is collected via Medicaid Statistical Information System (MSIS). States administering the Medicaid program report annual, person-level data for the Medicaid beneficiaries to the CMS through MSIS. These person level files provided information about beneficiaries' eligibility, their healthcare utilization and payment information. The quality of these files is maintained through series of initiatives taken by the CMS and ResDAC. (CMS, 2011; Hennessy, Leonard, Palumbo, Newcomb, \& Bilker, 2007) We received five separate files ("personal summary (PS)", "inpatient claims (IP)", "other therapy claims (OT)", "prescription drug claims (RX)" and "long-term care claims (LT)") from CMS. For the purposed of this study we linked PS, IP, OT, RX files based on beneficiaries' unique identification number. The PS files included information on eligibility, demographics, managed care enrollment, and utilization summary. The other three files provided information on fee-for-service claims data for services used. For example, the RX file included information regarding utilization of prescription drugs by Medicaid beneficiaries. This information includes, date of prescription filled, days of supply, national drug code (NDC) which were used to identify the receipt of statin therapy. We used data for beneficiaries residing in the following states: New York (NY), Texas (TX), Illinois (IL), and California (CA). The primary reason behind using this data was to obtain programs with lower Medicaid-managed care penetration rates so that more utilization characteristics would be captured. This provided us the ability to obtain maximum number of beneficiaries with fee-for-service enrollments. 


\section{Area Resource File (ARF):}

In addition to all the demographic characteristics and utilization variables obtained from the MAX files, we also determined each Medicaid beneficiary's contextual county level variables using the Area Resource File (ARF). ARF is a comprehensive county-level dataset compiled by the Health Resources Service Administration's Bureau of Health Professions. ARF contains more than 6000 variables providing information about nation's counties. The information contained within ARF includes type of health facilities in the various counties, number and type health professions, resource scarcity measures, health status, economic activity, health training programs, and socioeconomic and environmental characteristics. These files also include county codes and descriptors that allow its linkage with several secondary datasets such as MAX. The type of variables that were obtained from ARF included density of pulmonologist in beneficiary's residing county. We used the county codes and state information to link MAX files with 2008 ARF file. All the ARF variables were obtained from the baseline period.

\section{Study Population}

\section{Medicaid Beneficiaries with COPD}

Medicaid beneficiaries with diagnosed COPD were identified using MAX IP and OT files (physician office visits claims only). Baseline period were used to identify Medicaid Beneficiaries with COPD. Individuals with at least one inpatient visit or two 14 days apart outpatient visits (obtained using type of service codes) for COPD based on International Classification of Diseases, Ninth Revision, Clinical Modification (ICD-9-CM) codes for chronic bronchitis (491.xx), emphysema (492.xx), or unspecified chronic airway obstruction (496.xx) were considered to have diagnosed COPD. These diagnosis codes have been utilized in prior published research (codes 491.xx, 492.xx and 496.xx) to identify COPD and evaluate medical treatment and health outcomes among individuals with COPD. (Dalal, Shah, D'Souza, 
Chaudhari, \& Crater, 2012; Halpern et al., 2011; Make, Dutro, Paulose-Ram, Marton, \& Mapel, 2012) Indeed, sensitivity and specificity of using ICD-9-CM codes to identify patients with COPD has been established. (Cooke et al., 2011; A. S. Gershon et al., 2009) A study conducted by Gershon et al reported that identifying COPD using 1 or more ambulatory claims and/or 1 or more hospitalizations for COPD resulted in a sensitivity of $85.0 \%$ (95\% CI: 77.0 to 91.0 ) and a specificity of $78.4 \%$ (95\% CI: 73.6 to 82.7). However, we will be used 1 inpatient or 2 outpatient claims to identify individuals with COPD in order to increase the specificity of the algorithm.

Other inclusion criteria will included: a) 40-64 years (among young adults this age group is at highest risk of COPD); b) continuous eligibility during the baseline and follow-up period; c) no dual Medicaid/Medicare coverage (dual eligibility will represent high-cost and severe beneficiaries); d) enrolled in fee-for-service plans throughout the study observation period; e) Alive during the study observation period; and use of services (inpatient or outpatient).

\section{Dependent Variables}

\section{COPD-Specific Outcomes}

Any healthcare claim with primary diagnosis of COPD identified using ICD-9-CM codes were considered within COPD-specific outcomes. Following variables were identified as COPDspecific outcomes: a) COPD-specific hospitalizations (yes/no); b) COPD-specific emergency room visits (yes/no); obtained from and inpatient and outpatient claims; and c) COPD-specific outpatient visits (low and high) derived by categorizing the number of COPD-specific outpatient visits greater than or equal to median and less than the median value.

\section{Total and COPD-specific Healthcare Expenditures}


We also measured Total and COPD- specific inpatient and outpatient expenditures. COPD-specific expenditures were identified by identifying service claims with the primary diagnosis of COPD (inpatient and outpatient). Total expenditures were estimated by adding Medicaid payment per claim (inpatient, outpatient and prescription drugs) for each person. To account for medical services inflation, expenditures in 2006 and 2007 were converted to 2008 dollars using the annual consumer price index for medical care services available from the Bureau of Labor Statistics. (BLS, 2013)

\section{Key Independent Variable}

\section{Treatment Alternatives}

1. Statin Therapy (Yes/No)

Statin therapy was identified in the baseline period using National Drug Code (NDC). Any Medicaid beneficiary with at least one prescription of statin during the baseline period was considered as receiving statin therapy.

\section{Long-term statin therapy (Long-term/short-term/no statin therapy)}

Individuals with long-term statin therapy were differentiated from those with short-term statin therapy. Medicaid beneficiaries with newly-diagnosed COPD having $\geq 120$ days of statin supply during the baseline period were considered as receiving long-term therapy. Those with $<120$ days of statin supply were categorized as individuals with short-term statin therapy and those without any claim of statin were grouped under no statin therapy.

\section{Clinical Severity (Inflammation-related multimorbidity)}

We created indicator variables for the presence of arthritis, CVD, depression, diabetes and osteoporosis using the International Classification of Diagnosis $-9^{\text {th }}$ Revision- Clinical Modification (ICD-9-CM) codes. Any Medicaid beneficiary with COPD having greater than or equal to one inpatient or one outpatient visits for these conditions were considered as having the 
disease. In addition indicator variables will be created for inflammation-related physical conditions and inflammation-related mental condition. Any Medicaid beneficiary having either of the following conditions: arthritis, CVD, diabetes and osteoporosis were grouped within inflammation-related physical conditions. Similarly, any individual with COPD and having depression were considered as having inflammation-related mental condition.

\section{Operational definition:}

Medicaid beneficiaries included in our study population were further grouped into one of the four multimorbidity categories as mentioned below:

5) COPD + Inflammation-related Physical Conditions + Inflammation-related Mental Condition

6) COPD + Inflammation-related Physical Conditions

7) COPD + Inflammation-related Mental Condition

8) COPD only (without any chronic conditions).

To analyze the relationship between statin therapy, presence of inflammation-related multimorbidity and COPD-specific healthcare outcomes, we created a variable with four categories: a) statin therapy and inflammation-related multimorbidity; b) no statin therapy and inflammation-related multimorbidity; c) statin therapy and no inflammation-related multimorbidity and d) no statin therapy and no inflammation-related multimorbidity. This variable was entered in the final logistic regression analyses, which included adjustment for baseline characteristics. 


\section{Other Independent Variables (External Controls)}

When examining the impact of treatment alternatives on clinical and economic outcomes, external controls may bias the relationship. Therefore, we adjusted for these external controls in the regression analyses. These variables included year of diagnosis (2006 vs. 2007), demographic characteristics such as gender (women, men), race (White, African Americans, Others), age in years (40-49, 50-59, 60-64), poverty eligibility (yes, no), medical eligibility (yes, no), number of other clinical conditions (none, 1-3, 4-6, >6), serious mental illness (yes, no), alcohol abuse (yes, no), substance abuse (yes, no), tobacco use (yes, no) and polypharmacy ( $<10$ drug classes, $\geq 10$ drug classes). We additionally controlled for county-level characteristics obtained from the ARF. These variables included quartiles for density of above high school education, unemployment, poverty, primary care providers, and specialist care providers were created. Density of these county level characteristics was calculated by dividing total number of each characteristic by the total county population. This density was further converted into per 1000 people by multiplying 1000 with the density. Other ARF characteristics included pulmonologist density (high vs. low), cardiologist density (high vs. low), etc. In addition to these variables, there is a possibility of bias in relationship between statin therapy and COPD-specific outcomes due to state policy variations. Therefore, we adjusted for fixed effects for state variations using dummy variable for the states (CA, IL, NY, TX) in our analyses.

\section{Statistical Analyses}

\section{Inverse Probability of Treatment Weighting (IPTW) Technique}

In order to account for selection bias due to observed differences in baseline characteristics among individuals with and without statin therapy, Inverse Probability of Treatment Weighting (IPTW) technique was used. This method allows us to calculate weights that are essentially the probability of an individual receiving the treatment (i.e. exposure in a nonrandomized study) 
conditional on their observed stable covariates. Higher weights are assigned to individuals who are under-represented and vice-versa for individuals who are over represented. We adjusted for the probability weight that we obtained from the logistic/multinomial logistic regressions on statin therapy and duration of statin therapy respectively in order to control for the observed selection bias. This created a balance in terms of distribution of potential confounders across treatment levels.

\section{Bivariate Analyses}

Subgroup differences in statin therapy and duration of statin therapy were tested using Chi-square tests of independence. In addition, bivariate differences in COPD-specific healthcare outcomes between those with and without statin therapy were also tested using Chi-square tests of independence. F-tests were used to determine the relationship between statin therapy and continuous outcomes.

\section{$\underline{\text { Multivariate Analyses }}$}

We used logistic regressions and multinomial logistic regressions to examine the factors associated with any statin and long-term statin therapy respectively. Multivariable logistic regressions with and without IPTW adjustment was be used to determine the relationship between statin therapy and COPD-specific outcomes when the dependent variables are categorical (example: COPD -specific hospitalizations). For analyzing expenditure outcomes we used multivariable GLM with log-link function and gamma distribution. As many Medicaid beneficiaries did not have any COPD-specific healthcare expenditures, we used two-part models. The first part of the model predicted the probability of having any COPD-specific utilization and the second part of the model included GLM with log-link and gamma distribution on healthcare 
expenditures for beneficiaries who were users. All the analyses were conducted using SAS 9.3 (SAS Institute, Cary, NC).

\section{RESULTS}

After applying the eligibility criteria as shown in Figure 1, the analytic cohort included 19,060 Medicaid beneficiaries with newly-diagnosed COPD. Any statin therapy was observed in $30.3 \%(n=5,771)$ of the Medicaid beneficiaries, out of which three quarters of beneficiaries had long-term statin therapy defined as $\geq 120$ days statin medication supply.

\section{Factors associated with Statin Therapy}

Several demographic and clinical factors were associated with statin therapy and duration of statin therapy among Medicaid beneficiaries with newly-diagnosed COPD. Females (AOR: 0.92; 95\% CI: 0.86, 0.99), African Americans (AOR: 0.56; 95\% CI: 0.56, 0.67), younger adults in 40-49 years (AOR: 0.52, 95\% CI: 0.47, 0.57) and 50-59 years (AOR: 0.72, 95\% CI: 0.66, 0.79) were less likely to receive statin therapy compared to males, whites, and older adults (60 64 years). Beneficiaries with inflammation-related physical condition only (AOR: 3.6, 95\% CI: $2.85,3.50)$ and those with both physical and mental conditions (AOR: 2.75, 95\% CI: 2.44, 3.10) were approximately three times as likely to receive statins as compared to those with no multimorbidity. Medicaid beneficiaries with the presence of life-style risk factors such as alcohol abuse and substance abuse were less likely to receive statin therapy; whereas those with polypharmacy (AOR:3.82, 95\% CI: 3.52, 4.14) were more likely to receive statins. Similar results were observed from multinomial logistic regressions on duration of statin therapy (longterm/short-term vs. no statin therapy) among Medicaid beneficiaries with newly-diagnosed COPD. Detailed results from these analyses are presented in Table 1(a) and Table 1(b).

\section{Statin Therapy and COPD-specific Outcomes}


As shown in Table 2, those with statin therapy had significantly lower rates of COPDspecific hospitalizations ( $4.7 \%$ vs. $5.2 \%$; p<0.05), COPD-specific ER visits (13.4\% vs. $15.4 \%$; $\mathrm{p}<0.001)$ and COPD-specific outpatient visits $(41.4 \%$ vs. $44.7 \%$; $\mathrm{p}<0.001)$ compared to adults without statin therapy. Similarly, beneficiaries with any duration of statin therapy (long-term or short-term) had lower rates of COPD-specific healthcare utilization. The reduction in COPDspecific healthcare utilization was also observed even after controlling for baseline characteristics. Beneficiaries with statin therapy were $16 \%$ less likely to have COPD-specific hospitalization (AOR: 0.84 ; 95\% CI: $0.71,0.98), 11 \%$ less likely to have COPD-specific emergency room visit (AOR: 0.89; $95 \%$ CI: $0.81,0.99$ ) and $14 \%$ less likely to have COPDspecific outpatient visit (AOR: 0.86; 95\% CI: 0.80, 0.92). Analyses with duration of statintherapy revealed that only long-term statin therapy was associated with lower COPD-specific healthcare outcomes. Beneficiaries with long-term therapy of statin were less likely to have COPD-specific hospitalizations (AOR: 0.81, 95\% CI: 0.71, 0.99), COPD-specific emergency room visits (AOR: 0.86; 95\% CI: 0.77, 0.96) and COPD-specific outpatient visits (AOR: 0.84; 95\% CI: 0.78, 0.92).

In order to control for selection bias in observed variables, we conducted same regression analyses after weighting with Inverse Probability Treatment Weights (IPTW) obtained from logistic and multinomial logistic regressions on statin therapy and duration of statin therapy respectively. These treatment weights were adjusted for the number of individuals in each category of statin therapy and duration of statin therapy. The results from these analyses are presented in Table 3. Even after using IPTW technique, individuals with statin therapy were significantly less likely to have COPD-specific healthcare outcomes. In fact, the magnitude of reduction and significance strength enhanced after controlling for selection bias using IPTW 
technique. Moreover, after using IPTW technique short-term statin therapy also showed significant reduction in COPD-specific hospitalizations (AOR: 0.83; 95\% CI: 0.75, 0.91). However, association between short-term statin therapy and COPD-specific emergency room visits and COPD-specific outpatient visits remained insignificant. We also conducted sensitivity analyses using unadjusted IPTW technique (using weights that were not adjusted for number of individuals who received statins). The results from sensitivity analyses were similar to those observed with adjusted IPTW technique. (Data not represented in tabular form)

\section{Statin Therapy, Inflammation-related Multimorbidity and COPD-specific Healthcare Utilization}

An analysis of relationship between statin therapy and COPD-specific healthcare outcomes in presence of inflammation-related multimorbidity revealed that beneficiaries with inflammation-related multimorbidity and statin therapy were less likely to have COPD-specific specific healthcare outcomes as compared to those with inflammation-related multimorbidity and no statin therapy. For example, beneficiaries with inflammation-related multimorbidity and statin therapy were $18 \%$ less likely to have COPD-specific hospitalization (AOR: 0.82; 95\% CI: 0.70 , 0.97) as compared to those with inflammation-related multimorbidity and no statin therapy. Detailed results are presented in Table 4.

\section{Statin Therapy and Total and COPD-specific Healthcare Expenditures}

Findings from bivariate analyses on total and COPD-specific healthcare expenditures are reported in Table 5. For the ease of representation we have calculated ratio of means for overall and subgroup expenditures for selected characteristics. As shown in the table, individuals with statin therapy were not significantly different in average total healthcare expenditures than those without statin therapy $(\$ 36,215$ vs. $\$ 35,724)$. Similarly, there was no difference in COPDspecific outpatient expenditures between beneficiaries with or without statin therapy. However, 
average COPD-specific inpatient expenditures (among users of inpatient services) were significantly lower among those who received statin therapy when compared with adults who did not receive statin therapy $(\$ 6,593$ vs. $\$ 7,457 ; \mathrm{p}<0.05)$. Interestingly, among beneficiaries with inflammation-related physical condition statin therapy was associated with lower average COPD-specific expenditures $(\$ 6,446$ vs. $\$ 7,594 ; \mathrm{p}<0.05)$ and higher average COPD-specific outpatient expenditures ( $\$ 451$ vs. $\$ 409 ; \mathrm{p}<0.01)$

After controlling for baseline characteristics in the regression analyses with log-link function and gamma distribution (Table 6), it was found that statin therapy was not significantly associated with lower average COPD-specific inpatient and outpatient expenditures. Furthermore, long-term statin therapy was associated with higher average total healthcare expenditures $(\$ 9,394$ vs. $\$ 8,759 ; \beta=0.02 ; p<0.001)$, whereas short-term statin therapy was associated with lower $(\$ 7,902$ vs. $\$ 8,759 ; \beta=-0.10 ; \mathrm{p}<0.001)$ average total healthcare expenditures as compared to no statin therapy.

\section{DISCUSSION}

This retrospective observational study was conducted to evaluate the clinical and economic outcomes associated with statin therapy among individuals with newly diagnosed COPD in a real-world practice setting. Approximately $30 \%$ of Medicaid beneficiaries with newly-diagnosed COPD received statin medications during the baseline period. In this study, we found that even after controlling for baseline characteristics and observed selection bias using IPTW technique, beneficiaries with statin therapy were $24 \%$ less likely to have COPD-specific hospitalizations, $19 \%$ less likely to have COPD-specific ER and 14\% less likely to have COPDspecific outpatient visits among individuals with newly-diagnosed COPD. Our results are consistent with findings from a handful of studies that have evaluated effects of statins in terms 
of COPD-related hospitalizations and found beneficial effects. (Mancini et al, 2006; Huang et al, 2011) However, to the best of our knowledge this is the first study to examine the relationship between statin therapy and COPD-specific outcomes including both clinical and economic outcomes. Furthermore, this is the only study to assess the impact of duration of statin therapy among individuals with newly-diagnosed COPD. Findings from this study indicate that beneficial impact of statin is directly related to duration of statin therapy. Statistically significant reduction COPD-specific emergency room visits and outpatient visits were only observed among beneficiaries who had $\geq 120$ days of statin medication supply during the baseline period.

In addition, our study findings also revealed that statin therapy was beneficial for adults with COPD and inflammation-related multimorbidity (Table 4) as compared to those with inflammation-related multimorbidity and no statin therapy. A plausible explanation could be found in the anti-inflammatory properties of statin therapy. Biologically, statin therapy has shown anti-inflammatory properties by reducing inflammatory biomarkers. (Forrester JS et al, 2007; Gross NJ et al, 2012) As COPD is being recognized as disease of systemic inflammation with high levels of inflammatory biomarkers (P. J. Barnes \& Celli, 2009; Fabbri \& Rabe, 2007; Sevenoaks \& Stockley, 2006), the results from our analyses suggest that the biological benefits of statin medications in reducing systemic inflammation may be spilling over to reduce COPDspecific complications.

Our results indicate that reduction in COPD-specific healthcare outcomes with statin therapy did not translate into statistically significant reduction in total or COPD-specific inpatient or outpatient expenditures. It is plausible that due to inclusion of prescription drug costs in evaluating total expenditures may have offset any cost reduction due to lower healthcare utilization. However, while assessing COPD-specific expenditures, prescriptions costs were not 
included. Future studies with longer follow-up period are required to ascertain whether statin therapy is associated with any economic benefits among those with COPD.

Findings from this study complement the prior evidence that suggests statin use is associated with improved lung function, COPD symptoms and exacerbations. (Blamoun et al., 2008; Huang et al., 2011; Ishida et al., 2007; Keddissi et al., 2007; Lawes et al., 2012) Collectively, these findings warrant the evaluation of statin medications in randomized clinical trials (RCT) setting to establish the efficacy and safety of statin use among individuals with COPD. In fact, few randomized trials that were ongoing when we conceptualized the study have been recently completed. (Criner GJ et al, 2014; NJH, 2014; UHA, 2014) Findings from one of these large, prospective, multicenter randomized trials on evaluating the efficacy of simvastatin on reducing COPD exacerbations differ from the conclusions of our study. (Criner GJ et al, 2014) This may be due to difference in the study population as the randomized controlled trial included individuals with moderate to severe COPD aged between 40-80 years, whereas our study included newly diagnosed COPD patients between 40-64 years of age. Moreover, randomized controlled trial specifically evaluated efficacy of simvastatin. Therefore, more robust evaluation of all statin medications is required in both RCT and real-world practice settings with longer follow-up to confirm the beneficial effects of statins that we observed in our study.

\section{STRENGTHS AND LIMITATIONS}

This study is first real-world observational analyses of clinical and economic outcomes associated with statin therapy among newly-diagnosed COPD patients in a US setting which increases the generalizability of our findings. The major strength of our study includes the use of administrative claims data which provided us the ability to identify all the medical conditions using diagnosis codes. The data also consisted of medication information with NDCs and days of 
medication supply information which helps in determining duration of statin therapy.

Furthermore, our study examines a high-risk and more vulnerable population in terms of Medicaid enrollees. The current study is different from any prior analyses as it evaluated the effects of statin therapy on COPD-specific outcomes.

This study has few limitations such as inability to control for COPD-severity due to unavailability of laboratory data. Our study population was determined using ICD-9-CM codes rather than gold standard approach using spirometry laboratory values. However, the sensitivity and specificity of ICD-9-CM codes to identify COPD has been established in previous studies. The study only used data from only four states which may not provide a full understating of outcomes in overall Medicaid population. We also excluded dual Medicare-Medicaid and managed care enrollees from our population which limits the overall generalizability. Finally, our study is only generalizable to newly-diagnosed COPD patients and thus further research is required to evaluate the effectiveness of statin therapy among individuals with prevalent COPD. 


\section{REFERENCES}

Baigent, C., Keech, A., Kearney, P. M., Blackwell, L., Buck, G., Pollicino, C., Simes, R. (2005). Efficacy and safety of cholesterol-lowering treatment: Prospective meta-analysis of data from 90,056 participants in 14 randomised trials of statins. Lancet, 366(9493), 1267-1278.

Barnes, P. J., \& Celli, B. R. (2009). Systemic manifestations and comorbidities of COPD. The European Respiratory Journal, 33(5), 1165-1185. doi:10.1183/09031936.00128008

Barnes, P. J. (2010). Chronic obstructive pulmonary disease: Effects beyond the lungs. Plos Medicine, 7(3), e1000220-e1000220. doi:10.1371/journal.pmed.1000220

Blamoun, A. I., Batty, G. N., DeBari, V. A., Rashid, A. O., Sheikh, M., \& Khan, M. A. (2008). Statins may reduce episodes of exacerbation and the requirement for intubation in patients with COPD: Evidence from a retrospective cohort study. International Journal of Clinical Practice, 62(9), 1373-1378. doi:10.1111/j.1742-1241.2008.01731.x

Cazzola, M., Bettoncelli, G., Sessa, E., Cricelli, C., \& Biscione, G. (2010). Prevalence of comorbidities in patients with chronic obstructive pulmonary disease. Respiration; International Review of Thoracic Diseases, 80(2), 112-119. doi:10.1159/000281880; 10.1159/000281880

Cazzola, M., Calzetta, L., Bettoncelli, G., Cricelli, C., Romeo, F., Matera, M. G., \& Rogliani, P. (2012). Cardiovascular disease in asthma and COPD: A population-based retrospective cross-sectional study. Respiratory Medicine, 106(2), 249-256. doi:10.1016/j.rmed.2011.07.021; 10.1016/j.rmed.2011.07.021

Cazzola, M., Ciaprini, C., Page, C. P., \& Matera, M. G. (2007). Targeting systemic inflammation: Novel therapies for the treatment of chronic obstructive pulmonary disease. Expert Opinion on Therapeutic Targets, 11(10), 1273-1286.

CMS. (2011). Medicaid analytic eXtract (MAX) general information. (https://www.cms.gov/MedicaidDataSourcesGenInfo/07_MAXGeneralInformation.asp). Baltimore, MD: 50. Centers for Medicare and Medicaid Services, US Department of Health and Human Services.

Cooke, C. R., Joo, M. J., Anderson, S. M., Lee, T. A., Udris, E. M., Johnson, E., \& Au, D. H. (2011). The validity of using ICD-9 codes and pharmacy records to identify patients with chronic obstructive pulmonary disease. BMC Health Services Research, 11, 37-37. doi:10.1186/1472-6963-11-37 
Criner GJ1, Connett JE, Aaron SD, Albert RK, Bailey WC, Casaburi R, Cooper JA Jr, Curtis JL, Dransfield MT, Han MK, Make B, Marchetti N, Martinez FJ, Niewoehner DE, Scanlon PD, Sciurba FC, Scharf SM, Sin DD, Voelker H, Washko GR, Woodruff PG, Lazarus SC; COPD Clinical Research Network; Canadian Institutes of Health Research. Simvastatin for the prevention of exacerbations in moderate-to-severe COPD. N Engl J Med. 2014 Jun 5;370(23):2201-10. Epub 2014 May 18.

Dalal, A. A., Shah, M., D'Souza, A.,O., Chaudhari, S., \& Crater, G. (2012). Clinical and economic outcomes for patients initiating fluticasone propionate/salmeterol combination therapy $(250 / 50 \mathrm{mcg})$ versus anticholinergics in a comorbid COPD/depression population. International Journal of Chronic Obstructive Pulmonary Disease, 7, 11-19. doi:10.2147/COPD.S27846

Fabbri, L. M., \& Rabe, K. F. (2007). From COPD to chronic systemic inflammatory syndrome? Lancet, 370(9589), 797-799.

Forrester JS, Libby P. The inflammation hypothesis and its potential relevance to statin therapy. Am J Cardiol . 2007 ; 99 ( 5 ): 732 - 738 .

Frost, F. J., Petersen, H., Tollestrup, K., \& Skipper, B. (2007). Influenza and COPD mortality protection as pleiotropic, dose-dependent effects of statins. Chest, 131(4), 1006-1012.

Gershon, A. S., Wang, C., Guan, J., Vasilevska-Ristovska, J., Cicutto, L., \& To, T. (2009). Identifying individuals with physician diagnosed COPD in health administrative databases. COPD, 6(5), 388-394.

Global strategy for the diagnosis, management, and prevention on chronic obstructive pulmonary disease: 2013 update. (2013). ().Global Initiative for Chronic Obstructive Pulmonary Disease.

Górska, K., Maskey-Warzechowska, M., \& Krenke, R. (2010). Airway inflammation in chronic obstructive pulmonary disease. Current Opinion in Pulmonary Medicine, 16(2), 89-96. doi:10.1097/MCP.0b013e3283341ba0

Halpern, R., Baker, C. L., Su, J., Woodruff, K. B., Paulose-Ram, R., Porter, V., \& Shah, H. (2011). Outcomes associated with initiation of tiotropium or fluticasone/salmeterol in patients with chronic obstructive pulmonary disease. Patient Preference and Adherence, 5, 375-388. doi:10.2147/PPA.S19991

Healthy people 2020: Goals for respiratory diseases. (2013). Retrieved June 15, 2013, from http://www.healthypeople.gov/2020/topicsobjectives2020/overview.aspx?topicid=36 
Hennessy, S., Leonard, C. E., Palumbo, C. M., Newcomb, C., \& Bilker, W. B. (2007). Quality of medicaid and medicare data obtained through centers for medicare and medicaid services (CMS). Medical Care, 45(12), 1216-1220. doi:10.1097/MLR.0b013e318148435a

Huang, C., Chan, W., Chen, Y., Chen, T., Chou, K., Lin, S., Leu, H. (2011). Statin use and hospitalization in patients with chronic obstructive pulmonary disease: A nationwide population-based cohort study in Taiwan. Clinical Therapeutics, 33(10), 1365-1370. doi:10.1016/j.clinthera.2011.08.010

Ishida, W., Kajiwara, T., Ishii, M., Fujiwara, F., Taneichi, H., Takebe, N., Satoh, J. (2007). Decrease in mortality rate of chronic obstructive pulmonary disease (COPD) with statin use: A population-based analysis in Japan. The Tohoku Journal of Experimental Medicine, 212(3), 265-273.

Keddissi, J. I., Younis, W. G., Chbeir, E. A., Daher, N. N., Dernaika, T. A., \& Kinasewitz, G. T. (2007). The use of statins and lung function in current and former smokers. Chest, 132(6), 1764-1771.

Kozma, C. M., Reeder, C. E., \& Schulz, R. M. (1993). Economic, clinical, and humanistic outcomes: A planning model for pharmacoeconomic research. Clinical Therapeutics, 15(6), 1121-1132.

Lawes, C. M. M., Thornley, S., Young, R., Hopkins, R., Marshall, R., Chan, W. C., \& Jackson, G. (2012). Statin use in COPD patients is associated with a reduction in mortality: A national cohort study. Primary Care Respiratory Journal: Journal of the General Practice Airways Group, 21(1), 35-40. doi:10.4104/pcrj.2011.00095

Lee, J., Lee, D., Kim, E., Choe, K., Oh, Y., Shim, T., . . Lee, S. (2005). Simvastatin inhibits cigarette smoking-induced emphysema and pulmonary hypertension in rat lungs. American Journal of Respiratory and Critical Care Medicine, 172(8), 987-993.

Lee, T., Lin, M., \& Chang, N. (2008). Usefulness of C-reactive protein and interleukin-6 as predictors of outcomes in patients with chronic obstructive pulmonary disease receiving pravastatin. The American Journal of Cardiology, 101(4), 530-535. doi:10.1016/j.amjcard.2007.09.102

Lin, P., Shaya, F. T., \& Scharf, S. M. (2010). Economic implications of comorbid conditions among medicaid beneficiaries with COPD. Respiratory Medicine, 104(5), 697-704. doi:10.1016/j.rmed.2009.11.009 
Make, B., Dutro, M. P., Paulose-Ram, R., Marton, J. P., \& Mapel, D. W. (2012). Undertreatment of COPD: A retrospective analysis of US managed care and medicare patients. International Journal of Chronic Obstructive Pulmonary Disease, 7, 1-9. doi:10.2147/COPD.S27032

Mancini, G. B. J., Etminan, M., Zhang, B., Levesque, L. E., FitzGerald, J. M., \& Brophy, J. M. (2006). Reduction of morbidity and mortality by statins, angiotensin-converting enzyme inhibitors, and angiotensin receptor blockers in patients with chronic obstructive pulmonary disease. Journal of the American College of Cardiology, 47(12), 2554-2560.

Matera, M. G., Calzetta, L., Rinaldi, B., \& Cazzola, M. (2012). Treatment of COPD: Moving beyond the lungs. Current Opinion in Pharmacology, 12(3), 315-322. doi:10.1016/j.coph.2012.04.001

National Jewish Health. Lovastatin as a potential modulator of apoptosis in chronic obstructive pulmonary disease (COPD). NCT00700921. ClinicalTrials.gov. Bethesda, MD: National Institutes of Health; 2008. http://clinicaltrials.gov/ct2/show/NCT00700921

Nussbaumer-Ochsner, Y., \& Rabe, K. F. (2011). Systemic manifestations of COPD. Chest, 139(1), 165-173. doi:10.1378/chest.10-1252

Quality indicator user guide: Prevention quality indicators (PQI) composite measures version 4.3. (2011). ( No. 290-04-0020 (AHRQ SQI-II)). Rockville, MD: U.S. Department of Health and Human Services.

Rabe, K. F., Hurd, S., Anzueto, A., Barnes, P. J., Buist, S. A., Calverley, P., . . Global Initiative for Chronic Obstructive Lung Disease. (2007). Global strategy for the diagnosis, management, and prevention of chronic obstructive pulmonary disease: GOLD executive summary. American Journal of Respiratory and Critical Care Medicine, 176(6), 532-555. doi:10.1164/rccm.200703-456SO

Schnell, K., Weiss, C. O., Lee, T., Krishnan, J. A., Leff, B., Wolff, J. L., \& Boyd, C. (2012). The prevalence of clinically-relevant comorbid conditions in patients with physician-diagnosed COPD: A cross-sectional study using data from NHANES 1999-2008. BMC Pulmonary Medicine, 12, 26-26. doi:10.1186/1471-2466-12-26

Schonbeck, U., \& Libby, P. (2004). Inflammation, immunity, and HMG-CoA reductase inhibitors: Statins as anti-inflammatory agents? Circulation, 109(21 Suppl 1), II18-26. doi:10.1161/01.CIR.0000129505.34151.23

Sevenoaks, M. J., \& Stockley, R. A. (2006). Chronic obstructive pulmonary disease, inflammation and co-morbidity--a common inflammatory phenotype? Respiratory Research, 7, 70-70. 
Sin, D. D., Man, S. F. P., Marciniuk, D. D., Ford, G., FitzGerald, M., Wong, E., . . Rousseau, R. (2008). The effects of fluticasone with or without salmeterol on systemic biomarkers of inflammation in chronic obstructive pulmonary disease. American Journal of Respiratory and Critical Care Medicine, 177(11), 1207-1214. doi:10.1164/rccm.200709-1356OC

Søyseth, V., Brekke, P. H., Smith, P., \& Omland, T. (2007). Statin use is associated with reduced mortality in COPD. The European Respiratory Journal, 29(2), 279-283.

Suda, K., Tsuruta, M., Eom, J., Or, C., Mui, T., Jaw, J., Sin, D. D. (2011). Acute lung injury induces cardiovascular dysfunction: Effects of IL-6 and budesonide/formoterol. American Journal of Respiratory Cell and Molecular Biology, 45(3), 510-516.

doi:10.1165/rcmb.2010-01690C

Takahashi, S., Nakamura, H., Seki, M., Shiraishi, Y., Yamamoto, M., Furuuchi, M., Ishizaka, A. (2008). Reversal of elastase-induced pulmonary emphysema and promotion of alveolar epithelial cell proliferation by simvastatin in mice. American Journal of Physiology.Lung Cellular and Molecular Physiology, 294(5), L882-L890. doi:10.1152/ajplung.00238.2007

University Hospital, Akershus. Effect of rosuvastatin therapy in patients with stable chronic obstructive pulmonary disease (RODEO). NCT00929734. ClinicalTrials.gov. Bethesda, MD: National Institutes of Health; 2009. http://clinicaltrials.gov/ct2/show/NCT00929734

Vogelmeier, C. F., \& Wouters, E. F. M. (2011). Treating the systemic effects of chronic obstructive pulmonary disease. Proceedings of the American Thoracic Society, 8(4), 376379. doi:10.1513/pats.201102-020RM

Walsh, G. M. (2008). Statins as emerging treatments for asthma and chronic obstructive pulmonary disease. Expert Review of Respiratory Medicine, 2(3), 329-335. doi:10.1586/17476348.2.3.329

Young, R. P., Hopkins, R., \& Eaton, T. E. (2009). Pharmacological actions of statins: Potential utility in COPD. European Respiratory Review : An Official Journal of the European Respiratory Society, 18(114), 222-232. doi:10.1183/09059180.00005309; $10.1183 / 09059180.00005309$ 


\section{TABLES AND FIGURES}

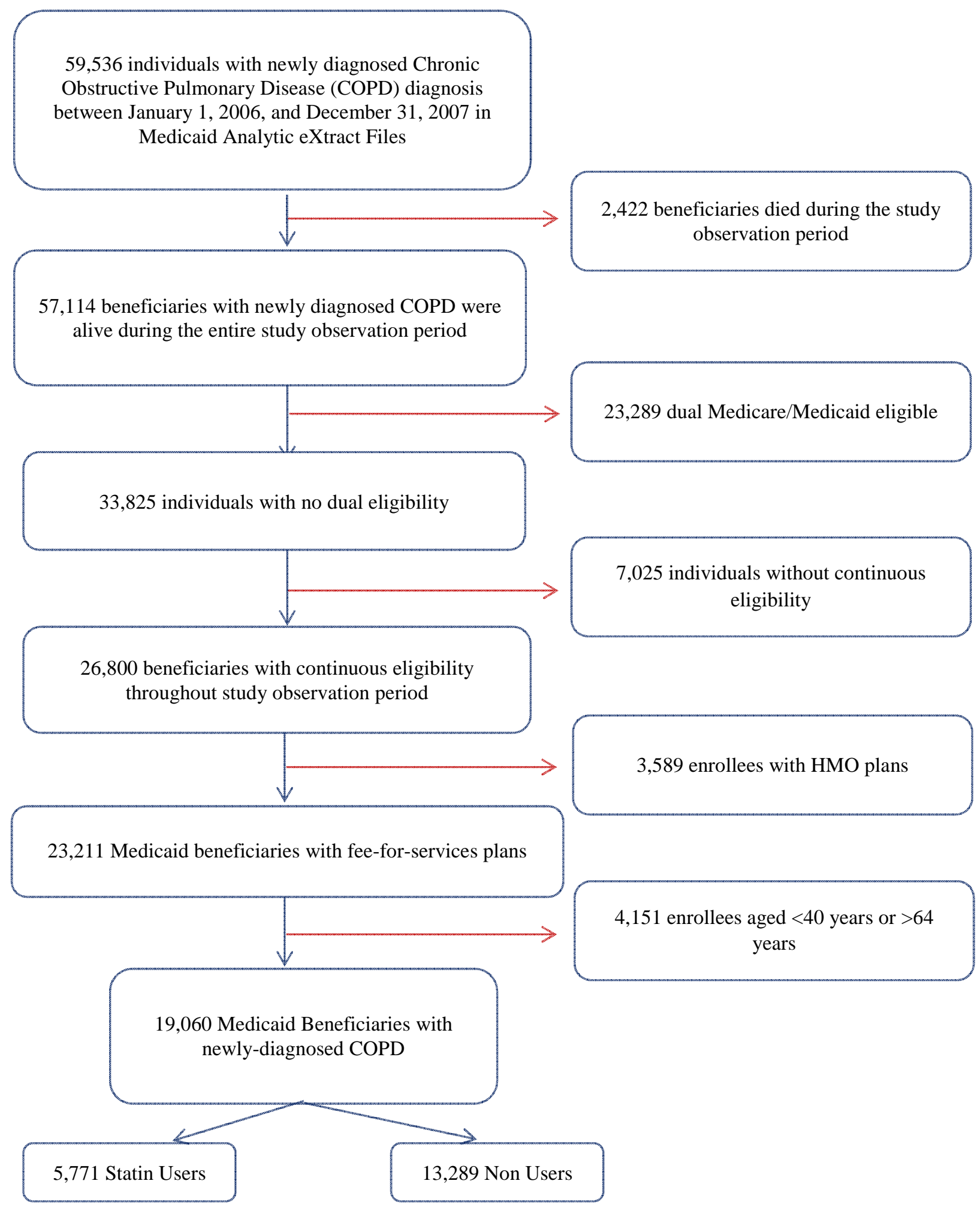

Figure: Algorithm Describing Selection of Study Population 
Table 1 (a)

Number, Percentages, Adjusted Odds Ratio and 95\% CI from Logistic Regressions on Statin Therapy among Medicaid Beneficiaries with Newly-Diagnosed COPD Medicaid Analytic Extract, 2005-2008

\begin{tabular}{|c|c|c|c|c|c|}
\hline & \multicolumn{5}{|c|}{ Statin Therapy } \\
\hline & $\mathbf{N}$ & Row \% & AOR & $95 \% \mathrm{CI}$ & Sig \\
\hline TOTAL & 5,771 & 30.3 & & & \\
\hline \multicolumn{6}{|l|}{ Cohort Year } \\
\hline $2005-2007$ & 3,161 & 29.5 & 0.88 & {$[0.82,0.94]$} & $* * *$ \\
\hline 2006-2008 & 2,610 & 31.3 & & & \\
\hline \multicolumn{6}{|l|}{ Sex } \\
\hline Women & 3,646 & 32.2 & 0.92 & {$[0.86,0.99]$} & $*$ \\
\hline Men & 2,125 & 27.5 & & & \\
\hline \multicolumn{6}{|l|}{ Race/Ethnicity } \\
\hline White & 2,935 & 31.7 & & & \\
\hline African American & 1,231 & 24.2 & 0.61 & {$[0.56,0.67]$} & $* * *$ \\
\hline Others & 1,605 & 34.1 & 0.98 & {$[0.90,1.07]$} & \\
\hline \multicolumn{6}{|l|}{ Age (in years) } \\
\hline $40-49$ & 1,386 & 21.8 & 0.52 & {$[0.47,0.57]$} & $* * *$ \\
\hline $50-59$ & 2,942 & 31.9 & 0.72 & {$[0.66,0.79]$} & $* * *$ \\
\hline $60-64$ & 1,443 & 41.4 & & & \\
\hline \multicolumn{6}{|l|}{ State } \\
\hline California & 3,179 & 30.6 & & & \\
\hline Illinois & 1,257 & 30.6 & 1.07 & {$[0.97,1.19]$} & \\
\hline New York & 854 & 32.7 & 1.22 & {$[1.07,1.38]$} & $* *$ \\
\hline Texas & 481 & 24.8 & 0.87 & {$[0.74,1.02]$} & \\
\hline \multicolumn{6}{|l|}{ Poverty Eligibility } \\
\hline Yes & 5,366 & 30.7 & 1.12 & {$[0.95,1.31]$} & \\
\hline No & 405 & 25.6 & & & \\
\hline \multicolumn{6}{|l|}{ Medical Eligibility } \\
\hline Yes & 512 & 25.1 & 0.91 & {$[0.79,1.06]$} & \\
\hline No & 5,259 & 30.9 & & & \\
\hline \multicolumn{6}{|c|}{ Inflammation-related Multimorbidity } \\
\hline Physical Only & 3,639 & 39.1 & 3.16 & {$[2.85,3.50]$} & $* * *$ \\
\hline Mental Only & 184 & 14.3 & 1.01 & {$[0.84,1.22]$} & \\
\hline Both & 1,345 & 36.0 & 2.75 & {$[2.44,3.10]$} & $* * *$ \\
\hline None & 603 & 12.7 & & & \\
\hline
\end{tabular}


Table 1 (a)

Number, Percentages, Adjusted Odds Ratio and 95\% CI from Logistic Regressions on Statin Therapy among Medicaid Beneficiaries with Newly-Diagnosed COPD Medicaid Analytic Extract, 2005-2008

\begin{tabular}{|c|c|c|c|c|c|}
\hline & \multicolumn{5}{|c|}{ Statin Therapy } \\
\hline & $\mathbf{N}$ & Row \% & AOR & $95 \% \mathrm{CI}$ & Sig \\
\hline TOTAL & 5,771 & 30.3 & & & \\
\hline \multicolumn{6}{|c|}{ Number of Other Clinical Conditions } \\
\hline None & 74 & 15.6 & & & \\
\hline $1-3$ & 835 & 23.5 & 1.23 & {$[0.93,1.63]$} & \\
\hline $4-6$ & 1,268 & 28.3 & 1.15 & {$[0.87,1.52]$} & \\
\hline$>6$ & 3,594 & 34.0 & 0.97 & {$[0.74,1.28]$} & \\
\hline \multicolumn{6}{|c|}{ Serious Mental Illness } \\
\hline Yes & 1,299 & 26.2 & 0.92 & {$[0.85,1.00]$} & \\
\hline No & 4,472 & 31.7 & & & \\
\hline \multicolumn{6}{|c|}{ Alcohol Abuse } \\
\hline Yes & 318 & 17.4 & 0.58 & {$[0.50,0.67]$} & $* * *$ \\
\hline No & 5,453 & 31.6 & & & \\
\hline \multicolumn{6}{|c|}{ Substance Abuse } \\
\hline Yes & 457 & 17.3 & 0.52 & {$[0.46,0.59]$} & $* * *$ \\
\hline No & 5,314 & 32.4 & & & \\
\hline \multicolumn{6}{|c|}{ Tobacco Use } \\
\hline Yes & 675 & 26.6 & 0.98 & {$[0.88,1.10]$} & \\
\hline No & 5,096 & 30.8 & & & \\
\hline \multicolumn{6}{|c|}{ Polypharmacy } \\
\hline Yes & 4,361 & 44.2 & 3.82 & {$[3.52,4.14]$} & $* * *$ \\
\hline No & 1,410 & 15.3 & & & \\
\hline
\end{tabular}

ARF Variables (County Level)

\begin{tabular}{crrcc}
\hline $\begin{array}{c}\text { Above HS Education Density } \\
\text { Q1 }\end{array}$ & 3,168 & 31.5 & & \\
Q2 & 846 & 30.8 & 0.93 & {$[0.81,1.06]$} \\
Q3 & 1,116 & 29.0 & 0.88 & {$[0.77,1.01]$} \\
Q4 & 641 & 26.6 & 0.89 & {$[0.75,1.06]$} \\
Unemployment Density & & & & \\
Q1 & 4,248 & 30.0 & & \\
Q2 & 804 & 31.3 & 1.00 & {$[0.85,1.17]$} \\
Q3 & 515 & 29.0 & 0.79 & {$[0.64,0.97]$} \\
Q4 & 204 & 36.4 & 1.02 & {$[0.75,1.38]$} \\
\end{tabular}


Table 1 (a)

Number, Percentages, Adjusted Odds Ratio and 95\% CI from Logistic Regressions on Statin Therapy among Medicaid Beneficiaries with Newly-Diagnosed COPD Medicaid Analytic Extract, 2005-2008

\begin{tabular}{|c|c|c|c|c|c|}
\hline & \multicolumn{5}{|c|}{ Statin Therapy } \\
\hline & $\mathbf{N}$ & Row \% & AOR & $95 \% \mathrm{CI}$ & Sig \\
\hline TOTAL & $\mathbf{5 , 7 7 1}$ & 30.3 & & & \\
\hline \multicolumn{6}{|c|}{ Poverty Density } \\
\hline Q1 & 423 & 26.7 & & & \\
\hline Q2 & 758 & 28.1 & 1.03 & {$[0.85,1.23]$} & \\
\hline Q3 & 1,075 & 30.6 & 1.13 & {$[0.95,1.34]$} & \\
\hline Q4 & 3,515 & 31.2 & 1.02 & {$[0.84,1.23]$} & \\
\hline \multicolumn{6}{|l|}{ Metro } \\
\hline Yes & 4,984 & 30.2 & 0.89 & {$[0.77,1.04]$} & \\
\hline No & 787 & 31.1 & & & \\
\hline \multicolumn{6}{|c|}{ PCP Shortage } \\
\hline Yes & 5,561 & 30.4 & 1.03 & {$[0.84,1.25]$} & \\
\hline No & 210 & 27.3 & & & \\
\hline \multicolumn{6}{|c|}{ Mental Health Specialist Shortage } \\
\hline Yes & 5,148 & 30.2 & 0.92 & {$[0.80,1.06]$} & \\
\hline No & 623 & 31.1 & & & \\
\hline \multicolumn{6}{|c|}{ PCP Density } \\
\hline Q1 & 261 & 32.5 & & & \\
\hline Q2 & 1,025 & 31.9 & 0.99 & {$[0.79,1.24]$} & \\
\hline Q3 & 805 & 29.8 & 0.88 & {$[0.69,1.13]$} & \\
\hline Q4 & 3,680 & 29.8 & 0.79 & {$[0.58,1.06]$} & \\
\hline \multicolumn{6}{|c|}{ Hospital Beds Density } \\
\hline Q1 & 315 & 31.5 & & & \\
\hline Q2 & 911 & 29.3 & 1.00 & {$[0.82,1.22]$} & \\
\hline Q3 & 3,306 & 30.9 & 1.08 & {$[0.88,1.33]$} & \\
\hline Q4 & 1,239 & 29.2 & 1.03 & {$[0.83,1.27]$} & \\
\hline \multicolumn{6}{|c|}{ Psychiatric Hospital } \\
\hline Yes & 3,699 & 30.6 & 1.05 & {$[0.92,1.19]$} & \\
\hline No & 2,072 & 29.8 & & & \\
\hline \multicolumn{6}{|c|}{ Pulmonologist Density } \\
\hline High & 3,979 & 29.7 & 1.10 & {$[0.94,1.29]$} & \\
\hline Low & 1,792 & 31.6 & & & \\
\hline \multicolumn{6}{|c|}{ Cardiologist Density } \\
\hline High & 3,750 & 30.1 & 1.11 & {$[0.95,1.30]$} & \\
\hline Low & 2,021 & 30.7 & & & \\
\hline
\end{tabular}


Note: Based on 19,060 Medicaid Beneficiaries with newly diagnosed COPD obtained from Medicaid Analytic eXtract files observed during 2005-2008. Asterisks represent significant group differences in likelihood of statin therapy, long-term vs. short-term statin therapy by beneficiary characteristics compared to the reference group (None) obtained from Adjusted Logistic and Adjusted Multinomial Logistic Regression Analyses respectively.

Adjusted Logistic Regression Analyses controlled for cohort year, sex, race, age, state, poverty eligibility, inflammation-related multimorbidity number of other clinical conditions, serious mental illness, alcohol abuse, substance abuse, tobacco use, polypharmacy, county level variables including: above high school education density (quartiles), unemployment density (quartiles), poverty density (quartiles), metro status, primary care shortage area, mental health shortage area, primary care provider density (quartiles), hospital beds density (quartiles), psychiatric hospital, pulmonologist density and cardiologist density.

OR: Odds Ratio; AOR: Adjusted Odds Ratio; CI: Confidence Interval; Sig: Significance

$* * * \mathrm{p}<.001 ; * * .001 \leq \mathrm{p}<.01 ; * .01 \leq \mathrm{p}<.05$ 


\section{Table 1 (b)}

Number, Percentages, Adjusted Odds Ratio and 95\% CI

from Multinomial Logistic Regressions on Duration of Statin Therapy

among Medicaid Beneficiaries with Newly-Diagnosed COPD

Medicaid Analytic Extract, 2005-2008

\begin{tabular}{|c|c|c|c|c|c|c|c|c|c|c|}
\hline & \multicolumn{5}{|c|}{ Long-term Statin therapy } & \multicolumn{5}{|c|}{ Short-term Statin therapy } \\
\hline & $\mathbf{N}$ & $\begin{array}{c}\text { Row } \\
\%\end{array}$ & AOR & $95 \% \mathrm{CI}$ & Sig & $\mathbf{N}$ & $\begin{array}{c}\text { Row } \\
\%\end{array}$ & AOR & $95 \% \mathrm{CI}$ & Sig \\
\hline TOTAL & 4,343 & 22.8 & & & & 1,428 & 7.5 & & & \\
\hline \multicolumn{11}{|l|}{ Cohort Year } \\
\hline $2005-2007$ & 2,377 & 22.2 & 0.87 & {$[0.81,0.94]$} & $* * *$ & 784 & 7.3 & 0.91 & {$[0.81,1.02]$} & \\
\hline $2006-2008$ & 1,966 & 23.6 & & & & 644 & 7.7 & & & \\
\hline \multicolumn{11}{|l|}{ Sex } \\
\hline Women & 2,714 & 23.9 & 0.87 & {$[0.80,0.94]$} & $* * *$ & 932 & 8.2 & 1.08 & {$[0.96,1.22]$} & \\
\hline Men & 1,629 & 21.1 & & & & 496 & 6.4 & & & \\
\hline \multicolumn{11}{|l|}{ Race/Ethnicity } \\
\hline White & 2,320 & 25.0 & & & & 615 & 6.6 & & & \\
\hline African American & 818 & 16.1 & 0.52 & {$[0.47,0.58]$} & $* * *$ & 413 & 8.1 & 0.92 & {$[0.79,1.07]$} & \\
\hline Others & 1,205 & 25.6 & 0.92 & {$[0.84,1.02]$} & & 400 & 8.5 & 1.17 & {$[1.01,1.35]$} & $*$ \\
\hline \multicolumn{11}{|l|}{ Age (in years) } \\
\hline $40-49$ & 973 & 15.3 & 0.45 & {$[0.40,0.50]$} & $* * *$ & 413 & 6.5 & 0.76 & {$[0.64,0.90]$} & $* *$ \\
\hline $50-59$ & 2,199 & 23.8 & 0.67 & {$[0.61,0.73]$} & $* * *$ & 743 & 8.0 & 0.95 & {$[0.82,1.11]$} & \\
\hline $60-64$ & 1,171 & 33.6 & & & & 272 & 7.8 & & & \\
\hline \multicolumn{11}{|l|}{ State } \\
\hline California & 2,384 & 22.9 & & & & 795 & 7.6 & & & \\
\hline Illinois & 917 & 22.3 & 1.10 & {$[0.98,1.23]$} & & 340 & 8.3 & 1.00 & {$[0.84,1.18]$} & \\
\hline New York & 687 & 26.3 & 1.35 & {$[1.17,1.56]$} & $* * *$ & 167 & 6.4 & 0.87 & {$[0.70,1.10]$} & \\
\hline Texas & 355 & 18.3 & 0.86 & {$[0.72,1.03]$} & & 126 & 6.5 & 0.89 & {$[0.68,1.16]$} & \\
\hline \multicolumn{11}{|l|}{ Poverty Eligibility } \\
\hline Yes & 4,061 & 23.2 & 1.21 & {$[1.01,1.46]$} & $*$ & 1,305 & 7.5 & 0.90 & {$[0.70,1.17]$} & \\
\hline No & 282 & 17.8 & & & & 123 & 7.8 & & & \\
\hline \multicolumn{11}{|l|}{ Medical Eligibility } \\
\hline Yes & 367 & 18.0 & 0.92 & {$[0.78,1.09]$} & & 145 & 7.1 & 0.89 & {$[0.70,1.14]$} & \\
\hline No & 3,976 & 23.4 & & & & 1,283 & 7.5 & & & \\
\hline \multicolumn{11}{|c|}{ Inflammation-related Multimorbidity } \\
\hline Physical Only & 2,778 & 29.8 & 3.56 & {$[3.16,4.02]$} & $* * *$ & 861 & 9.3 & 2.35 & {$[1.98,2.78]$} & $* * *$ \\
\hline Mental Only & 124 & 9.7 & 1.00 & {$[0.80,1.25]$} & & 60 & 4.7 & 1.04 & {$[0.77,1.41]$} & \\
\hline Both & 1,033 & 27.7 & 3.12 & {$[2.72,3.59]$} & $* * *$ & 312 & 8.4 & 2.01 & {$[1.64,2.45]$} & $* * *$ \\
\hline None & 408 & 8.6 & & & & 195 & 4.1 & & & \\
\hline
\end{tabular}

(Continued) 


\section{Table 1 (b)}

Number, Percentages, Adjusted Odds Ratio and 95\% CI

from Multinomial Logistic Regressions on Duration of Statin Therapy

among Medicaid Beneficiaries with Newly-Diagnosed COPD

Medicaid Analytic Extract, 2005-2008

\begin{tabular}{|c|c|c|c|c|c|c|c|c|c|c|}
\hline & \multicolumn{5}{|c|}{ Long-term Statin therapy } & \multicolumn{5}{|c|}{ Short-term Statin therapy } \\
\hline & $\mathbf{N}$ & $\begin{array}{c}\text { Row } \\
\%\end{array}$ & AOR & $95 \% \mathrm{CI}$ & Sig & $\mathbf{N}$ & $\begin{array}{c}\text { Row } \\
\%\end{array}$ & AOR & $95 \% \mathrm{CI}$ & Sig \\
\hline TOTAL & 4,343 & 22.8 & & & & 1,428 & 7.5 & & & \\
\hline \multicolumn{11}{|c|}{ Number of Other Clinical Conditions } \\
\hline None & 51 & 10.8 & & & & 23 & 4.9 & & & \\
\hline $1-3$ & 623 & 17.6 & 1.32 & {$[0.96,1.83]$} & & 212 & 6.0 & 1.03 & {$[0.66,1.62]$} & \\
\hline $4-6$ & 947 & 21.2 & 1.22 & {$[0.88,1.69]$} & & 321 & 7.2 & 1.00 & {$[0.64,1.56]$} & \\
\hline$>6$ & 2,722 & 25.8 & 1.03 & {$[0.75,1.42]$} & & 872 & 8.3 & 0.85 & {$[0.54,1.32]$} & \\
\hline \multicolumn{11}{|c|}{ Serious Mental Illness } \\
\hline Yes & 998 & 20.1 & 0.98 & {$[0.90,1.08]$} & & 301 & 6.1 & 0.77 & {$[0.67,0.89]$} & $* * *$ \\
\hline No & 3,345 & 23.7 & & & & 1,127 & 8.0 & & & \\
\hline \multicolumn{11}{|c|}{ Alcohol Abuse } \\
\hline Yes & 209 & 11.5 & 0.51 & {$[0.44,0.61]$} & $* * *$ & 109 & 6.0 & 0.76 & {$[0.61,0.95]$} & $*$ \\
\hline No & 4,134 & 24.0 & & & & 1,319 & 7.7 & & & \\
\hline \multicolumn{11}{|c|}{ Substance Abuse } \\
\hline Yes & 293 & 11.1 & 0.45 & {$[0.39,0.52]$} & $* * *$ & 164 & 6.2 & 0.70 & {$[0.58,0.84]$} & $* * *$ \\
\hline No & 4,050 & 24.7 & & & & 1,264 & 7.7 & & & \\
\hline \multicolumn{11}{|c|}{ Tobacco Use } \\
\hline Yes & 470 & 18.5 & 0.90 & {$[0.80,1.02]$} & & 205 & 8.1 & 1.21 & {$[1.03,1.43]$} & $*$ \\
\hline No & 3,873 & 23.4 & & & & 1,223 & 7.4 & & & \\
\hline \multicolumn{11}{|c|}{ Polypharmacy } \\
\hline Yes & 3,355 & 34.0 & 4.19 & {$[3.82,4.59]$} & $* * *$ & 1,006 & 10.2 & 3.00 & {$[2.62,3.44]$} & $* * *$ \\
\hline No & 988 & 10.7 & & & & 422 & 4.6 & & & \\
\hline
\end{tabular}

ARF Variables (County Level)

\begin{tabular}{|c|c|c|c|c|c|c|c|c|c|c|}
\hline \multicolumn{11}{|c|}{ Above HS Education Density } \\
\hline Q1 & 2,327 & 23.1 & & & & 841 & 8.4 & & & \\
\hline Q2 & 638 & 23.2 & 0.95 & {$[0.81,1.10]$} & & 208 & 7.6 & 0.88 & {$[0.70,1.10]$} & \\
\hline Q3 & 876 & 22.8 & 0.95 & {$[0.82,1.10]$} & & 240 & 6.2 & 0.70 & {$[0.55,0.89]$} & $* *$ \\
\hline Q4 & 502 & 20.8 & 0.95 & {$[0.78,1.14]$} & & 139 & 5.8 & 0.75 & {$[0.56,1.01]$} & \\
\hline \multicolumn{11}{|c|}{ Unemployment Density } \\
\hline Q1 & 3,166 & 22.4 & & & & 1,082 & 7.6 & & & \\
\hline Q2 & 607 & 23.6 & 0.98 & {$[0.82,1.16]$} & & 197 & 7.7 & 1.07 & {$[0.81,1.42]$} & \\
\hline Q3 & 406 & 22.9 & 0.79 & {$[0.63,0.99]$} & $*$ & 109 & 6.1 & 0.77 & {$[0.54,1.10]$} & \\
\hline Q4 & 164 & 29.3 & 1.02 & {$[0.73,1.42]$} & & 40 & 7.1 & 1.00 & {$[0.59,1.71]$} & \\
\hline
\end{tabular}

(Continued) 


\section{Table 1 (b)}

Number, Percentages, Adjusted Odds Ratio and 95\% CI

from Multinomial Logistic Regressions on Duration of Statin Therapy

among Medicaid Beneficiaries with Newly-Diagnosed COPD

Medicaid Analytic Extract, 2005-2008

\begin{tabular}{|c|c|c|c|c|c|c|c|c|c|c|}
\hline & \multicolumn{5}{|c|}{ Long-term Statin therapy } & \multicolumn{5}{|c|}{ Short-term Statin therapy } \\
\hline & $\mathbf{N}$ & $\begin{array}{c}\text { Row } \\
\%\end{array}$ & AOR & $95 \% \mathrm{CI}$ & Sig & $\mathbf{N}$ & $\begin{array}{c}\text { Row } \\
\%\end{array}$ & AOR & $95 \% \mathrm{CI}$ & Sig \\
\hline TOTAL & 4,343 & 22.8 & & & & 1,428 & 7.5 & & & \\
\hline \multicolumn{11}{|c|}{ Poverty Density } \\
\hline Q1 & 339 & 21.4 & & & & 84 & 5.3 & & & \\
\hline Q2 & 577 & 21.4 & 0.97 & {$[0.79,1.18]$} & & 181 & 6.7 & 1.25 & {$[0.90,1.74]$} & \\
\hline Q3 & 827 & 23.6 & 1.09 & {$[0.90,1.32]$} & & 248 & 7.1 & 1.25 & {$[0.91,1.72]$} & \\
\hline Q4 & 2,600 & 23.1 & 1.00 & {$[0.81,1.23]$} & & 915 & 8.1 & 1.09 & {$[0.77,1.55]$} & \\
\hline \multicolumn{11}{|l|}{ Metro } \\
\hline Yes & 3,720 & 22.5 & 0.86 & {$[0.73,1.02]$} & & 1,264 & 7.6 & 1.00 & {$[0.77,1.31]$} & \\
\hline No & 623 & 24.6 & & & & 164 & 6.5 & & & \\
\hline \multicolumn{11}{|c|}{ PCP Shortage } \\
\hline Yes & 4,188 & 22.9 & 1.16 & {$[0.93,1.44]$} & & 1,373 & 7.5 & 0.73 & {$[0.53,1.01]$} & \\
\hline No & 155 & 20.1 & & & & 55 & 7.1 & & & \\
\hline \multicolumn{11}{|c|}{ Mental Health Specialist Shortage } \\
\hline Yes & 3,854 & 22.6 & 0.94 & {$[0.80,1.09]$} & & 1,294 & 7.6 & 0.88 & {$[0.69,1.12]$} & \\
\hline No & 489 & 24.4 & & & & 134 & 6.7 & & & \\
\hline \multicolumn{11}{|c|}{ PCP Density } \\
\hline Q1 & 205 & 25.5 & & & & 56 & 7.0 & & & \\
\hline Q2 & 770 & 24.0 & 0.96 & {$[0.75,1.22]$} & & 255 & 7.9 & 1.11 & {$[0.75,1.65]$} & \\
\hline Q3 & 629 & 23.3 & 0.89 & {$[0.68,1.17]$} & & 176 & 6.5 & 0.86 & {$[0.56,1.32]$} & \\
\hline Q4 & 2,739 & 22.2 & 0.81 & {$[0.58,1.12]$} & & 941 & 7.6 & 0.75 & {$[0.45,1.28]$} & \\
\hline \multicolumn{11}{|c|}{ Hospital Beds Density } \\
\hline Q1 & 242 & 24.2 & & & & 73 & 7.3 & & & \\
\hline Q2 & 704 & 22.6 & 1.04 & {$[0.83,1.29]$} & & 207 & 6.7 & 0.89 & {$[0.63,1.25]$} & \\
\hline Q3 & 2,431 & 22.7 & 1.08 & {$[0.86,1.36]$} & & 875 & 8.2 & 1.05 & {$[0.74,1.49]$} & \\
\hline Q4 & 966 & 22.8 & 1.05 & {$[0.83,1.32]$} & & 273 & 6.4 & 0.95 & {$[0.66,1.36]$} & \\
\hline \multicolumn{11}{|c|}{ Psychiatric Hospital } \\
\hline Yes & 2,739 & 22.6 & 1.03 & {$[0.89,1.18]$} & & 960 & 7.9 & 1.12 & {$[0.91,1.38]$} & \\
\hline No & 1,604 & 23.1 & & & & 468 & 6.7 & & & \\
\hline \multicolumn{11}{|c|}{ Pulmonologist Density } \\
\hline High & 2,959 & 22.1 & 1.06 & {$[0.89,1.26]$} & & 1,020 & 7.6 & 1.23 & {$[0.93,1.62]$} & \\
\hline Low & 1,384 & 24.4 & & & & 408 & 7.2 & & & \\
\hline \multicolumn{11}{|c|}{ Cardiologist Density } \\
\hline High & 2,797 & 22.4 & 1.12 & {$[0.94,1.33]$} & & 953 & 7.6 & 1.08 & {$[0.82,1.42]$} & \\
\hline Low & 1,546 & 23.5 & & & & 475 & 7.2 & & & \\
\hline
\end{tabular}


Note: Based on 19,060 Medicaid Beneficiaries with newly diagnosed COPD obtained from Medicaid Analytic eXtract files observed during 2005-2008. Asterisks represent significant group differences in likelihood of statin therapy, long-term vs. short-term statin therapy by beneficiary characteristics compared to the reference group (None) obtained from Adjusted Multinomial Logistic Regression Analyses respectively.

Adjusted Multinomial Logistic Regression Analyses controlled for cohort year, sex, race, age, state, poverty eligibility, inflammation-related multimorbidity number of other clinical conditions, serious mental illness, alcohol abuse, substance abuse, tobacco use, polypharmacy, county level variables including: above high school education density (quartiles), unemployment density (quartiles), poverty density (quartiles), metro status, primary care shortage area, mental health shortage area, primary care provider density (quartiles), hospital beds density (quartiles), psychiatric hospital, pulmonologist density and cardiologist density.

OR: Odds Ratio; AOR: Adjusted Odds Ratio; CI: Confidence Interval; Sig: Significance

$* * * \mathrm{p}<.001 ; * * .001 \leq \mathrm{p}<.01 ; * .01 \leq \mathrm{p}<.05$ 
Table 2

Number, Percentage, Adjusted Odds Ratio and 95\% Confidence Interval from Logistic

Regressions on COPD-specific Healthcare Utilization among

Medicaid Beneficiaries with Newly-Diagnosed COPD $(n=19,060)$

Medicaid Analytic Extract, 2005-2008

\begin{tabular}{|c|c|c|c|c|c|c|}
\hline \multicolumn{7}{|c|}{ COPD-specific Hospitalizations } \\
\hline & $\mathbf{N}$ & Row \% & Sig & AOR & $95 \% \mathrm{CI}$ & Sig \\
\hline TOTAL & 998 & 5.2 & & & & \\
\hline Statin therapy & & & $*$ & & & \\
\hline Yes & 270 & 4.7 & & 0.84 & {$[0.71,0.98]$} & * \\
\hline No & 728 & 5.5 & & & & \\
\hline Duration of Statin therapy & & & & & & \\
\hline Long-term & 204 & 4.7 & $*$ & 0.84 & {$[0.71,0.99]$} & $*$ \\
\hline Short-term & 66 & 4.6 & & 0.81 & {$[0.62,1.05]$} & \\
\hline No Use & 728 & 5.5 & & & & \\
\hline \multicolumn{7}{|c|}{ COPD-specific ER visits } \\
\hline TOTAL & 2,824 & 14.8 & & & & \\
\hline Statin therapy & & & $* * *$ & & & \\
\hline Yes & 775 & 13.4 & & 0.89 & {$[0.81,0.99]$} & $*$ \\
\hline No & 2,049 & 15.4 & & & & \\
\hline Duration of Statin therapy & & & $* * *$ & & & \\
\hline Long-term & 564 & 13.0 & & 0.86 & {$[0.77,0.96]$} & $* *$ \\
\hline Short-term & 211 & 14.8 & & 0.99 & {$[0.85,1.16]$} & \\
\hline No Use & 2,049 & 15.4 & & & & \\
\hline \multicolumn{7}{|c|}{ COPD-specific Outpatient Visits } \\
\hline TOTAL & 7,200 & 43.7 & & & & \\
\hline Statin therapy & & & $* * *$ & & & \\
\hline Yes & 2,032 & 41.4 & & 0.86 & {$[0.80,0.92]$} & $* * *$ \\
\hline No & 5,168 & 44.7 & & & & \\
\hline Duration of Statin therapy & & & $* * *$ & & & \\
\hline Long-term & 1,515 & 41.2 & & 0.84 & {$[0.78,0.92]$} & $* * *$ \\
\hline Short-term & 517 & 41.8 & & 0.90 & {$[0.80,1.02]$} & \\
\hline No Use & 5,168 & 44.7 & & & & \\
\hline
\end{tabular}

Note: Based on 19,060 Medicaid Beneficiaries with newly diagnosed COPD obtained from Medicaid Analytic eXtract files observed during 2005-2008. Asterisks represent significant group differences in likelihood of COPD-specific healthcare utilization by statin therapy and duration of statin therapy compared to the reference group (None) obtained from Adjusted Logistic Regression analyses.

Adjusted Logistic Regression Analyses controlled for cohort year, sex, race, age, state, poverty eligibility, inflammation-related multimorbidity number of other clinical conditions, serious mental illness, alcohol abuse, substance abuse, tobacco use, polypharmacy, county level variables including: above high school education density (quartiles), unemployment density (quartiles), poverty density (quartiles), metro status, primary care shortage area, 
mental health shortage area, primary care provider density (quartiles), hospital beds density (quartiles), psychiatric hospital, pulmonologist density and cardiologist density.

OR: Odds Ratio; AOR: Adjusted Odds Ratio; CI: Confidence Interval; Sig: Significance $* * * \mathrm{p}<.001 ; * * .001 \leq \mathrm{p}<.01 ; * .01 \leq \mathrm{p}<.05$ 
Table 3

Adjusted Odds Ratio and 95\% Confidence Interval from Logistic Regressions on COPDspecific Healthcare Utilization

among Medicaid Beneficiaries with Newly-Diagnosed COPD (n = 19, 060)

Inverse Probability Treatment Weight Adjustment

Medicaid Analytic Extract, 2005-2008

COPD-specific Hospitalizations

\begin{tabular}{|c|c|c|c|}
\hline & AOR & $95 \% \mathrm{CI}$ & Sig \\
\hline \multicolumn{4}{|l|}{ Statin therapy } \\
\hline Yes & 0.76 & {$[0.66,0.87]$} & $* * *$ \\
\hline \multicolumn{4}{|l|}{ No } \\
\hline \multicolumn{4}{|c|}{ Duration of Statin therapy } \\
\hline Long-term & 0.73 & {$[0.66,0.80]$} & $* * *$ \\
\hline Short-term & 0.83 & {$[0.75,0.91]$} & $* * *$ \\
\hline \multicolumn{4}{|l|}{ No Use } \\
\hline \multicolumn{4}{|c|}{ COPD-specific ER visits } \\
\hline \multicolumn{4}{|l|}{ Statin therapy } \\
\hline Yes & 0.81 & {$[0.75,0.89]$} & $* * *$ \\
\hline \multicolumn{4}{|l|}{ No } \\
\hline \multicolumn{4}{|c|}{ Duration of Statin therapy } \\
\hline Long-term & 0.75 & {$[0.67,0.83]$} & $* * *$ \\
\hline Short-term & 0.95 & {$[0.81,1.11]$} & \\
\hline No Use & & & \\
\hline
\end{tabular}

COPD-specific Outpatient Visits

\section{Statin therapy}

Yes $\quad 0.86 \quad[0.80,0.91] \quad * * *$

No

Duration of Statin therapy

$\begin{array}{llc}\text { Long-term } & 0.84 & {[0.77,0.90]} \\ \text { Short-term } & 0.89 & {[0.79,1.00]} \\ \text { No Use } & & \end{array}$

Note: Based on 19,060 Medicaid Beneficiaries with newly diagnosed COPD obtained from Medicaid Analytic eXtract files observed during 2005-2008. Asterisks represent significant group differences in likelihood of COPD-specific healthcare utilization by statin therapy and duration of statin therapy compared to the reference group (None).

Adjusted Logistic Analyses controlled for cohort year, sex, race, age, state, poverty eligibility, inflammation-related multimorbidity number of other clinical conditions, serious mental illness, alcohol, substance abuse, tobacco use, polypharmacy, county variables including: above high school education (quartiles), unemployment density (quartiles), poverty density (quartiles), metro status, primary care shortage area, mental health shortage area, primary care provider density (quartiles), hospital beds density (quartiles), psychiatric hospital, pulmonologist density and cardiologist density.

OR: Odds Ratio; AOR: Adjusted Odds Ratio; CI: Confidence Interval; Sig: Significance

$* * * \mathrm{p}<.001 ; * * .001 \leq \mathrm{p}<.01 ; * .01 \leq \mathrm{p}<.05$ 
Table 4

Adjusted Odds Ratio and 95\% Confidence Interval from Logistic Regressions on COPD-specific Healthcare Utilization among Medicaid Beneficiaries with Newly-Diagnosed COPD $(n=19,060)$ Medicaid Analytic Extract, 2005-2008

\begin{tabular}{|c|c|c|c|}
\hline \multicolumn{4}{|c|}{ COPD-specific Hospitalizations } \\
\hline & AOR & $95 \% \mathrm{CI}$ & Sig \\
\hline \multicolumn{4}{|c|}{ Inflammation-related Multimorbidity/Statin therapy } \\
\hline MM and Statin therapy & 0.82 & {$[0.70,0.97]$} & $*$ \\
\hline \multicolumn{4}{|l|}{ MM and no Statin therapy } \\
\hline No MM and Statin therapy & 0.98 & {$[0.67,1.46]$} & \\
\hline No MM and no Statin therapy & 1.17 & {$[0.98,1.39]$} & \\
\hline \multicolumn{4}{|c|}{ COPD-specific Emergency Room visits } \\
\hline \multicolumn{4}{|c|}{ Inflammation-related Multimorbidity/Statin therapy } \\
\hline Statin and MM & 0.90 & {$[0.81,0.99]$} & $*$ \\
\hline \multicolumn{4}{|l|}{ No Statin and MM } \\
\hline Statin and no MM & 0.99 & {$[0.77,1.27]$} & \\
\hline No statin and no MM & 1.17 & {$[1.05,1.31]$} & $* *$ \\
\hline \multicolumn{4}{|c|}{ COPD-specific Outpatient Visits } \\
\hline \multicolumn{4}{|c|}{ Inflammation-related Multimorbidity/Statin therapy } \\
\hline Statin and MM & 0.85 & {$[0.78,0.92]$} & $* * *$ \\
\hline \multicolumn{4}{|l|}{ No Statin and MM } \\
\hline Statin and no MM & 0.88 & {$[0.73,1.05]$} & \\
\hline No statin and no MM & 0.95 & {$[0.87,1.03]$} & \\
\hline
\end{tabular}

Note: Based on 19,060 Medicaid Beneficiaries with newly diagnosed COPD obtained from Medicaid Analytic eXtract files observed during 2005-2008. Asterisks represent significant group differences in likelihood of COPD-specific healthcare utilization by statin therapy/multimorbidity interaction categories compared to the reference group (No statin and no multimorbidity) obtained from Adjusted Logistic Regression analyses.

Adjusted Logistic regressions controlled for cohort year, sex, race, age, state, poverty eligibility, inflammation-related multimorbidity number of other clinical conditions, serious mental illness, alcohol abuse, substance abuse, tobacco use, polypharmacy, county level variables including: above high school education density (quartiles), unemployment density (quartiles), poverty density (quartiles), metro status, primary care shortage area, mental health shortage area, primary care provider density (quartiles), hospital beds density (quartiles), psychiatric hospital, pulmonologist density and cardiologist density.

MM: Inflammation-related multimorbidity; OR: Odds Ratio; AOR: Adjusted Odds Ratio; CI: Confidence Interval; Sig: Significance

$* * * \mathrm{p}<.001 ; * * .001 \leq \mathrm{p}<.01 ; * .01 \leq \mathrm{p}<.05$ 


\begin{tabular}{|c|c|c|c|c|c|c|}
\hline \multicolumn{7}{|c|}{$\begin{array}{c}\text { Table } 5 \\
\text { Mean, Standard Deviation, Ratio of Means of Expenditures Between Statins } \\
\text { Users and Non-Users (Selected Characteristics) } \\
\text { among Medicaid Beneficiaries with Newly-Diagnosed COPD } \\
\text { Medicaid Analytic Extract, 2005-2008 }\end{array}$} \\
\hline \multicolumn{7}{|c|}{ Total Healthcare Expenditures } \\
\hline & \multicolumn{2}{|c|}{ Statin therapy } & \multicolumn{2}{|c|}{ No Statin therapy } & \multirow[b]{2}{*}{ ROM } & \multirow[b]{2}{*}{ Sig } \\
\hline & Mean & SD & Mean & SD & & \\
\hline ALL & 36,215 & 38,329 & 35,724 & 43,549 & 1.01 & \\
\hline \multicolumn{7}{|c|}{ Inflammation-related Multimorbidity } \\
\hline Physical Only & 35,867 & 40,956 & 36,956 & 45,196 & 0.97 & \\
\hline Mental Only & 35,450 & 25,875 & 33,129 & 31,999 & 1.07 & \\
\hline Both & 39,610 & 35,451 & 41,955 & 51,163 & 0.94 & \\
\hline None & 28,120 & 24,660 & 28,224 & 33,538 & 1.00 & \\
\hline \multicolumn{7}{|c|}{ COPD-specific Inpatient Expenditures } \\
\hline ALL & 6,593 & 5,814 & 7,457 & 13,032 & 0.88 & $*$ \\
\hline \multicolumn{7}{|c|}{ Inflammation-related Multimorbidity } \\
\hline Physical Only & 6,446 & 5,573 & 7,594 & 16,204 & 0.85 & * \\
\hline Mental Only & 4,434 & 2,537 & 6,151 & 7,973 & 0.72 & \\
\hline Both & 6,958 & 6,417 & 6,143 & 5,969 & 1.13 & \\
\hline None & 7,556 & 6,761 & 8,535 & 12,590 & 0.89 & \\
\hline \multicolumn{7}{|c|}{ COPD-specific Outpatient Expenditures } \\
\hline ALL & 429 & 1,380 & 423 & 1,631 & 1.01 & \\
\hline \multicolumn{7}{|c|}{ Inflammation-related Multimorbidity } \\
\hline Physical Only & 451 & 1,554 & 409 & 1,252 & 1.10 & $* *$ \\
\hline Mental Only & 349 & 865 & 376 & 1,429 & 0.93 & \\
\hline Both & 405 & 1,082 & 431 & 1,878 & 0.94 & \\
\hline None & 377 & 968 & 450 & 1,948 & 0.84 & $* *$ \\
\hline
\end{tabular}

Note: Based 19,060 Medicaid Beneficiaries with newly diagnosed COPD obtained from Medicaid Analytic eXtract files observed during 2005-2008. Asterisks represent significant differences in average total and average COPDspecific healthcare expenditures by statin therapy obtained from unadjusted GLM among those with positive expenditures.

SD: Standard Deviation; ROM: Ratio of Means Sig: Significance

$* * * \mathrm{p}<.001 ; * * .001 \leq \mathrm{p}<.01 ; * .01 \leq \mathrm{p}<.05$ 
Table 6

Description of Total and COPD-specific healthcare expenditures among Medicaid

Beneficiaries with Newly Diagnosed COPD

among Medicaid Beneficiaries with Newly-Diagnosed COPD

Medicaid Analytic Extract, 2005-2008

\begin{tabular}{|c|c|c|c|c|}
\hline \multicolumn{5}{|c|}{ Total Healthcare Expenditure } \\
\hline & Adjusted $\beta$ & SE & $\operatorname{Exp}(\beta)$ & Sig \\
\hline Intercept & 9.08 & 0.11 & 8,739 & $* * *$ \\
\hline \multicolumn{5}{|l|}{ Statin therapy } \\
\hline Yes & 0.03 & 0.02 & 8,979 & \\
\hline \multicolumn{5}{|l|}{ No } \\
\hline Intercept & 9.08 & 0.11 & 8,759 & $* * *$ \\
\hline \multicolumn{5}{|c|}{ Duration of Statin therapy } \\
\hline Long-term & 0.07 & 0.02 & 9,394 & $* * *$ \\
\hline Short-term & -0.10 & 0.03 & 7,902 & $* * *$ \\
\hline \multicolumn{5}{|l|}{ No use } \\
\hline \multicolumn{5}{|c|}{ COPD-specific Inpatient Expenditures (n = 998) } \\
\hline Intercept & 8.00 & 0.39 & 2,966 & $* * *$ \\
\hline \multicolumn{5}{|l|}{ Statin therapy } \\
\hline Yes & -0.08 & 0.06 & 2,751 & \\
\hline \multicolumn{5}{|l|}{ No } \\
\hline Intercept & 7.98 & 0.40 & 2,929 & $* * *$ \\
\hline \multicolumn{5}{|c|}{ Duration of Statin therapy } \\
\hline Long-term & -0.09 & 0.07 & 2,684 & \\
\hline Short-term & -0.04 & 0.10 & 2,809 & \\
\hline \multicolumn{5}{|l|}{ No use } \\
\hline \multicolumn{5}{|c|}{ COPD-specific Outpatient Expenditures } \\
\hline Intercept & 6.76 & 0.15 & 865 & $* * *$ \\
\hline \multicolumn{5}{|l|}{ Statin therapy } \\
\hline Yes & -0.01 & 0.02 & 857 & \\
\hline \multicolumn{5}{|l|}{ No } \\
\hline Intercept & 6.76 & 0.15 & 866 & $* * *$ \\
\hline \multicolumn{5}{|c|}{ Duration of Statin therapy } \\
\hline Long-term & 0.02 & 0.03 & 882 & \\
\hline Short-term & -0.09 & 0.04 & 792 & * \\
\hline No use & & & & \\
\hline
\end{tabular}

Note: Based on 19,060 Medicaid Beneficiaries with newly diagnosed COPD obtained from Medicaid Analytic eXtract files observed during 2005-2008. Asterisks represent significant group differences in expenditures by statin therapy and duration of statin therapy compared to the reference group (None) obtained from Unadjusted and Adjusted Generalized Linear Model with Log-Link and Gamma Distribution. 
GLM analyses controlled for cohort year, sex, race, age, state, poverty eligibility, inflammation-related multimorbidity number of other clinical conditions, serious mental illness, alcohol abuse, substance abuse, tobacco use, polypharmacy, county level variables including: above high school education density (quartiles), unemployment density

(quartiles), poverty density (quartiles), metro status, primary care shortage area, mental health shortage area, primary care provider density (quartiles), hospital beds density (quartiles), psychiatric hospital, pulmonologist density and cardiologist density.

Exp: Exponentiation SE: Standard Error; Sig: Significance; $\beta$ : Parameter Estimates

$* * * \mathrm{p}<.001 ; * * .001 \leq \mathrm{p}<.01 ; * .01 \leq \mathrm{p}<.05$ 
CHAPTER 4 


\section{CHAPTER 4: ANTIDEPRESSANTS, INHALED CORTICOSTEROIDS, STATINS AND NEW-ONSET DIABETES AMONG MEDICAID BENEFICIARIES WITH NEWLY- DIAGNOSED COPD}

\section{INTRODUCTION}

Chronic Obstructive Pulmonary Disease (COPD) is an inflammatory disease linked to persistent and progressive airflow limitation. (Górska, Maskey-Warzechowska, \& Krenke, 2010; Rabe et al., 2007) COPD is increasingly being recognized as "chronic systemic inflammatory syndrome" (Fabbri \& Rabe, 2007). Individuals with COPD have high levels of inflammatory biomarkers that may be either due to the spill-over effect from the pulmonary inflammation or due to overall "systemic" inflammation caused by presence of inflammation-related chronic conditions such as arthritis, cardiovascular diseases (CVD), depression, diabetes and osteoporosis. (P. J. Barnes \& Celli, 2009; Fabbri \& Rabe, 2007)

COPD is primarily managed through pharmacological treatment. Therefore it is not surprising that use of respiratory disease medications has been growing. The global markets for respiratory disease medications has been increasing and is expected to grow at a compound annual growth rate of $4.4 \%$ to reach $\$ 47.1$ billion by 2017. (Lehr, 2012) Similarly, the use of medications to treat inflammation-related multimorbidity among individuals for COPD has been increasing. Statins used among individuals with cardiovascular diseases and antidepressants used among those with depression are the most prescribed classes of medication in the United States (US). (Mann, Reynolds, Smith, \& Muntner, 2008; Mojtabai \& Olfson, 2011; Olfson \& Marcus, 2009; Paulose-Ram, Safran, Jonas, Gu, \& Orwig, 2007) Both these medications are extensively used among younger adults as well. (Mann et al., 2008; Olfson \& Marcus, 2009) There are many reasons for multiple medication use among those with COPD. In general, medication use among COPD has been increasing (Lehr, 2012) individuals with COPD are more likely to have 
multimorbidity compared to those without COPD and the likelihood of multiple medication use increases with presence of multimorbidity. (Schnell et al., 2012) Using a cross-sectional study among individuals with COPD $(n=126,283)$, researchers have reported that $98 \%$ of individuals received at least one prescription of "non-respiratory drugs". In the same study, $64 \%$ cardiovascular medication and $8 \%$ depression medication use was reported. (Anecchino et al., 2007)

Although, generally regarded as safe, concerns about adverse effects have been raised about some of these medications in recent years. Specifically, antidepressants, inhaled corticosteroids (ICS) and statins have been linked with new-onset diabetes. (Bhattacharjee, Bhattacharya, Kelley, \& Sambamoorthi, 2013; O'Byrne et al., 2012; Sattar et al., 2010; Suissa, Kezouh, \& Ernst, 2010) New-onset diabetes has been linked with medication use because some of these medications can increase insulin resistance and decrease insulin secretion and thereby affecting the overall glucose metabolism. (Carvalho et al., 2004; Colbert \& Stone, 2012; Nielsen et al., 2004)

\section{General Biological Correlates of Medications and New-Onset Diabetes}

Biological relationship between statin use and risk of developing diabetes has been poorly understood. Some investigators have suggested that lipophillic statins like atorvastatin and simvastatin may reduce insulin secretions and potentially affect glucose metabolism. (Colbert \& Stone, 2012) With respect to insulin sensitivity or insulin resistance, the statin effects are different based on type of statins. (Kanda, Satoh, \& Ichihara, 2003; Nakata et al., 2006), simvastatin and lovastatin reduces insulin sensitivity by affect cholesterol synthetic pathway; (Chamberlain, 2001; Koh, Sakuma, \& Quon, 2011; Nakata et al., 2006) however, pravastatin has been shown the increase insulin sensitivity in rats and thereby reducing insulin resistance. 
(Takagi et al., 2008) Additionally, muscle myopathy associated with statin use has also been suggested to increase the risk of new-onset diabetes. (Colbert \& Stone, 2012)

Similarly, antidepressants may also increase the risk of new-onset diabetes by changes in body weight (Zimmermann, Kraus, Himmerich, Schuld, \& Pollmächer, 2003) and modifications in the normal function of glucose homeostasis. (Carvalho et al., 2004; Fisfalen \& Hsiung, 2003; Levkovitz et al., 2007) Other mechanisms include impairment of insulin secretion and changes in insulin sensitivity due to high affinity of antidepressants towards muscarinic receptors. (Jindal, 2009) Although, no biological relationship exists between the use of inhaled corticosteroids, the use of oral corticosteroids has been shown to affect insulin resistance (Nielsen et al., 2004; Tappy et al., 1994) and reducing glucose uptake and thereby affecting glucose metabolism. (Rizza, Mandarino, \& Gerich, 1982) Indeed some studies have also suggested that inhaled corticosteroid use is linked with higher serum glucose levels. (Slatore, Bryson, \& Au, 2009) Therefore, ICS use may have similar effects that may lead to hyperglycemia.

\section{Medication Use and New-onset Diabetes: Existing Evidence}

\section{$\underline{\text { Antidepressants Use and New-onset Diabetes }}$}

Investigators from a large randomized controlled trial have reported that antidepressant users had higher likelihood of developing diabetes compared to non-users. (Rubin et al., 2008) However, further studies have not conclusively determined the role of antidepressants in causing new-onset diabetes. Some evidence also indicates that rather than antidepressants, presence of depression is associated with new-onset diabetes. (Sambamoorthi, Ma, Findley, \& Rust, 2012; Wilkins \& Sambamoorthi, 2011) The growing prevalence of depression among COPD patients and potential use of antidepressants with other therapies make them vulnerable to incident diabetes. Therefore, while examining potential novel therapies for COPD, it is important to understand its safety issues in terms of new-onset diabetes. 


\section{$\underline{\text { Inhaled Corticosteroids Use and New-Onset Diabetes }}$}

Inhaled corticosteroids (ICS) have also been evaluated with regards to development of type-2 diabetes among individuals with COPD due to the evidence related to hyperglycemia and incident diabetes associated with systemic corticosteroids.(Blackburn, Hux, \& Mamdani, 2002; O'Byrne et al., 2012; Suissa et al., 2010) It has been indicated that ICS levels in the systemic circulations are low and thus may not cause the risk of incident diabetes. However, the evidence regarding the risk of new-onset diabetes associated with ICS use is inconclusive.

A nested case-control study using the data from Quebec health insurance databases, Suissa et al reported that among 388,584 individuals with respiratory diseases, current ICS use was associated with $34 \%$ higher risk of incident diabetes compared to no current use of ICS (RR: 1.34; 95\% CI: 1.29-1.39). (Suissa et al., 2010) However, a major limitation of this study was the combined evaluation of asthma and COPD patients. (Suissa et al., 2010) It has been established, that the risk of incident diabetes is different among individuals with COPD than those with asthma. (Rana et al., 2004) Recently, a retrospective analysis of randomized controlled trials of budosenide, an ICS, indicated no statistically significant difference between users and non-users in terms of new-onset diabetes. (O'Byrne et al., 2012) Therefore the relationship between ICS use and new-onset diabetes remains uncertain. However, some studies have also reported higher blood glucose concentration with ICS use among individuals with diabetes. (Slatore et al., 2009) Therefore, while evaluating new-onset diabetes among individuals with COPD it is important to understand the role of ICS.

\section{Statins Use and New-onset Diabetes}

As per the USFDA labeling guidelines, statin medications may be linked with elevation of blood sugar levels (hyperglycemia) and risk of developing type-2-diabetes mellitus. (US FDA, 
2012) Investigators of several large scale randomized controlled trials have reported inconsistent findings. For example, analysis of data from the Justification for the Use of Statins in Prevention: an Intervention Trial Evaluating Rosuvastatin (JUPITER) trial revealed that among 17,802 individuals randomly assigned to rosuvastatin or placebo, those in the rosuvastatin group had significantly higher rates of physician-reported incident diabetes cases as compared to those in the placebo group (3.0\% vs. $2.4 \%$; $\mathrm{p}=0.01$ ). (Ridker et al., 2008) This trial included individuals with low cardiovascular risk but high levels of inflammatory mediator C-reactive protein, a characteristic of smokers with COPD. (Ridker et al., 2008) On the contrary, findings from the large scale randomized trial of pravastatin (WOSCOPS - West of Scotland Coronary Prevention Study) suggested protective effects of pravastatin in reducing the risk of new-onset diabetes. The researchers reported that among men aged between 45-64 years, individuals randomized on pravastatin therapy had $20 \%$ reduced hazard of developing new-onset diabetes as compared to individuals on placebo (HR: 0.70; 95\% CI: 0.50-0.99; $\mathrm{p}=0.042$ ). (Freeman et al., 2001)

These findings have raised substantial concerns regarding the use of statins. Therefore, many meta-analyses of statin trials have been conducted to better understand the effects of statins on new-onset diabetes. Even though, these meta-analyses have also yielded conflicting results, a closer examination of the results in a recent review suggests that the results from these studies are highly dependent on data from the WOSCOPS Study. (Colbert \& Stone, 2012) For example, meta-analysis of 5 statin trials by Coleman et al indicated that statin use is not associated with increased risk of incident diabetes (RR: 1.03; 95\% CI: 0.89-1.19). However, the sensitivity analysis results after removing data from the WOSCOPS study revealed an increased risk of incident diabetes (RR: 1.14; 95\% CI: 1.02-1.28). (Coleman, Reinhart, Kluger, \& White, 2008) Similarly, investigators of a meta-analysis conducted in 2009 which additionally included results 
from the JUPITER trial suggested that statin did not increase the risk of incident diabetes (RR: 1.06; 95\% CI: 0.93-1.25); however, removal of data from WOSCOPS study revealed an increased risk of incident diabetes with statin use as compared to placebo (RR: 1.13 ; $95 \%$ CI: 1.03-1.23). (Rajpathak et al., 2009) Recently, several other meta-analyses have been conducted that included data from additional randomized controlled trials. The findings from a metaanalysis conducted in 2010, which included 13 major trials' data indicated a slightly higher risk of incident diabetes among statin users compared to individuals on placebo (RR: 1.09; 95\% CI: 1.02-1.17). (Sattar et al., 2010) Subsequent meta-analyses have confirmed these findings. (Mills et al., 2011; Preiss et al., 2011)

These meta-analyses highlight the discrepancies in the findings related to statin use and new-onset diabetes. Moreover, the studies also raise an essential issue of studying the impact of individual statins as compared to collectively assessing the relationship between statin use and new-onset diabetes. Indeed, the subject of examining individual statin data has been emphasized in a recent review of literature on statin use and new-onset diabetes. (Colbert \& Stone, 2012)

Some clinical trials of specific statins have assessed the risk of developing new-onset diabetes with statin use. The results from trials that included Atorvastatin as investigational drug have shown that atorvastatin is associated with increased risk of new-onset diabetes when compared with placebo (Anglo-Scandinavian Cardiac Outcomes Trial (ASCOT) (Sever et al., 2003) and Stroke Prevention by Aggressive Reduction in Cholesterol Levels (SPARCL)) (Karam, Loney-Hutchinson, \& McFarlane, 2008) but not when compared with an active control (Treating to new targets trial (TNT) (LaRosa et al., 2005) and Incremental Decrease in End Points Through Aggressive Lipid Lowering (IDEAL) study). (Pedersen et al., 2005) As mentioned earlier, rosuvastatin use has been associated with increased risk of new-onset diabetes 
compared to placebo in the JUPITER trial; however, the Controlled Rosuvastatin Multinational Trial in Heart Failure (CORONA) (Kjekshus et al., 2007) study did not indicate higher risk of new-onset diabetes (RR: 1.13; 95\% CI: 0.86 - 1.50). Similarly, simvastatin trials have also not indicated any relationship between drug use and incident diabetes. By contrast, one pravastatin trial (WOSCOPS) (Freeman et al., 2001) has indicated protective effects of pravastatin terms of incident diabetes; whereas, another pravastatin trial concluded that there was not a statistically significant relationship between pravastatin use and incident diabetes.

Overall, based on the current understanding of medication use and new-onset diabetes suggests conflicting conclusions. Moreover, to date no study has examined the risk of new-onset diabetes associated with multiple medication use among individuals with COPD. Owing to the higher incidence of diabetes among individuals with COPD, it is important to comprehensively assess the safety of medications (new-onset diabetes) among individuals with COPD is required.

\section{Need for the Study}

The prevalence of diabetes has been increasing affecting over 18 million Americans.

(Geiss, 2012) Diabetes is a preventable epidemic which costs about $\$ 174$ billion in direct medical costs. A variety of avoidable risk factors are associated with new-onset diabetes such as multiple medication use. With the growing prevalence of multimorbidity, (Schnell et al., 2012) multiple medication use has become a reality (Anecchino et al., 2007) and it is essential to comprehensively evaluate the relationship between medication use and diabetes.

Recently, antidepressants, ICS and statin use have been studied with respect to development of diabetes. As the individuals with COPD have high prevalence of inflammationrelated multimorbidity, the use of these medications is very high among those with COPD. Furthermore, the use of statins is also being evaluated for its role in COPD management. While 
testing the effectiveness of statins as disease modifying therapy for individuals with COPD, it is important to examine any potential safety concerns.

There are several important reasons for assessing the risk of new-onset diabetes among individuals with COPD. COPD is linked with chronic systemic inflammation, which is being recognized as a new risk factor for the development of type-2 diabetes. (Festa, D'Agostino, Tracy, \& Haffner, 2002; Pradhan, Manson, Rifai, Buring, \& Ridker, 2001; Spranger et al., 2003) Tobacco smoking is associated with higher levels of inflammatory mediators which may lead to other inflammation related diseases. Indeed, several investigators have pointed out the impact of pro-inflammatory cytokines on the pathogenesis of insulin resistance and type-2 diabetes. (Festa et al., 2002; Hu, Meigs, Li, Rifai, \& Manson, 2004; Pradhan et al., 2001; Spranger et al., 2003) As the levels of these cytokines are elevated in individuals with COPD, the risk of developing type-2 diabetes may be more among individuals with COPD. (P. J. Barnes, 2000; Renauld, 2001) An analyses of data from prospective Nurses' Health Study indicated that among 103,614 females nurses, the risk of new-onset type-2 diabetes was significantly higher among those with COPD compared to those without COPD (RR: 1.8, 95\%: CI 1.1-2.8). (Rana et al., 2004) Due to higher risk of new-onset diabetes among COPD patients, we need to evaluate new-onset diabetes as an important safety measure for all the medications used by those with COPD.

This study provides important evidence regarding avoidable risk factors for new-onset diabetes. This study is timely because novel treatments such as statins are being evaluated for the management of COPD. Therefore, the findings from this study provide evidence about safety of statins in COPD as well as provide hypothesis for future randomized controlled trials.

Furthermore, our study gives important policy implications in terms of management of Medicaid beneficiaries with COPD. With the help of this study, we evaluate the impact of 
multiple medication use. Therefore, evidence regarding the adverse impact of management of cooccurring conditions may help in designing effective and tailored disease management programs for Medicaid beneficiaries with COPD. 


\section{CONCEPTUAL FRAMEWORK}

For the purposes of this study we used the conceptual framework delineating the determinants of health. This model describes several risk factors that lead to development of chronic diseases. Thus it can be considered as a chronic disease incidence model. Due to the nonavailability of certain factors specifically mentioned in the original model, we used a modified "determinants of health" model. The model that guided us in determining the risk factors associated with new-onset diabetes is mentioned in Figure 1 below.

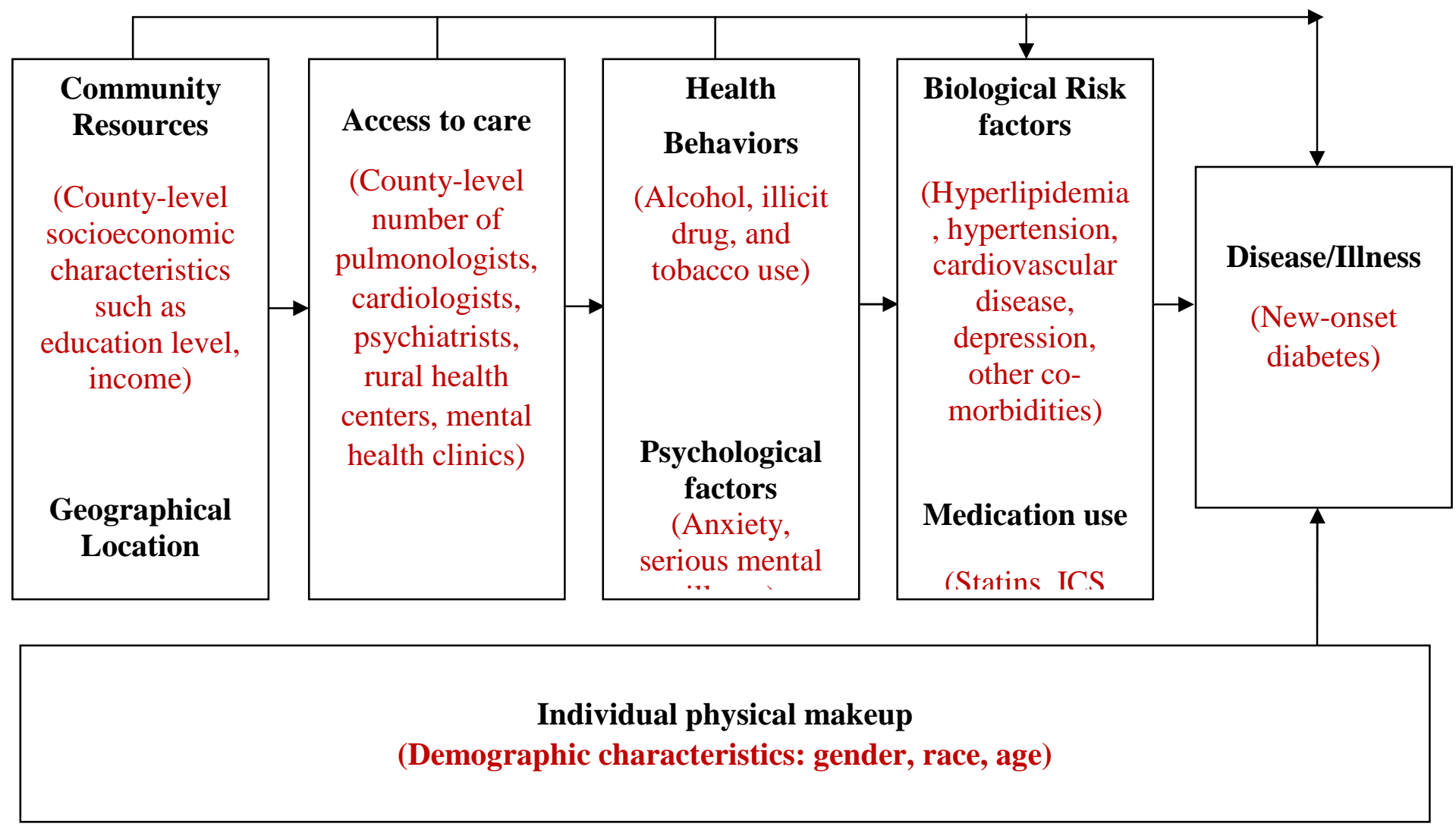

Figure 1: Conceptual Framework for Determinants of Health and Chronic disease incidence 
Figure 1 describes a comprehensive view of various pathways that can increase the risk of new-onset diabetes. This model was originally designed as a chronic disease incidence model. However, as our study population comprises of individuals with a chronic disease (COPD), we have adapted this model and operationalized based on our requirements. The Determinants of Health and Chronic Disease Incidence model describes the relationship between societal factors, access to care variables, individuals' health behavior, psychological factors and biological risk factors. Individuals' non-modifiable characteristics also play an important role in determining the biological conditions. In our study an additional factor needs to be considered that these individuals already have a chronic disease and thus are at a higher risk of other co-occurring conditions. The interplay between these factors causes disorders that require appropriate medical treatment. However, long term-use of therapeutic regimens may affect the normal bodily functions leading to further deterioration and causing illness (for example in this study we will be testing the association between statin use and new-onset diabetes).

\section{METHODS}

\section{Study design}

A retrospective longitudinal cohort design was used to determine the relationship between multiple medication use (antidepressants, ICS and statins) and new-onset diabetes. For the purposes of this study data from administrative claims of Medicaid enrollees obtained from the Medicaid Analytic eXtract (MAX) (2005-2008) provided by the Centers for Medicare and Medicaid Services (CMS) was used. Medicaid beneficiaries with newly-diagnosed COPD were identified between $01 / 01 / 2006$ to $12 / 31 / 2007$. The first date with any inpatient or outpatient claim of COPD diagnosis was considered as the index date. One year prior to the index date was considered as the baseline period which. Beneficiaries with COPD or diabetes during this period 
were excluded from the analyses. New-onset diabetes was identified during the follow-up period which was defined as 1 year after the index date.
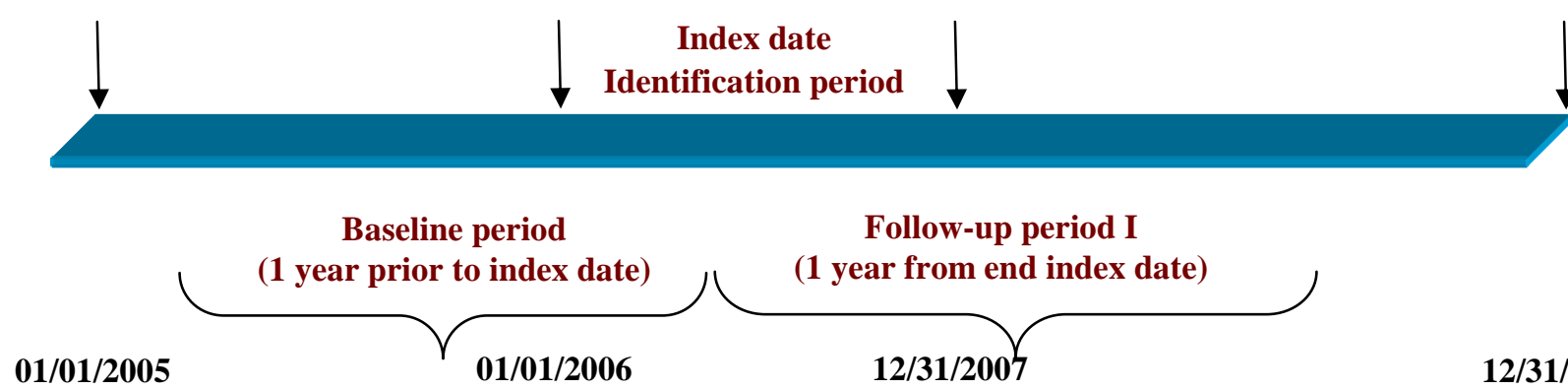

Follow-up period I

12/31/2007

$12 / 31 / 2008$

Figure: An example of description of study periods

\section{Data Source}

\section{Medicaid Analytic eXtract (MAX) (2005-2008)}

For the purposes of this study we utilized the data from the Medicaid analytic extract (MAX). The files provided in the MAX administrative claims data are prepared and produced by the Centers for Medicare and Medicaid Services with the help of Research and Data Assistance Center (ResDAC). The primary purpose of producing these files is to supply information regarding healthcare utilization of Medicaid beneficiaries to researchers and policy maker.

The MAX files contain person-level data which is obtained via Medicaid Statistical Information System (MSIS) in which all the states administering Medicaid have to supply the information for the Medicaid beneficiaries to the CMS. These person level files provided information about beneficiaries' eligibility and their healthcare utilization and payment information. Several initiatives are taken by the agencies to maintain and improve the quality of these datasets. (CMS, 2011; Hennessy, Leonard, Palumbo, Newcomb, \& Bilker, 2007) We received separate files from CMS which were linked based on beneficiaries' unique identification numbers. These files include enrollment ("personal summary"), inpatient and outpatient medical claims, and pharmacy claims files for beneficiaries. 
The personal summary file provided information on eligibility, demographics (age, gender, and race), managed care enrollment, utilization summary and Medicaid payments. Three other files were used to capture the information regarding beneficiaries' fee-for-service claims. From the "Other therapy" file we collected information regarding claims for Medicaid services provided at an outpatient level such as clinic services, physician services, home health and lab services. Enrollees' information regarding services provided during hospitalizations was obtained from the "Inpatient file". All the information regarding pharmacy or drug services was captured from the "Prescription Drug" file. This file included information regarding utilization of prescription drugs by Medicaid beneficiaries such as date of prescription filled, days of supply, national drug code (NDC).

We used data for beneficiaries residing in the following states: New York (NY), Texas (TX), Illinois (IL), and California (CA). The primary reason behind using this data was to obtain programs with lower Medicaid-managed care penetration rates so that more utilization characteristics would be captured. This provided us the ability to obtain maximum number of beneficiaries with fee-for-service enrollments.

\section{Area Resource File $(A R F)$ :}

In addition to all the demographic characteristics and utilization variables obtained from the MAX files, we also determined each Medicaid beneficiary's contextual county level variables using the Area Resource File $(\boldsymbol{A R F})$. ARF is a comprehensive county-level dataset compiled by the Health Resources Service Administration's Bureau of Health Professions. ARF contains more than 6000 variables providing information about nation's counties. The information contained within ARF includes type of health facilities in the various counties, number and type health professions, resource scarcity measures, health status, economic activity, health training programs, and socioeconomic and environmental characteristics. These files also 
include county codes and descriptors that allow its linkage with several secondary datasets such as MAX. The type of variables that were obtained from ARF included density of pulmonologist in beneficiary's residing county. We used the county codes and state information to link MAX files with 2008 ARF file. All the ARF variables were obtained from the baseline period.

\section{Study Population}

\section{Medicaid Beneficiaries with newly-diagnosed COPD}

Medicaid beneficiaries with newly-diagnosed COPD were identified using MAX IP and OT files (physician office visits claims only). Baseline period was used to identify Medicaid Beneficiaries with newly-diagnosed COPD. Individuals with at least one inpatient visit or two 14 days apart outpatient visits (obtained using type of service codes) for COPD based on International Classification of Diseases, Ninth Revision, Clinical Modification (ICD-9-CM) codes for chronic bronchitis (491.xx), emphysema (492.xx), or unspecified chronic airway obstruction (496.xx) were considered to have diagnosed COPD. These diagnosis codes have been utilized in prior published research (codes 491.xx, 492.xx and 496.xx) to identify COPD and evaluate medical treatment and health outcomes among individuals with COPD. (Dalal, Shah, D'Souza, Chaudhari, \& Crater, 2012; Halpern et al., 2011; Make, Dutro, Paulose-Ram, Marton, \& Mapel, 2012) Indeed, sensitivity and specificity of using ICD-9-CM codes to identify patients with COPD has been established. (Cooke et al., 2011; A. S. Gershon et al., 2009) A study conducted by Gershon et al reported that identifying COPD using 1 or more ambulatory claims and/or 1 or more hospitalizations for COPD resulted in a sensitivity of $85.0 \%$ (95\% CI: 77.0 to 91.0$)$ and a specificity of $78.4 \%$ (95\% CI: 73.6 to 82.7$)$. However, we will be used 1 inpatient or 2 outpatient claims to identify individuals with COPD in order to increase the specificity of the algorithm. 
Other inclusion criteria will included: a) no COPD diagnoses during baseline period; b) no diabetes diagnoses (ICD-9-CM: 250.x2) during the baseline period; c) 40-64 years (among young adults this age group is at highest risk of COPD); d) continuous eligibility during the baseline and follow-up period; e) no dual Medicaid/Medicare coverage (dual eligibility will represent high-cost and severe beneficiaries); f) enrolled in fee-for-service plans throughout the study observation period; g) Alive during the study observation period; and use of services (inpatient or outpatient).

\section{Dependent Variable (New-onset diabetes)}

New-onset diabetes was identified in the follow-up period for beneficiaries who were diabetes free in the year prior to baseline year. Any Medicaid beneficiary who had at least 1 inpatient or 2 outpatient claims for diabetes based on ICD-9-CM diagnosis (250.x2) were considered as having new-onset diabetes

\section{Key Independent variables}

\section{Multiple Medication Use}

Antidepressants, ICS and statins were identified using National Drug Codes (NDC) recorded in pharmacy claims. Although, identifying medication use is difficult using administrative claims data, any receipt of these medications was considered as use of medications.

3. Any Statin use (Yes/No)

Adults with at least one prescription of statin during the baseline period were considered as statin users.

4. Statin Type (Hydrophilic vs. Lipophilic Statins)

Statins were further grouped based upon their chemical nature. Statins with lipophilic characteristics (Atorvastatin, Cerivastatin, Fluvastatin, Lovastatin, Pitavastatin, and Simvastatin) 
were classified as lipophilic statins and those with hydrophilic properties (Pravastatin, Rosuvastatin) were grouped as hydrophilic statins.

5. Any ICS use (Yes/No)

ICS use was identified during the follow-up period as our population consisted of individuals with newly-diagnosed COPD and thus we did not have many cases with ICS use in the baseline period.

6. Any Antidepressants use (Yes/No)

Antidepressant prescriptions were identified during baseline.

\section{Long-term use (Antidepressants and Statins)}

Medicaid beneficiaries with newly-diagnosed COPD having $\geq 120$ days of supply for these medications during the baseline period were considered as long-term users. Those with <120 days of supply were categorized as short-term users and those without any use in the baseline period were considered as non-users.

\section{Inflammation-related multimorbidity:}

We created indicator variables for the presence of arthritis, CVD, depression, and osteoporosis using the International Classification of Diagnosis $-9^{\text {th }}$ Revision- Clinical Modification (ICD-9-CM) codes. Any Medicaid beneficiary with COPD having greater than or equal to one inpatient or one outpatient visits for these conditions were considered as having the disease. In addition indicator variables were created for inflammation-related physical conditions and inflammation-related mental condition. Any Medicaid beneficiary having either of the following conditions: arthritis, CVD, and osteoporosis were considered as having inflammationrelated physical condition. Similarly, any individual with COPD having depression were considered as having inflammation-related mental condition. 


\section{Operational definition:}

Medicaid beneficiaries included in our study population were categorized into one of the four multimorbidity groups as mentioned below:

9) COPD + Inflammation-related Physical Conditions + Inflammation-related Mental Condition (i.e. depression)

10) COPD + Inflammation-related Physical Conditions

11) COPD + Inflammation-related Mental Condition (i.e. depression)

12) COPD only (without any chronic conditions).

\section{Other Independent Variables}

1. Individual Physical Makeup

These variables include demographic characteristics such as age (will be grouped based on data), gender (female/male), race/ethnicity (Whites, African- Americans and Others).

2. Community resources

Community resources consist of variables regarding socioeconomic makeup of the society in which the Medicaid beneficiaries reside. These include variables such as median income at county level and county level education status. All the county level information was is obtained from the ARF. Variables included in this study were: Quartiles for density of above high school education, unemployment and poverty were created. Density of these county level characteristics was calculated by dividing total number of each characteristic by the total county population. This density was further converted into per 1000 people by multiplying 1000 with the density. Geographical Location

Metro status was ascertained for each Medicaid beneficiary. All the Medicaid beneficiaries with COPD were categorized into metropolitan residents and non-metropolitan residents based county level information obtained from the ARF.

3. Access to care variables 
Access to care variables was identified as county level characteristics from the ARF. These characteristics included density of primary care providers in the county (Quartiles); density of specialist providers (Quartiles), primary care shortage area (yes/no) mental health shortage area (yes/no); hospital beds density (yes/no) pulmonologist density (high/low) and cardiologist density (yes/no)

\section{Health behavior}

Alcohol, illicit drug (substance abuse) and tobacco use were identified as health behaviors. These variables were identified using ICD-9-CM codes from Medicaid claims. ICD-9-CM codes for these conditions were obtained from AHRQ's clinical classification software.

5. Psychological Factor

Serious mental illness among Medicaid beneficiaries with COPD will be determined as psychological risk factor. Conditions included within serious mental illness were schizophrenia, bipolar disorder and psychoses. ICD-9-CM codes for these conditions were obtained from AHRQ's clinical classification software.

\section{Biological Risk Factors}

Biological risk factor variables includes inflammation-related multimorbidity and other co-occurring conditions identified as number of other clinical conditions (categorized into: a) None, b) 1-3; c) 4-6; d >6 conditions. All these conditions were identified using ICD-9-CM codes obtained from AHRQ's clinical classification software.

\section{Statistical Analyses}

\section{Bivariate Analyses}

Chi-square tests of independence were used to determine subgroup differences in antidepressant, ICS and statin prescriptions and new-onset diabetes. Results from unadjusted 
logistic regressions are also presented for better understanding of bivariate relationship between specific medication use categories and new-onset diabetes.

\section{$\underline{\text { Multivariate Analyses }}$}

We used multivariable logistic regression to examine the relationship between any antidepressants, ICS and statins use and new-onset diabetes after controlling for comprehensive set of risk factors as described in the Methods section. SAS v 9.3 (SAS Institute, Cary, NC) was used for all the analyses.

\section{RESULTS}

Overall, 6.3\% ( $\mathrm{n}=967)$ Medicaid beneficiaries with newly-diagnosed COPD had newonset diabetes during the follow-up period. In the study population, $47.8 \%$ individuals received antidepressants, $24.3 \%$ received statins and $42.9 \%$ received ICS. The bivariate differences in medication use by selected beneficiary characteristics are presented in Table 1.

\section{Antidepressants, ICS, Statins, and New-Onset Diabetes}

Table 2 describes the unadjusted and adjusted relationship between any antidepressants, statins and ICS use and new-onset diabetes. Rates of new-onset diabetes were not significantly different between antidepressants users and non-users (6.5\% vs. 6.2\%). However, beneficiaries with statin use had significantly higher rates of new-onset diabetes compared to those without statin use (9.2\% vs. 5.4\%; p<0.001). Similarly, individuals with ICS use had significantly higher rates of new-onset diabetes compared to those without ICS use (7.4\% vs. 5.5\%; p<0.001). After controlling for baseline characteristics beneficiaries who received statins and ICS were $48 \%$

(AOR: 1.48; 95\% CI: 1.27, 1.72) and 23\% (AOR: 1.23; 95\% CI: AOR: 1.07, 1.47) more likely to have new-onset diabetes as compared to their counterparts without these medications. 
In order to assess the relationship between combined medication categories and newonset diabetes, we created a variable with the following categories: 1) all three medications; 2) antidepressant/statins; 3) antidepressants/ICS; 4) statins/ICS; 5) statins only; 6) ICS only; and 7) none of these medications. Findings from bivariate and multivariate analyses with combined medication categories are presented in Table 3. Adults with all three medications were significantly more likely to have new-onset diabetes (AOR: 1.56 ; 95\% CI: 1.18, 2.05) compared to those without any of these three medications. Adults who used statins combination with antidepressants and ICS or only ICS were more likely to have new-onset diabetes compared to those without any medications. Other combinations which did not include statins were not associated with new-onset diabetes.

\section{Type of Statins and New-Onset Diabetes}

The findings from analyses on new-onset diabetes with type of statins are presented in Table 4. As number of non-elderly Medicaid beneficiaries with new-onset diabetes in the "both hydrophilic and lipophilic" was very small $(\mathrm{n}=9)$, we removed individuals with "both hydrophilic and lipophilic" from the analyses. Therefore, the analyses on type of statins included 15,128 Medicaid beneficiaries with newly-diagnosed COPD who did not receive both types of statins. We observed no statistically significant relationships between types of statins and newonset diabetes.

\section{Duration of Antidepressants, Statin Use and New-Onset Diabetes}

Duration of medication use (antidepressants) did not have a statistically significant association with new-onset diabetes. Adults with both long-term (AOR: 1.44, 95\% CI: 1.22, $1.70)$ and short-term statin use (AOR: $1.58,95 \%$ CI: $1.25,1.99)$ were more likely to have newonset diabetes compared to those without any statin use. 


\section{Secondary Analysis (Controlling for Selection Bias in Statin Use)}

As statin use was associated with new-onset diabetes, we also controlled for selection bias in unobserved variables. For example, statin users may be different from non-users in terms of genetic risk factors or presence of pre-diabetic condition. To control for this selection bias, we conducted secondary analyses with instrumental variable regression. We used countylevel variables obtained from the ARF including poverty status (categorized into quartiles) and density of pulmonologist in a county in selection equation that predicted the presence/absence of statin use. The model on new-onset diabetes included all independent variables mentioned in the methods section. This analysis was conducted using STATA v. 11. The instrumental variable approach revealed that statin use was not associated with new-onset diabetes (parameter estimate $=0.15 ; \mathrm{p}=0.613)$.

\section{DISCUSSION}

Using a retrospective longitudinal dynamic cohort design, we examined the relationship between commonly used medications such as antidepressants, ICS, and statins and new-onset diabetes among Medicaid beneficiaries with newly-diagnosed COPD. For the purposes of these analyses we used Medicaid claims data from multiple years (2005-2008). To the best of our knowledge, this is the first observational study to examine this relationship using Medicaid claims data of non-elderly adults with newly-diagnosed COPD. Therefore, we cannot directly compare the study results with any prior studies.

In our study, after controlling for baseline characteristics, we did not observe a statistically significant association between antidepressant use and new-onset diabetes. These results are consistent with findings using data from general population (not specific to COPD). For example, no significant association between antidepressants and new-onset diabetes was 
found in previous studies (Sambamoorthi, Ma, Findley, \& Rust, 2012; Wilkins \& Sambamoorthi, 2011; Bhattacharrya R, 2014). Recently, a review on glucose metabolism and antidepressant medications has suggested that the relationship between antidepressant use and new-onset diabetes may vary by type of antidepressants. Therefore, it is plausible that deleterious effects of non-adrenergic antidepressants are negated by beneficial effects of mono-amine oxidase inhibitors with regards to glucose metabolism. (Hennings JM, 2012) Therefore, further analyses are required between type of antidepressant use and new-onset diabetes. Moreover, to draw conclusive evidence, randomized controlled trials among those with COPD are required to determine the safety of antidepressant in terms of new-onset diabetes.

As statins are being investigated as novel therapeutic agents among individuals with COPD, we also examined the relationship between statins and new-onset diabetes. Our primary analyses revealed that adults who had any statin use were more likely to have new-onset diabetes compared to those without statins. However, once we controlled for selection bias due to unobserved characteristics the statistically significant association disappeared. Although, no specific study has examined the risk of new-onset diabetes among COPD patients, randomized controlled trials and meta-analyses have yielded conflicting results. A meta-analysis including the data from WOSCOPS study has shown that statin use did not increase the risk of new-onset diabetes. (Rajpathak et al., 2009) Whereas, another set of analyses have reported that statin use is associated with greater risk of new-onset diabetes. (Sattar et al., 2010; Mills et al., 2011; Preiss et al., 2011) Therefore, more research is required in high-risk populations such as in our study to strengthen the knowledge base of the safety of these medications. As statins are being investigated for their use in COPD, clinical trials should include new-onset diabetes as a safety outcome. 
We also tested for the relationship between ICS and new-onset diabetes among newlydiagnosed COPD patients. However, as our population consisted of only newly-diagnosed cases, we could only test for exposure to ICS and new-onset diabetes in the same follow-up period. We did not find an association between ICS and new-onset diabetes when we combined all three medications and derived a combination variable with 7 categories. Our findings are different from those observed in a retrospective cohort study using Quebec Health Insurance database, which included patients with any respiratory disease. (Suissa, 2010) The inconsistent findings could be due to differences in study populations.

Overall, the findings from our study indicated that after controlling for baseline characteristics and selection bias, medication use (antidepressants, ICS and statins) was not associated with increased risk of new-onset diabetes when compared to those who did not receive any of these medications. However, as the prevalence of diabetes is increasing and individuals with COPD are at a greater risk of having new-onset diabetes due to systemic inflammation, continuous glucose monitoring for COPD patients may be needed. In future, long-term observational studies and randomized controlled trials need to be conducted to evaluate the safety of above mentioned medications.

\section{STRENGTHS AND LIMITATIONS}

There are many advantages to this study. Use of Medicaid claims data enabled us to track individuals over two years and adopt a longitudinal design. Clinical diagnoses with ICD9 codes provided information on medical conditions. Prescription drug claims facilitated identification of specific drugs and the precise time in which they were prescribed enabling us to calculate duration of medication use. The study also employed statistical tools that controlled for selection bias due to unobserved characteristics. 
An important limitation of this study is lack of generalizability, as we included only feefor-service claims of Medicaid beneficiaries living in four states. We only observed prescriptions for specific medications and cannot determine use of these medications. Furthermore, unavailability of laboratory data limited our ability to control for important confounders such as cholesterol levels and baseline blood glucose levels. Additionally we did not have the data for body mass index which is an important predictor of new-onset diabetes. 


\section{REFERENCES}

Anecchino, C., Rossi, E., Fanizza, C., De Rosa, M., Tognoni, G., \& Romero, M. (2007).

Prevalence of chronic obstructive pulmonary disease and pattern of comorbidities in a general population. International Journal of Chronic Obstructive Pulmonary Disease, 2(4), 567-574.

Barnes, P. J. (2000). Chronic obstructive pulmonary disease. The New England Journal of Medicine, 343(4), 269-280.

Barnes, P. J., \& Celli, B. R. (2009). Systemic manifestations and comorbidities of COPD. The European Respiratory Journal, 33(5), 1165-1185. doi:10.1183/09031936.00128008; $10.1183 / 09031936.00128008$

Bhattacharjee, S., Bhattacharya, R., Kelley, G. A., \& Sambamoorthi, U. (2013). Antidepressant use and new-onset diabetes: A systematic review and meta-analysis. Diabetes/metabolism Research and Reviews, 29(4), 273-284. doi:10.1002/dmrr.2393

Bhattacharya R, Ajmera M, Bhattacharjee S, Sambamoorthi U. Use of antidepressants and statins and short-term risk of new-onset diabetes among high risk adults. Diabetes Res Clin Pract. 2014 Jun 4. pii: S0168-8227(14)00199-5. doi: 10.1016/j.diabres.2014.04.016. [Epub ahead of print]

Blackburn, D., Hux, J., \& Mamdani, M. (2002). Quantification of the risk of corticosteroidinduced diabetes mellitus among the elderly. Journal of General Internal Medicine, 17(9), 717-720.

Carvalho, F., Barros, D., Silva, J., Rezende, E., Soares, M., Fregoneze, J., \& De Castro, e. S. (2004). Hyperglycemia induced by acute central fluoxetine administration: Role of the central CRH system and 5-HT3 receptors. Neuropeptides, 38(2-3), 98-105.

Chamberlain, L. H. (2001). Inhibition of isoprenoid biosynthesis causes insulin resistance in 3T3-L1 adipocytes. FEBS Letters, 507(3), 357-361.

CMS. (2011). Medicaid analytic eXtract (MAX) general information. (https://www.cms.gov/MedicaidDataSourcesGenInfo/07_MAXGeneralInformation.asp). Baltimore, MD: 50. Centers for Medicare and Medicaid Services, US Department of Health and Human Services. 
Colbert, J. D., \& Stone, J. A. (2012). Statin use and the risk of incident diabetes mellitus: A review of the literature. The Canadian Journal of Cardiology, 28(5), 581-589.

doi:10.1016/j.cjca.2012.03.021

Coleman, C. I., Reinhart, K., Kluger, J., \& White, C. M. (2008). The effect of statins on the development of new-onset type 2 diabetes: A meta-analysis of randomized controlled trials. Current Medical Research and Opinion, 24(5), 1359-1362.

doi:10.1185/030079908X292029

Cooke, C. R., Joo, M. J., Anderson, S. M., Lee, T. A., Udris, E. M., Johnson, E., \& Au, D. H. (2011). The validity of using ICD-9 codes and pharmacy records to identify patients with chronic obstructive pulmonary disease. BMC Health Services Research, 11, 37-37. doi:10.1186/1472-6963-11-37

Dalal, A. A., Shah, M., D'Souza, A.,O., Chaudhari, S., \& Crater, G. (2012). Clinical and economic outcomes for patients initiating fluticasone propionate/salmeterol combination therapy $(250 / 50 \mathrm{mcg}$ ) versus anticholinergics in a comorbid COPD/depression population. International Journal of Chronic Obstructive Pulmonary Disease, 7, 11-19. doi:10.2147/COPD.S27846

Fabbri, L. M., \& Rabe, K. F. (2007). From COPD to chronic systemic inflammatory syndrome? Lancet, 370(9589), 797-799.

Festa, A., D'Agostino, R., Jr, Tracy, R. P., \& Haffner, S. M. (2002). Elevated levels of acutephase proteins and plasminogen activator inhibitor-1 predict the development of type 2 diabetes: The insulin resistance atherosclerosis study. Diabetes, 51(4), 1131-1137.

Fisfalen, M. E., \& Hsiung, R. C. (2003). Glucose dysregulation and mirtazapine-induced weight gain. The American Journal of Psychiatry, 160(4), 797-797.

Freeman, D. J., Norrie, J., Sattar, N., Neely, R. D., Cobbe, S. M., Ford, I., . . Gaw, A. (2001). Pravastatin and the development of diabetes mellitus: Evidence for a protective treatment effect in the west of scotland coronary prevention study. Circulation, 103(3), 357-362.

Geiss, L. S. (2012). Increasing prevalence of diagnosed diabetes - united states and puerto rico, 1995-2010 . Retrieved June 15, 2013, from http://www.cdc.gov/mmwr/preview/mmwrhtml/mm6145a4.htm

Gershon, A. S., Wang, C., Guan, J., Vasilevska-Ristovska, J., Cicutto, L., \& To, T. (2009). Identifying individuals with physcian diagnosed COPD in health administrative databases. COPD, 6(5), 388-394. 
Górska, K., Maskey-Warzechowska, M., \& Krenke, R. (2010). Airway inflammation in chronic obstructive pulmonary disease. United States: Lippincott Williams \& Wilkins. doi:10.1097/MCP.0b013e3283341ba0

Halpern, R., Baker, C. L., Su, J., Woodruff, K. B., Paulose-Ram, R., Porter, V., \& Shah, H. (2011). Outcomes associated with initiation of tiotropium or fluticasone/salmeterol in patients with chronic obstructive pulmonary disease. Patient Preference and Adherence, 5, 375-388. doi:10.2147/PPA.S19991

Hennessy, S., Leonard, C. E., Palumbo, C. M., Newcomb, C., \& Bilker, W. B. (2007). Quality of medicaid and medicare data obtained through centers for medicare and medicaid services (CMS). Medical Care, 45(12), 1216-1220. doi:10.1097/MLR.0b013e318148435a

Hennings JM, Schaaf L, Fulda S. Glucose metabolism and antidepressant medication. Curr Pharm Des 2012;18:5900-19

Hu, F. B., Meigs, J. B., Li, T. Y., Rifai, N., \& Manson, J. E. (2004). Inflammatory markers and risk of developing type 2 diabetes in women. Diabetes, 53(3), 693-700.

Jindal, R. D. (2009). Long-term antidepressant use and risk for diabetes: Cause for concern and optimism. The American Journal of Psychiatry, 166(9), 1065-1066.

doi:10.1176/appi.ajp.2009.09050629

Kanda, M., Satoh, K., \& Ichihara, K. (2003). Effects of atorvastatin and pravastatin on glucose tolerance in diabetic rats mildly induced by streptozotocin. Biological \& Pharmaceutical Bulletin, 26(12), 1681-1684.

Karam, J. G., Loney-Hutchinson, L., \& McFarlane, S. I. (2008). High-dose atorvastatin after stroke or transient ischemic attack: The stroke prevention by aggressive reduction in cholesterol levels (SPARCL) investigators. Journal of the Cardiometabolic Syndrome, 3(1), 68-69.

Kjekshus, J., Apetrei, E., Barrios, V., Böhm, M., Cleland, J. G. F., Cornel, J. H., . . Wikstrand, J. (2007). Rosuvastatin in older patients with systolic heart failure. The New England Journal of Medicine, 357(22), 2248-2261.

Koh, K. K., Sakuma, I., \& Quon, M. J. (2011). Differential metabolic effects of distinct statins. Atherosclerosis, 215(1), 1-8. doi:10.1016/j.atherosclerosis.2010.10.036

LaRosa, J. C., Grundy, S. M., Waters, D. D., Shear, C., Barter, P., Fruchart, J., . . Wenger, N. K. (2005). Intensive lipid lowering with atorvastatin in patients with stable coronary disease. The New England Journal of Medicine, 352(14), 1425-1435. 
Lehr, P. S. (2012). Global markets for asthma and COPD drugs. ().BCC Research.

Levkovitz, Y., Ben-Shushan, G., Hershkovitz, A., Isaac, R., Gil-Ad, I., Shvartsman, D., . . Zick, Y. (2007). Antidepressants induce cellular insulin resistance by activation of IRS-1 kinases. Molecular and Cellular Neurosciences, 36(3), 305-312.

Make, B., Dutro, M. P., Paulose-Ram, R., Marton, J. P., \& Mapel, D. W. (2012). Undertreatment of COPD: A retrospective analysis of US managed care and medicare patients. International Journal of Chronic Obstructive Pulmonary Disease, 7, 1-9. doi:10.2147/COPD.S27032

Mann, D., Reynolds, K., Smith, D., \& Muntner, P. (2008). Trends in statin use and low-density lipoprotein cholesterol levels among US adults: Impact of the 2001 national cholesterol education program guidelines. The Annals of Pharmacotherapy, 42(9), 1208-1215. doi:10.1345/aph.1L181

Mills, E. J., Wu, P., Chong, G., Ghement, I., Singh, S., Akl, E. A., . . Briel, M. (2011). Efficacy and safety of statin treatment for cardiovascular disease: A network meta-analysis of 170,255 patients from 76 randomized trials. QJM: Monthly Journal of the Association of Physicians, 104(2), 109-124. doi:10.1093/qjmed/hcq165

Mojtabai, R., \& Olfson, M. (2011). Proportion of antidepressants prescribed without a psychiatric diagnosis is growing. Health Affairs (Project Hope), 30(8), 1434-1442. doi:10.1377/hlthaff.2010.1024

Nakata, M., Nagasaka, S., Kusaka, I., Matsuoka, H., Ishibashi, S., \& Yada, T. (2006). Effects of statins on the adipocyte maturation and expression of glucose transporter 4 (SLC2A4): Implications in glycaemic control. Diabetologia, 49(8), 1881-1892.

Nielsen, M. F., Caumo, A., Chandramouli, V., Schumann, W. C., Cobelli, C., Landau, B. R., . . Schmitz, O. (2004). Impaired basal glucose effectiveness but unaltered fasting glucose release and gluconeogenesis during short-term hypercortisolemia in healthy subjects. American Journal of Physiology.Endocrinology and Metabolism, 286(1), E102-E110.

O'Byrne, ,P.M., Rennard, S., Gerstein, H., Radner, F., Peterson, S., Lindberg, B., . . Sin, D. D. (2012). Risk of new onset diabetes mellitus in patients with asthma or COPD taking inhaled corticosteroids. Respiratory Medicine, 106(11), 1487-1493. doi:10.1016/j.rmed.2012.07.011

Olfson, M., \& Marcus, S. C. (2009). National patterns in antidepressant medication treatment. Archives of General Psychiatry, 66(8), 848-856. doi:10.1001/archgenpsychiatry.2009.81 
Paulose-Ram, R., Safran, M. A., Jonas, B. S., Gu, Q., \& Orwig, D. (2007). Trends in psychotropic medication use among U.S. adults. Pharmacoepidemiology and Drug Safety, $16(5), 560-570$.

Pedersen, T. R., Faergeman, O., Kastelein, J. J. P., Olsson, A. G., Tikkanen, M. J., Holme, I., . . . Tsai, J. (2005). High-dose atorvastatin vs usual-dose simvastatin for secondary prevention after myocardial infarction: The IDEAL study: A randomized controlled trial. JAMA: The Journal of the American Medical Association, 294(19), 2437-2445.

Pradhan, A. D., Manson, J. E., Rifai, N., Buring, J. E., \& Ridker, P. M. (2001). C-reactive protein, interleukin 6, and risk of developing type 2 diabetes mellitus. JAMA: The Journal of the American Medical Association, 286(3), 327-334.

Preiss, D., Seshasai, S. R. K., Welsh, P., Murphy, S. A., Ho, J. E., Waters, D. D., . . Ray, K. K. (2011). Risk of incident diabetes with intensive-dose compared with moderate-dose statin therapy: A meta-analysis. JAMA: The Journal of the American Medical Association, 305(24), 2556-2564. doi:10.1001/jama.2011.860

Rabe, K. F., Hurd, S., Anzueto, A., Barnes, P. J., Buist, S. A., Calverley, P., . . Zielinski, J. (2007). Global strategy for the diagnosis, management, and prevention of chronic obstructive pulmonary disease: GOLD executive summary. American Journal of Respiratory and Critical Care Medicine, 176(6), 532-555.

Rajpathak, S. N., Kumbhani, D. J., Crandall, J., Barzilai, N., Alderman, M., \& Ridker, P. M. (2009). Statin therapy and risk of developing type 2 diabetes: A meta-analysis. Diabetes Care, 32(10), 1924-1929. doi:10.2337/dc09-0738

Rana, J. S., Mittleman, M. A., Sheikh, J., Hu, F. B., Manson, J. E., Colditz, G. A., . . Camargo,Carlos A.,Jr. (2004). Chronic obstructive pulmonary disease, asthma, and risk of type 2 diabetes in women. Diabetes Care, 27(10), 2478-2484.

Renauld, J. C. (2001). New insights into the role of cytokines in asthma. Journal of Clinical Pathology, 54(8), 577-589.

Ridker, P. M., Danielson, E., Fonseca, F. A. H., Genest, J., Gotto,Antonio M.,Jr, Kastelein, J. J. P., . . Glynn, R. J. (2008). Rosuvastatin to prevent vascular events in men and women with elevated C-reactive protein. The New England Journal of Medicine, 359(21), 2195-2207. doi:10.1056/NEJMoa0807646

Rizza, R. A., Mandarino, L. J., \& Gerich, J. E. (1982). Cortisol-induced insulin resistance in man: Impaired suppression of glucose production and stimulation of glucose utilization due 
to a postreceptor detect of insulin action. The Journal of Clinical Endocrinology and Metabolism, 54(1), 131-138.

Rubin, R. R., Ma, Y., Marrero, D. G., Peyrot, M., Barrett-Connor, E., Kahn, S. E., . . Knowler, W. C. (2008). Elevated depression symptoms, antidepressant medicine use, and risk of developing diabetes during the diabetes prevention program. Diabetes Care, 31(3), 420-426.

Sambamoorthi, U., Ma, Y., Findley, P. A., \& Rust, G. (2012). Antidepressant use, depression, and new onset diabetes among elderly medicare beneficiaries. Journal of Diabetes,

Sattar, N., Preiss, D., Murray, H. M., Welsh, P., Buckley, B. M., de Craen, A.,J.M., . . Ford, I. (2010). Statins and risk of incident diabetes: A collaborative meta-analysis of randomised statin trials. Lancet, 375(9716), 735-742. doi:10.1016/S0140-6736(09)61965-6

Schnell, K., Weiss, C. O., Lee, T., Krishnan, J. A., Leff, B., Wolff, J. L., \& Boyd, C. (2012). The prevalence of clinically-relevant comorbid conditions in patients with physician-diagnosed COPD: A cross-sectional study using data from NHANES 1999-2008. BMC Pulmonary Medicine, 12, 26-26. doi:10.1186/1471-2466-12-26

Sever, P. S., Dahlöf, B., Poulter, N. R., Wedel, H., Beevers, G., Caulfield, M., . . Ostergren, J. (2003). Prevention of coronary and stroke events with atorvastatin in hypertensive patients who have average or lower-than-average cholesterol concentrations, in the angloscandinavian cardiac outcomes trial--lipid lowering arm (ASCOT-LLA): A multicentre randomised controlled trial. Lancet, 361(9364), 1149-1158.

Slatore, C. G., Bryson, C. L., \& Au, D. H. (2009). The association of inhaled corticosteroid use with serum glucose concentration in a large cohort. The American Journal of Medicine, 122(5), 472-478. doi:10.1016/j.amjmed.2008.09.048

Spranger, J., Kroke, A., Möhlig, M., Hoffmann, K., Bergmann, M. M., Ristow, M., . . Pfeiffer, A. F. H. (2003). Inflammatory cytokines and the risk to develop type 2 diabetes: Results of the prospective population-based european prospective investigation into cancer and nutrition (EPIC)-potsdam study. Diabetes, 52(3), 812-817.

Suissa, S., Kezouh, A., \& Ernst, P. (2010). Inhaled corticosteroids and the risks of diabetes onset and progression. The American Journal of Medicine, 123(11), 1001-1006.

doi:10.1016/j.amjmed.2010.06.019

Takagi, T., Matsuda, M., Abe, M., Kobayashi, H., Fukuhara, A., Komuro, R., . . Shimomura, I. (2008). Effect of pravastatin on the development of diabetes and adiponectin production. Atherosclerosis, 196(1), 114-121. 
Tappy, L., Randin, D., Vollenweider, P., Vollenweider, L., Paquot, N., Scherrer, U., . . Jéquier, E. (1994). Mechanisms of dexamethasone-induced insulin resistance in healthy humans. The Journal of Clinical Endocrinology and Metabolism, 79(4), 1063-1069.

US FDA. (2012). FDA announces safety changes in labeling for some cholesterol-lowering drugs. . Retrieved June 15, 2013, from http://www.fda.gov/NewsEvents/Newsroom/PressAnnouncements/ucm293623.htm

Wilkins, T. L., \& Sambamoorthi, U. (2011). Antidepressant use, depression, lifestyle factors, and new-onset diabetes. International Clinical Psychopharmacology, 26(3), 159-168. doi:10.1097/YIC.0b013e328342ce31

Zimmermann, U., Kraus, T., Himmerich, H., Schuld, A., \& Pollmächer, T. (2003). Epidemiology, implications and mechanisms underlying drug-induced weight gain in psychiatric patients. Journal of Psychiatric Research, 37(3), 193-220. 


\section{TABLES}

\begin{tabular}{|c|c|c|c|c|c|c|c|c|c|}
\hline \multicolumn{10}{|c|}{$\begin{array}{c}\text { Table 1 } \\
\text { Number, Percentages of Mediation Use } \\
\text { among Medicaid Beneficiaries with Newly-Diagnosed COPD } \\
\text { Medicaid Analytic Extract, 2005-2008 }\end{array}$} \\
\hline & \multicolumn{3}{|c|}{ Any AD Use } & \multicolumn{3}{|c|}{ Any Statin Use } & \multicolumn{3}{|c|}{ Any ICS Use } \\
\hline & $\mathbf{N}$ & Row \% & Sig & $\mathbf{N}$ & Row \% & Sig & $\mathbf{N}$ & Row \% & Sig \\
\hline TOTAL & 7,313 & 47.8 & & 3,720 & 24.3 & & 6,554 & 41.4 & \\
\hline Cohort & & & & & & $*$ & & & \\
\hline $2005-2007$ & 4,148 & 48.0 & & 2,041 & 23.6 & & 3,746 & 43.4 & \\
\hline 2006-2008 & 3,165 & 47.6 & & 1,679 & 25.3 & & 2,808 & 42.2 & \\
\hline Sex & & & $* * *$ & & & $* * *$ & & & $* * *$ \\
\hline Women & 4,853 & 54.6 & & 2,299 & 25.9 & & 4,209 & 47.4 & \\
\hline Men & 2,460 & 38.4 & & 1,421 & 22.2 & & 2,345 & 36.6 & \\
\hline Race/Ethnicity & & & $* * *$ & & & $* * *$ & & & $* * *$ \\
\hline White & 4,121 & 53.9 & & 2,005 & 26.2 & & 3,372 & 44.1 & \\
\hline African American & 1,527 & 37.6 & & 755 & 18.6 & & 1,645 & 40.5 & \\
\hline Others & 1,665 & 46.6 & & 960 & 26.9 & & 1,537 & 43.0 & \\
\hline Age (in years) & & & $* * *$ & & & $* * *$ & & & \\
\hline $40-49$ & 2,787 & 51.9 & & 934 & 17.4 & & 2,262 & 42.1 & \\
\hline $50-59$ & 3,470 & 47.6 & & 1,883 & 25.8 & & 3,129 & 42.9 & \\
\hline $60-64$ & 1,056 & 40.3 & & 903 & 34.4 & & 1,163 & 44.4 & \\
\hline State & & & $* * *$ & & & $* * *$ & & & $* * *$ \\
\hline California & 4,055 & 48.0 & & 2,100 & 24.9 & & 3,481 & 41.2 & \\
\hline Illinois & 1,548 & 48.0 & & 764 & 23.7 & & 1,455 & 45.1 & \\
\hline New York & 1,104 & 51.8 & & 566 & 26.5 & & 1,188 & 55.7 & \\
\hline Texas & 606 & 40.8 & & 290 & 19.5 & & 430 & 29.0 & \\
\hline Income Eligibility & & & $* *$ & & & $* * *$ & & & + \\
\hline Yes & 6,753 & 48.2 & & 3,471 & 24.8 & & 5,976 & 42.7 & \\
\hline No & 560 & 43.7 & & 249 & 19.4 & & 578 & 45.1 & \\
\hline Medical Eligibility & & & & & & $* * *$ & & & \\
\hline Yes & 814 & 48.1 & & 338 & 20.0 & & 740 & 43.7 & \\
\hline No & 6,499 & 47.8 & & 3,382 & 24.9 & & 5,814 & 42.8 & \\
\hline Inflammation-relate & Gultimo & idity & $* * *$ & & & $* * *$ & & & \\
\hline Physical Only & 2,601 & 40.0 & & 2,087 & 32.1 & & 2,787 & 42.8 & \\
\hline Mental Only & 1,011 & 78.8 & & 184 & 14.3 & & 567 & 44.2 & \\
\hline Both & 2,245 & 81.4 & & 846 & 30.7 & & 1,216 & 44.1 & \\
\hline None & 1,456 & 30.7 & & 603 & 12.7 & & 1,984 & 41.9 & \\
\hline
\end{tabular}

(Continued) 


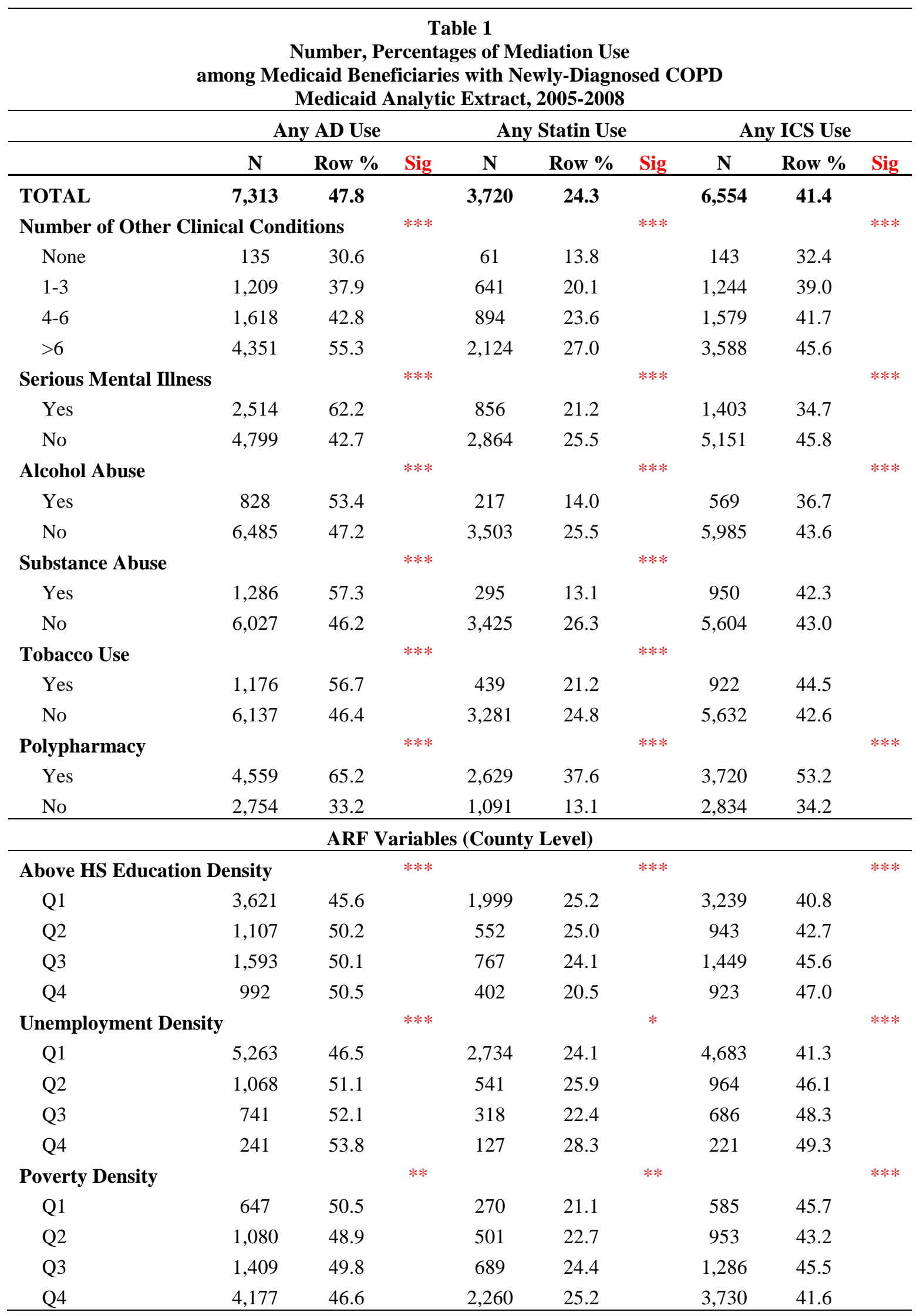




\begin{tabular}{|c|c|c|c|c|c|c|c|c|c|}
\hline \multicolumn{10}{|c|}{$\begin{array}{c}\text { Table 1 } \\
\text { Number, Percentages of Mediation Use } \\
\text { among Medicaid Beneficiaries with Newly-Diagnosed COPD } \\
\text { Medicaid Analytic Extract, 2005-2008 }\end{array}$} \\
\hline & \multicolumn{3}{|c|}{ Any AD Use } & \multicolumn{3}{|c|}{ Any Statin Use } & \multicolumn{3}{|c|}{ Any ICS Use } \\
\hline & $\mathbf{N}$ & Row \% & Sig & $\mathbf{N}$ & Row \% & Sig & $\mathbf{N}$ & Row \% & Sig \\
\hline TOTAL & 7,313 & 47.8 & & 3,720 & 24.3 & & 6,554 & 41.4 & \\
\hline Metro & & & $* * *$ & & & & & & \\
\hline Yes & 6,222 & 47.0 & & 3,202 & 24.2 & & 5,673 & 42.9 & \\
\hline No & 1,091 & 53.3 & & 518 & 25.3 & & 881 & 43.0 & \\
\hline PCP Shortage & & & & & & + & & & \\
\hline Yes & 7,003 & 47.8 & & 3,586 & 24.5 & & 6,288 & 42.9 & \\
\hline No & 310 & 49.5 & & 134 & 21.4 & & 266 & 42.5 & \\
\hline Mental Health Speciali & st Short & & $* * *$ & & & & & & $* * *$ \\
\hline Yes & 6,457 & 47.3 & & 3,313 & 24.3 & & 5,750 & 42.1 & \\
\hline No & 856 & 52.1 & & 407 & 24.8 & & 804 & 48.9 & \\
\hline PCP Density & & & $* * *$ & & & & & & $* * *$ \\
\hline Q1 & 321 & 50.8 & & 161 & 25.5 & & 326 & 51.6 & \\
\hline Q2 & 1,335 & 51.7 & & 674 & 26.1 & & 1,147 & 44.4 & \\
\hline Q3 & 1,136 & 51.8 & & 527 & 24.0 & & 1,025 & 46.7 & \\
\hline Q4 & 4,521 & 45.8 & & 2,358 & 23.9 & & 4,056 & 41.1 & \\
\hline Hospital Beds Density & & & $* * *$ & & & $* *$ & & & $* * *$ \\
\hline Q1 & 432 & 53.9 & & 203 & 25.3 & & 401 & 50.1 & \\
\hline Q2 & 1,230 & 49.2 & & 560 & 22.4 & & 1,092 & 43.6 & \\
\hline Q3 & 3,983 & 46.4 & & 2,168 & 25.2 & & 3,440 & 40.0 & \\
\hline Q4 & 1,668 & 49.2 & & 789 & 23.3 & & 1,621 & 47.8 & \\
\hline Psychiatric Hospital & & & $* * *$ & & & & & & \\
\hline Yes & 4,497 & 46.2 & & 2,395 & 24.6 & & 4,132 & 42.5 & \\
\hline No & 2,816 & 50.7 & & 1,325 & 23.9 & & 2,422 & 43.6 & \\
\hline Pulmonologist Density & & & $* * *$ & & & * & & & $* * *$ \\
\hline High & 4,913 & 45.9 & & 2,546 & 23.8 & & 4,346 & 40.6 & \\
\hline Low & 2,400 & 52.3 & & 1,174 & 25.6 & & 2,208 & 48.1 & \\
\hline Cardiologist Density & & & $* * *$ & & & & & & $* * *$ \\
\hline High & 4,601 & 46.1 & & 2,401 & 24.1 & & 4,094 & 41.0 & \\
\hline Low & 2,712 & 51.1 & & 1,319 & 24.9 & & 2,460 & 46.4 & \\
\hline
\end{tabular}

Note: Based on 15,287 Medicaid Beneficiaries with newly diagnosed COPD and diabetes-free during baseline period obtained from Medicaid Analytic eXtract files observed during 2005-2008. Asterisks represent significant group differences in beneficiary characteristics by medication use obtained from chi-square test.

ICS: Inhaled Corticosteroids; HS: High School PCP: Primary Care Providers; Sig: Significance $* * * \mathrm{p}<.001 ; * * .001 \leq \mathrm{p}<.01 ; * .01 \leq \mathrm{p}<.05$ 
Table 2

Number, Percentage, Unadjusted, Adjusted Odds Ratio and 95\% Confidence Intervals from Logistic Regressions on New-Onset Diabetes among Medicaid Beneficiaries with Newly-Diagnosed COPD Medicaid Analytic Extract, 2005-2008

\begin{tabular}{|c|c|c|c|c|c|c|c|c|}
\hline \multicolumn{9}{|c|}{ New-Onset Diabetes } \\
\hline & $\mathbf{N}$ & Row \% & OR & $95 \% \mathrm{CI}$ & Sig & AOR & $95 \% \mathrm{CI}$ & Sig \\
\hline TOTAL & 967 & 6.3 & & & & & & \\
\hline \multicolumn{9}{|c|}{ Any Antidepressant Use } \\
\hline Yes & 475 & 6.5 & 1.06 & {$[0.93,1.20]$} & & 0.91 & {$[0.78,1.06]$} & \\
\hline No & 492 & 6.2 & & & & & & \\
\hline \multicolumn{9}{|c|}{ Any ICS Use } \\
\hline Yes & 488 & 7.4 & 1.39 & {$[1.22,1.58]$} & $* * *$ & 1.23 & {$[1.07,1.47]$} & $* *$ \\
\hline No & 479 & 5.5 & & & & & & \\
\hline \multicolumn{9}{|c|}{ Any Statin Use } \\
\hline Yes & 344 & 9.2 & 1.79 & {$[1.56,2.05]$} & $* * *$ & 1.48 & {$[1.27,1.72]$} & $* * *$ \\
\hline No & 623 & 5.4 & & & & & & \\
\hline
\end{tabular}

Note: Based on 15,287 Medicaid Beneficiaries with newly diagnosed COPD who were diabetes-free during baseline period obtained from Medicaid Analytic eXtract files observed during 2005-2008. Asterisks represent significant group differences in likelihood of new-onset diabetes by antidepressant, ICS and statin use compared to the reference group (None) obtained from Unadjusted and Adjusted Logistic Regression Analyses.

Adjusted logistic regressions controlled for cohort year, sex, race, age, state, poverty eligibility, inflammationrelated multimorbidity number of other clinical conditions, serious mental illness, alcohol abuse, substance abuse, tobacco use, polypharmacy, county level variables including: above high school education density (quartiles), unemployment density (quartiles), poverty density (quartiles), metro status, primary care shortage area, mental health shortage area, primary care provider density (quartiles), hospital beds density (quartiles), psychiatric hospital, pulmonologist density and cardiologist density in addition to medication use.

ICS: Inhaled Corticosteroids; OR: Odds Ratio; AOR: Adjusted Odds Ratio; CI: Confidence Interval; Sig: Significance

$* * * \mathrm{p}<.001 ; * * .001 \leq \mathrm{p}<.01 ; * .01 \leq \mathrm{p}<.05$ 
Table 3

Number, Percentage, Unadjusted, Adjusted Odds Ratio and 95\% Confidence Intervals

from Logistic Regressions on New-Onset Diabetes

Medicaid Beneficiaries with Newly-Diagnosed COPD

Medicaid Analytic Extract, 2005-2008

\begin{tabular}{lcrcccccc}
\hline \multicolumn{7}{c}{ New-Onset Diabetes } \\
\hline \multicolumn{10}{c}{ NOTAL } & $\mathbf{9 6 7}$ & $\mathbf{6 0 w} \%$ & $\mathbf{O R}$ & $\mathbf{9 5 \%} \mathbf{C I}$ & Sig & AOR & $\mathbf{9 5 \%} \mathbf{C I}$ & Sig \\
\hline Medication Use Categories & & & & & & & & \\
All three & 107 & 10.0 & 2.17 & {$[1.69,2.77]$} & $* * *$ & 1.56 & {$[1.18,2.05]$} & $* *$ \\
AD/Statin & 79 & 7.9 & 1.67 & {$[1.27,2.19]$} & $* * *$ & 1.29 & {$[0.96,1.74]$} \\
AD/ICS & 149 & 6.3 & 1.31 & {$[1.05,1.63]$} & $*$ & 1.09 & {$[0.86,1.39]$} & \\
Statin/ICS & 90 & 12.4 & 2.74 & {$[2.11,3.57]$} & $* * *$ & 2.06 & {$[1.56,2.72]$} & $* * *$ \\
AD only & 140 & 4.9 & 0.99 & {$[0.79,1.24]$} & & 0.90 & {$[0.71,1.14]$} & \\
Statin only & 68 & 7.3 & 1.54 & {$[1.15,2.04]$} & $* *$ & 1.26 & {$[0.94,1.70]$} & \\
ICS only & 142 & 5.9 & 1.22 & {$[0.98,1.53]$} & & 1.11 & {$[0.88,1.39]$} \\
None & 192 & 4.9 & & & & & & \\
\end{tabular}

Note: Based on 15,287 Medicaid Beneficiaries with newly diagnosed COPD who were diabetes-free during baseline period obtained from Medicaid Analytic eXtract files observed during 2005-2008. Asterisks represent significant group differences in likelihood of new-onset diabetes by multiple medication use categories compared to the reference group (None) obtained from Unadjusted and Adjusted Logistic Regression Analyses.

Adjusted logistic regressions controlled for cohort year, sex, race, age, state, poverty eligibility, inflammationrelated multimorbidity number of other clinical conditions, serious mental illness, alcohol abuse, substance abuse, tobacco use, polypharmacy, county level variables including: above high school education density (quartiles), unemployment density (quartiles), poverty density (quartiles), metro status, primary care shortage area, mental health shortage area, primary care provider density (quartiles), hospital beds density (quartiles), psychiatric hospital, pulmonologist density and cardiologist density in addition to medication use.

AD: Antidepressants; ICS: Inhaled corticosteroids OR: Odds Ratio; AOR: Adjusted Odds Ratio; CI: Confidence Interval; Sig: Significance

$* * * \mathrm{p}<.001 ; * * .001 \leq \mathrm{p}<.01 ; * .01 \leq \mathrm{p}<.05$ 
Table 4

Number, Percentage, Unadjusted, Adjusted Odds Ratios and 95\% Confidence Intervals

from Logistic Regressions on New-Onset Diabetes with Type of Statin Use

Medicaid Beneficiaries with Newly-Diagnosed COPD

Medicaid Analytic Extract, 2005-2008

\begin{tabular}{|c|c|c|c|c|c|c|c|c|}
\hline \multicolumn{9}{|c|}{ New-Onset Diabetes } \\
\hline & $\mathbf{N}$ & Row \% & OR & $95 \% \mathrm{CI}$ & Sig & AOR & $95 \% \mathrm{CI}$ & Sig \\
\hline TOTAL & 958 & 6.3 & & & & & & \\
\hline \multicolumn{9}{|c|}{ Type of Statin Use } \\
\hline Lipophilic & 292 & 9.5 & 1.06 & {$[0.76,1.48]$} & & 1.03 & {$[0.73,1.44]$} & \\
\hline Hydrophilic & 43 & 9.0 & & & & & & \\
\hline None & 623 & 5.4 & 0.58 & {$[0.42,0.80]$} & $* * *$ & 0.68 & {$[0.49,0.94]$} & $*$ \\
\hline
\end{tabular}

Note: Based on 15,128 Medicaid Beneficiaries with newly diagnosed COPD who were diabetes-free during baseline period obtained from Medicaid Analytic eXtract files observed during 2005-2008. Asterisks represent significant group differences in likelihood of new-onset diabetes by type of statin use compared to the reference group (None) obtained from Unadjusted and Adjusted Logistic Regression Analyses.

Adjusted logistic regressions controlled for cohort year, sex, race, age, state, poverty eligibility, inflammationrelated multimorbidity number of other clinical conditions, serious mental illness, alcohol abuse, substance abuse, tobacco use, antidepressants use, Inhaled Corticosteroids use, polypharmacy, county level variables including: above high school education density (quartiles), unemployment density (quartiles), poverty density (quartiles), metro status, primary care shortage area, mental health shortage area, primary care provider density (quartiles), hospital beds density (quartiles), psychiatric hospital, pulmonologist density and cardiologist density in addition to medication use.

OR: Odds Ratio; AOR: Adjusted Odds Ratio; CI: Confidence Interval; Sig: Significance

$* * * \mathrm{p}<.001 ; * * .001 \leq \mathrm{p}<.01 ; * .01 \leq \mathrm{p}<.05$ 
Table 5

Number, Percentage, Unadjusted, Adjusted Odds Ratio and 95\% Confidence Intervals

from Logistic Regressions on New-Onset Diabetes

Medicaid Beneficiaries with Newly-Diagnosed COPD

Medicaid Analytic Extract, 2005-2008

\begin{tabular}{lcccccccc}
\hline \multicolumn{10}{c}{ New-Onset Diabetes } \\
\hline & $\mathbf{N}$ & Row \% & OR & $\mathbf{9 5 \%} \mathbf{C I}$ & Sig & AOR & $\mathbf{9 5 \%} \mathbf{C I}$ & Sig \\
\hline TOTAL & $\mathbf{9 6 7}$ & $\mathbf{6 . 3}$ & & & & & & \\
Duration of AD Use & & & & & & & \\
$\quad$ Long-term & 357 & 6.5 & 1.06 & {$[0.92,1.22]$} & 0.91 & {$[0.77,1.07]$} \\
$\quad$ Short-term & 118 & 6.4 & 1.04 & {$[0.84,1.28]$} & 0.92 & {$[0.74,1.14]$} \\
$\quad$ No Use & 492 & 6.2 & & & & & & \\
$\quad$ Duration of Statin Use & & & & & & & & \\
$\quad$ Long-term & 247 & 9.1 & 1.75 & {$[1.50,2.05]$} & $* * *$ & 1.44 & {$[1.22,1.70]$} & $* * *$ \\
$\quad$ Short-term & 97 & 9.7 & 1.89 & {$[1.51,2.36]$} & $* * *$ & 1.58 & {$[1.25,1.99]$} & $* * *$ \\
$\quad$ No Use & 623 & 5.4 & & & & & & \\
& & & & & & & & \\
\end{tabular}

Note: Based on 15,287 Medicaid Beneficiaries with newly diagnosed COPD who were diabetes-free during baseline period obtained from Medicaid Analytic eXtract files observed during 2005-2008. Asterisks represent significant group differences in likelihood of new-onset diabetes by duration of antidepressant and statin use compared to the reference group (None) obtained from Unadjusted and Adjusted Logistic Regression Analyses.

Adjusted logistic regressions controlled for cohort year, sex, race, age, state, poverty eligibility, inflammationrelated multimorbidity number of other clinical conditions, serious mental illness, alcohol abuse, substance abuse, tobacco use, ICS use, polypharmacy, county level variables including: above high school education density (quartiles), unemployment density (quartiles), poverty density (quartiles), metro status, primary care shortage area, mental health shortage area, primary care provider density (quartiles), hospital beds density (quartiles), psychiatric hospital, pulmonologist density and cardiologist density in addition to medication use.

ICS: Inhaled Corticosteroids; OR: Odds Ratio; AOR: Adjusted Odds Ratio; CI: Confidence Interval; Sig: Significance

$$
* * * \mathrm{p}<.001 ; * * .001 \leq \mathrm{p}<.01 ; * .01 \leq \mathrm{p}<.05
$$




\section{CHAPTER 5}




\section{CHAPTER 5: SUMMARY AND CONCLUSIONS}

\section{STUDY SUMMARY}

Chronic Obstructive Pulmonary Disease (COPD), characterized by persistent and progressive airflow limitation caused due to chronic inflammation in the lungs affects more than 15 million Americans in the United States (CDC, 2012; Ford et al., 2013). COPD is being increasingly recognized as a "chronic systemic inflammatory syndrome" due to presence of high levels of systemic and local inflammation. (Barnes \& Celli, 2009) A recent study has established high prevalence of inflammation-related multimorbidity among individuals with COPD. (Schnell et al, 2012) Existing evidence suggests that presence of multimorbidity among individuals with COPD may pose challenges to COPD management (Tsiligianni, Kosmas, Van, \& Tzanakis, 2013). However, no study till now had assessed its impact on COPD management, specifically treatment patterns among individuals with COPD. Furthermore, only a few studies have examined the role of co-existing chronic conditions on COPD-specific outcomes. The findings from these studies point towards negative impact of multimorbidity on COPD-specific outcomes; however, none of the studies until now had systematically evaluated the impact of inflammationrelated multimorbidity on COPD-specific outcomes. This led to the rationale for the Aim1, which examined the association between inflammation-related multimorbidity and COPD treatment patterns and COPD-specific outcomes such as COPD-specific healthcare utilization and healthcare expenditures among individuals with newly-diagnosed COPD. We hypothesized that individuals with newly-diagnosed COPD and inflammation-related multimorbidity will have lower COPD-medication utilization and persistence.

Furthermore, due to the presence of systemic inflammation among patients with COPD, current pharmacologic management is unable to alter the overall prognosis of the disease due to 
their local pulmonary exposure. (M. Cazzola et al., 2007; Vogelmeier \& Wouters, 2011) In this context, some prior studies had examined the beneficial effects of novel anti-inflammatory therapies such as statins in improving lung function, health status and COPD-related exacerbations among those with COPD. (Blamoun et al., 2008; Huang et al., 2011; Ishida et al., 2007; Keddissi et al., 2007; Lawes et al., 2012) None of the studies were generalizable to US population and no study has systematically studied the role of inflammation-related multimorbidity on statin use and COPD-specific outcomes and expenditures. Therefore, the primary objective of Aim 2 was to examine the effectiveness of novel statin therapy in terms of clinical and economic outcomes among individuals with COPD in a real-world practice setting.

Finally, as patients with COPD and inflammation-related multimorbidity are at an increased risk of developing new-onset diabetes (Rana et al., 2004), while examining effectiveness of statin therapies for COPD management, it is important to establish its safety in this population. Moreover, prior studies have suggested that patients with COPD often take multiple medications (Anecchino et al., 2007). These medications may include antidepressants, inhaled corticosteroids (ICS) and statins that have been associated with risk of new-onset diabetes. (Bhattacharjee, Bhattacharya, Kelley, \& Sambamoorthi, 2013; O'Byrne et al., 2012; Sattar et al., 2010; Suissa, Kezouh, \& Ernst, 2010) Thus, the primary objective of Aim 3 was to evaluate the association between antidepressants, ICS and statins and new-onset diabetes among individuals with newly-diagnosed COPD after controlling for comprehensive set of risk factors.

For the purpose of these objectives we used the MAX data for years 2005 through 2008 and conducted retrospective longitudinal analyses 


\section{SUMMARY OF FINDINGS}

Medicaid beneficiaries with newly-diagnosed COPD had very high prevalence of inflammation-related multimorbidity. Presence of inflammation-related multimorbidity was significantly associated with reduced COPD-medication utilization and decreased persistence on long-bronchodilators. Our study findings suggest that COPD medication management may be poor due to competing demands arising from the presence of inflammation-related multimorbidity. Medicaid beneficiaries with inflammation-related multimorbidity were less likely to have COPD-specific utilization compared to those without any inflammation-related multimorbidity. These findings suggest that individuals with inflammation-related multimorbidity may have a greater need for non-COPD related care.

Furthermore, findings from Aim 2 indicate that any statin use was associated with better COPD-specific outcomes compared to no statin use. A closer examination of the data revealed that only those with long-term statin use have better outcomes as compared to those with no statin use. We also found that beneficiaries with inflammation-related multimorbidity and statin use had better COPD-specific outcomes compared to those with multimorbidity and no statin use. These findings suggest that long-term statin use may reduce COPD-specific adverse outcomes. Furthermore, statin use may be particular beneficial for those with inflammationrelated multimorbidity. Our study found that although statin use was associated with the risk of new-onset diabetes, the association was no longer significant in analyses that controlled for selection bias in unobserved characteristics. Thus these findings indicate that the medications may be safe in terms risk of new-onset diabetes. 


\section{STUDY LIMITATIONS}

Our study had some limitations such as limited generalizability due to inclusion of beneficiaries with fee-for-service claims only. The study also used data from only four states which may not provide a full understating of overall Medicaid population. We also excluded dual Medicare-Medicaid enrollees from our population which limits the overall generalizability. Due to unavailability of laboratory data we were not able to control for stage of COPD at diagnosis or confirm the diagnosis of COPD. We only observed prescriptions for specific medications and cannot determine use of these medications. Furthermore, unavailability of laboratory data limited our ability to control for important confounders such as cholesterol levels and baseline blood glucose levels. Additionally we did not have the data for body mass index which is an important predictor of new-onset diabetes.

Despite these limitations, to the best of our knowledge this the first study to extensively examine the relationship between inflammation-related multimorbidity and comprehensive set of COPD-specific outcomes. Therefore, the findings from this study may guide clinical and policy decision making for management of COPD patients with inflammation-related multimorbidity.

\section{UNIQUE CONTRIBUTIONS OF THE STUDY}

Our study aimed to fill the knowledge gap and provide real-world patterns about current pharmacologic management among those with inflammation-related chronic conditions and COPD. As there has been an increasing recognition for organizing care around the individuals rather than the disease (Kadam, 2012) an understanding of how care is delivered among individuals with multimorbidity will have important implications for the overall healthcare management of individuals with COPD. Our study also pioneered in the area of management of 
COPD and provided important evidence that will help in developing clinical practice guidelines for those with COPD and multimorbidity.

Our study contributed to a better understanding of a relationship between inflammationrelated multimorbidity and COPD-specific outcomes. COPD is often understood a disease with local inflammation. Therefore, most pharmacologic medications used in treating COPD focuses on reducing local inflammation and thereby improving only symptoms and not changing the course of disease progression. Recently, investigators have begun to consider COPD as a disease associated with systemic inflammation. By explaining the link between inflammation-related multimorbidity and COPD-specific outcomes, we strengthen the current understanding about inflammation in COPD and provide a novel target area (systemic inflammation) to improve COPD-specific outcomes.

In this context, our study is highly timely and innovative as it examines the association between novel approaches (statin use) for COPD management and COPD-specific outcomes. Another important significance of our study is the use of real-world data that will provide information about individuals with multimorbidity as these individuals are excluded from randomized controlled trials. Our study is also hypothesis generating and will provide the necessary foundation for future research and perhaps randomized clinical trials evaluating the effectiveness and safety of statin therapy among individuals with COPD.

The use of data from the claims of Medicaid beneficiaries enhances the significance of this study. Enrollees in the Medicaid comprise of high-risk vulnerable population that have high rates of COPD and allied multimorbidity. Therefore, proving the real-world evidence about management of COPD and benefits and harms of current and novel therapies among high-risk 
and vulnerable population of Medicaid beneficiaries with COPD will strengthen the evidence in the area of COPD management.

\section{IMPLICATIONS}

Our study highlighted the high prevalence of inflammation-related multimorbidity among non-elderly low-income adults with newly-diagnosed COPD. Our study findings confirm that multimorbidity is common even among the non-elderly and clinical practice and research studies need to examine multimorbidity across the entire life-course (Mercer SW, 2009)

This finding also has implications for healthcare management of these individuals. COPD patients with inflammation-related multimorbidity will have competing demands and require care from multiple providers and specialists such as cardiologists, pulmonologists, mental health specialists, endocrinologists and others. For individuals care needs to be organized around the patient rather than the disease (Kadam, 2012). Furthermore, for individuals with multimorbidity, fee-for-service approach provides little incentive to coordinate care across multiple providers (Chronic Care: Making the Case for Ongoing Care). Financial incentives to physicians for coordinating care are warranted. In this context, Medicaid may want to adopt the Medicare policy initiative of paying physicians who manage care of patients with multimorbidity (DHHS, 2014). Under this new initiative, physicians will be responsible for developing a comprehensive care plan that includes patients' medical, psychological and social needs, monitor medication adherence, and coordinate care across multiple providers (DHHS, 2014)

In this study, we found that beneficiaries who received long-term use of statin medications were less likely to have COPD-specific adverse outcomes compared to no statin use. This finding has implications regarding the long-term adherence to therapies. There is documented evidence of low-rates of long-term adherence to therapies in Medicaid population 
(Kyanko, 2013). Therefore, to realize the benefits of novel therapeutic agents such as statins for management of COPD, surveillance and programs need to be in place for encouraging long-term use of medications.

The current study also evaluated the safety of antidepressants, ICS, and stains with regard to new-onset diabetes. Our study did not find an association between these medications and risk of new-onset diabetes once we controlled for selection bias in unobserved variables. Our study had not included many risk-factors (e.g. obesity, family history, and pre-diabetes). These findings suggest the need to be cautious about making inferences from observational data without rigorous adjustments for selection bias.

Our study utilized Medicaid claims data on non-elderly adults with newly-diagnosed COPD. This has implication for improving access to care through expansion of Medicaid under the affordable care act. In total, 27 states have adopted expanding insurance coverage through Medicaid expansion including California, Illinois and New York (states included in this study). Under this expansion, low-income non-elderly adults with incomes below $133 \%$ of federal poverty level are eligible to be enrolled in Medicaid. Such expansion may include insurance coverage of non-elderly adults over age 40, similar to the population included in this study. As these individuals are likely to have COPD, inflammation related multimorbidity, absolute number of individuals requiring care for these conditions will increase. Therefore, state Medicaid programs will need to specifically examine and solve the issue of provider shortage that may be created due to large influx of individuals with multimorbidity.

\section{DIRECTIONS FOR FUTURE RESEARCH}

Our research utilized retrospective claims data for years through 2005-2008, with rigorous analytical methods to understand the management of COPD among non-elderly adults 
with newly-diagnosed diabetes. Our research contributed to the nascent literature of multimorbidity among individuals with a specific disease namely COPD. We documented the poor COPD management of individuals with inflammation-related multimorbidity and evaluated the benefits and safety of novel therapeutic agents such as statins for COPD management. However, future studies need to go beyond just documenting the existing problems in COPD management. Randomized clinical trials are critical to establish the efficacy, effectiveness, and safety of novel therapeutic agents in management of COPD. Furthermore, future research needs to focus on designing, developing and implementing evidence-based interventions for long-term adherence on medical therapies and improving health outcomes for individuals with COPD and inflammation-related multimorbidity. 


\section{REFERENCES}

Anecchino, C., Rossi, E., Fanizza, C., De Rosa, M., Tognoni, G., \& Romero, M. (2007).

Prevalence of chronic obstructive pulmonary disease and pattern of comorbidities in a general population. International Journal of Chronic Obstructive Pulmonary Disease, 2(4), 567-574.

Barnes, P. J., \& Celli, B. R. (2009). Systemic manifestations and comorbidities of COPD. The European Respiratory Journal, 33(5), 1165-1185. doi:10.1183/09031936.00128008

Bhattacharjee, S., Bhattacharya, R., Kelley, G. A., \& Sambamoorthi, U. (2013). Antidepressant use and new-onset diabetes: A systematic review and meta-analysis. Diabetes/metabolism Research and Reviews, 29(4), 273-284. doi:10.1002/dmrr.2393

Bhattacharya R, Ajmera M, Bhattacharjee S, Sambamoorthi U. Use of antidepressants and statins and short-term risk of new-onset diabetes among high risk adults. Diabetes Res Clin Pract. 2014 Jun 4. pii: S0168-8227(14)00199-5. doi: 10.1016/j.diabres.2014.04.016. [Epub ahead of print]

Blamoun, A. I., Batty, G. N., DeBari, V. A., Rashid, A. O., Sheikh, M., \& Khan, M. A. (2008). Statins may reduce episodes of exacerbation and the requirement for intubation in patients with COPD: Evidence from a retrospective cohort study. International Journal of Clinical Practice, 62(9), 1373-1378. doi:10.1111/j.1742-1241.2008.01731.x

Cazzola, M., Calzetta, L., Bettoncelli, G., Cricelli, C., Romeo, F., Matera, M. G., \& Rogliani, P. (2012). Cardiovascular disease in asthma and COPD: A population-based retrospective cross-sectional study. Respiratory Medicine, 106(2), 249-256. doi:10.1016/j.rmed.2011.07.021; 10.1016/j.rmed.2011.07.021

Cazzola, M., Ciaprini, C., Page, C. P., \& Matera, M. G. (2007). Targeting systemic inflammation: Novel therapies for the treatment of chronic obstructive pulmonary disease. Expert Opinion on Therapeutic Targets, 11(10), 1273-1286.

CDC. (2012). Chronic obstructive pulmonary disease among adults — united states, 2011. (Morbidity and Mortality Weekly Report). Atlanta, GA: Centers for Disease Control and Prevention.

Department of Health and Human Services. (2014) Federal Register; Vol 79. Proposed Rules. http://www.gpo.gov/fdsys/pkg/FR-2014-07-11/pdf/2014-15948.pdf; Accessed Aug 21, 2014 
Ford, E. S., Croft, J. B., Mannino, D. M., Wheaton, A. G., Zhang, X., \& Giles, W. H. (2013). Chronic obstructive pulmonary disease surveillance-united states, 1999-2011. Chest,

Huang, C., Chan, W., Chen, Y., Chen, T., Chou, K., Lin, S., . . Leu, H. (2011). Statin use and hospitalization in patients with chronic obstructive pulmonary disease: A nationwide population-based cohort study in taiwan. Clinical Therapeutics, 33(10), 1365-1370. doi:10.1016/j.clinthera.2011.08.010

Ishida, W., Kajiwara, T., Ishii, M., Fujiwara, F., Taneichi, H., Takebe, N., . . Satoh, J. (2007). Decrease in mortality rate of chronic obstructive pulmonary disease (COPD) with statin use: A population-based analysis in japan. The Tohoku Journal of Experimental Medicine, 212(3), 265-273.

Kadam, U. (2012). Redesigning the general practice consultation to improve care for patients with multimorbidity. BMJ (Clinical Research Ed.), 345, e6202-e6202. doi:10.1136/bmj.e6202

Keddissi, J. I., Younis, W. G., Chbeir, E. A., Daher, N. N., Dernaika, T. A., \& Kinasewitz, G. T. (2007). The use of statins and lung function in current and former smokers. Chest, 132(6), 1764-1771.

Kyanko KA1, Franklin RH, Angell SY. Adherence to chronic disease medications among New York City Medicaid participants. J Urban Health. 2013 Apr;90(2):323-8. doi: 10.1007/s11524-012-9724-4.

Lawes, C. M. M., Thornley, S., Young, R., Hopkins, R., Marshall, R., Chan, W. C., \& Jackson, G. (2012). Statin use in COPD patients is associated with a reduction in mortality: A national cohort study. Primary Care Respiratory Journal: Journal of the General Practice Airways Group, 21(1), 35-40. doi:10.4104/pcrj.2011.00095

O'Byrne, ,P.M., Rennard, S., Gerstein, H., Radner, F., Peterson, S., Lindberg, B., . . Sin, D. D. (2012). Risk of new onset diabetes mellitus in patients with asthma or COPD taking inhaled corticosteroids. Respiratory Medicine, 106(11), 1487-1493. doi:10.1016/j.rmed.2012.07.011

Rana, J. S., Mittleman, M. A., Sheikh, J., Hu, F. B., Manson, J. E., Colditz, G. A., . . Camargo,Carlos A.,,Jr. (2004). Chronic obstructive pulmonary disease, asthma, and risk of type 2 diabetes in women. Diabetes Care, 27(10), 2478-2484.

Sattar, N., Preiss, D., Murray, H. M., Welsh, P., Buckley, B. M., de Craen, A.,J.M., . . Ford, I. (2010). Statins and risk of incident diabetes: A collaborative meta-analysis of randomised statin trials. Lancet, 375(9716), 735-742. doi:10.1016/S0140-6736(09)61965-6 
Schnell, K., Weiss, C. O., Lee, T., Krishnan, J. A., Leff, B., Wolff, J. L., \& Boyd, C. (2012). The prevalence of clinically-relevant comorbid conditions in patients with physician-diagnosed COPD: A cross-sectional study using data from NHANES 1999-2008. BMC Pulmonary Medicine, 12, 26-26. doi:10.1186/1471-2466-12-26

Suissa, S., Kezouh, A., \& Ernst, P. (2010). Inhaled corticosteroids and the risks of diabetes onset and progression. The American Journal of Medicine, 123(11), 1001-1006. doi:10.1016/j.amjmed.2010.06.019

Tsiligianni, I. G., Kosmas, E., Van, d. M., \& Tzanakis, N. (2013). Managing comorbidity in COPD: A difficult task. Current Drug Targets, 14(2), 158-176. 